Bull. Soc. math. France

134 (1), 2006, p. 33-82

\title{
CASCADE OF PHASES IN TURBULENT FLOWS
}

\author{
BY Christophe Cheverry
}

\begin{abstract}
This article is devoted to incompressible Euler equations (or to NavierStokes equations in the vanishing viscosity limit). It describes the propagation of quasi-singularities. The underlying phenomena are consistent with the notion of a cascade of energy.

RÉSumÉ (Cascade de phases pour des fluides turbulents). - Cet article étudie les équations d'Euler incompressible (ou de Navier-Stokes en présence de viscosité évanescente). On y décrit la propagation de quasi-singularités. Les phénomènes sous-jacents confirment l'idée selon laquelle il se produit une cascade d'énergie.
\end{abstract}

\section{Introduction}

Consider incompressible fluid equations

$$
\partial_{t} \boldsymbol{u}+(\boldsymbol{u} \cdot \nabla) \boldsymbol{u}+\nabla \boldsymbol{p}=0, \quad \operatorname{div} \boldsymbol{u}=0, \quad(t, x) \in[0, T] \times \mathbb{R}^{d},
$$

where $\boldsymbol{u}={ }^{t}\left(\boldsymbol{u}^{1}, \ldots, \boldsymbol{u}^{d}\right) \in \mathbb{R}^{d}$ is the fluid velocity and $\boldsymbol{p} \in \mathbb{R}$ is the pressure. The structure of weak solutions of $(\mathcal{E})$ in $d$-space dimensions with $d \geq 2$ is a problem of wide current interest [3], [5], [25]. The questions are how to describe

Texte reçu le 24 février 2004, révisé le 27 août 2004, accepté le 1er octobre 2004. Christophe Cheverry, IRMAR, Université de Rennes I, Campus de Beaulieu, 35042 Rennes Cedex (France) • E-mail : christophe.cheverry@univ-rennes1.fr 2000 Mathematics Subject Classification. - 35A21, 35C20, 35L, 35Q30, 35Q35.

Key words and phrases. — Fluid mechanics, Euler and Navier-Stokes equations, asymptotic expansions, nonlinear geometric optics, propagation of singularities, closure problems, turbulence. 
the phenomena with adequate models and how to visualize the results in spite of their complexity. We will achieve a small step in these two directions.

According to the physical intuition, the appearance of singularities is linked with the increase of the vorticity. Along this line, we have to mark the contributions [2] and [10]. Interesting objects are solutions which do not blow up in finite time but whose associated vorticities increase arbitrarily fast. These are quasi-singularities. Their study is of practical importance.

Typical examples of quasi-singularities are oscillations. This is a well-known fact going back to [4], [26]. The works [4] and [26] rely on phenomenological considerations and engineering experiments. Further developments are related to homogenization [14], [15], compensated compactness [12], [18] and non linear geometric optics [7], [8], [9].

DiPerna and Majda [12] show the persistence of oscillations in three dimensional Euler equations $(d=3)$. To this end, they select parameters $\varepsilon \in] 0,1]$ and look at

$$
\boldsymbol{u}_{s}^{\varepsilon}(t, x):={ }^{t}\left(\boldsymbol{g}\left(x_{2}, \varepsilon^{-1} x_{2}\right), 0, \boldsymbol{h}\left(x_{1}-\boldsymbol{g}\left(x_{2}, \varepsilon^{-1} x_{2}\right) t, x_{2}, \varepsilon^{-1} x_{2}\right)\right)
$$

where $\boldsymbol{g}\left(x_{2}, \theta\right)$ and $\boldsymbol{h}\left(x_{1}, x_{2}, \theta\right)$ are smooth bounded functions with period 1 in $\theta$. They remark that the functions $\boldsymbol{u}_{s}^{\varepsilon}$ are exact smooth solutions of $(\mathcal{E})$ and they let $\varepsilon$ goes to zero. Yet, this construction is of a very special form. First, it comes from shear layers (these are steady $2 \mathrm{D}$ solutions) as

$$
\widetilde{\boldsymbol{u}}_{s}^{\varepsilon}(t, x)=\widetilde{\boldsymbol{u}}_{s}^{\varepsilon}(0, x)={ }^{t}\left(\boldsymbol{g}\left(x_{2}, \varepsilon^{-1} x_{2}\right), 0\right) \in \mathbb{R}^{2} .
$$

Secondly, it involves a phase $\varphi_{0}(t, x) \equiv x_{2}$ which does not depend on $\varepsilon$. Of course, this is a common fact [11], [21], [20], [28] when dealing with such large amplitude high frequency waves. Nevertheless, this is far from giving a complete idea of what can happen. Our aim in this paper is to develop a theory which allows to remove the two restrictions mentioned above.

Section 2 is devoted to notations.

Section 3 gives the main results.

Subsection 3.1 states Theorem 3.1. Introduce the geometrical phase

$$
\varphi_{g}^{\varepsilon}(t, x):=\varphi_{0}(t, x)+\sum_{k=1}^{\ell-1} \varepsilon^{k / \ell} \varphi_{k}(t, x), \quad \ell \in \mathbb{N}_{*} .
$$

Fix $b=(\ell, N) \in \mathbb{N}^{2}$ where the integers $\ell$ and $N$ are such that $0<\ell<N$. Theorem 3.1 provides with approximate solutions $\boldsymbol{u}_{\mathrm{b}}^{\varepsilon}$ defined on the interval $[0, T]$ with $T>0$ and having the form

$$
\begin{aligned}
\boldsymbol{u}_{b}^{\varepsilon}(t, x) & ={ }^{t}\left(\boldsymbol{u}_{b}^{\varepsilon 1}, \ldots, \boldsymbol{u}_{b}^{\varepsilon d}\right)(t, x) \\
& =\boldsymbol{u}_{0}(t, x)+\sum_{k=1}^{N} \varepsilon^{k / \ell} U_{k}\left(t, x, \varepsilon^{-1} \varphi_{g}^{\varepsilon}(t, x)\right)
\end{aligned}
$$

TOME $134-2006-\mathrm{N}^{\mathrm{O}} 1$ 
where the smooth profiles

$$
U_{k}(t, x, \theta)={ }^{t}\left(U_{k}^{1}, \ldots, U_{k}^{d}\right)(t, x, \theta) \in \mathbb{R}^{d}, \quad 1 \leq k \leq N
$$

are periodic functions of $\theta \in \mathbb{T}:=\mathbb{R} / \mathbb{Z}$. We assume that

$$
\exists(t, x, \theta) \in[0, T] \times \mathbb{R}^{d} \times \mathbb{T}, \quad \partial_{\theta} U_{1}(t, x, \theta) \neq 0 .
$$

The family $\left\{\boldsymbol{u}_{b}^{\varepsilon}\right\}_{\varepsilon \in] 0,1]}$ is $\varepsilon$-stratified [20] with respect to the phase $\varphi_{g}^{\varepsilon}$ with in general $\varphi_{g}^{\varepsilon} \not \equiv \varphi_{0}$. The presence in $\varphi_{g}^{\varepsilon}$ of the non trivial functions $\varphi_{k}$ with $1 \leq k \leq \ell-1$ is necessary and sufficient to encompass all the geometrical features of the propagation.

We say that $\left\{\boldsymbol{u}_{b}^{\varepsilon}\right\}_{\varepsilon}$ is a weak, a strong or a turbulent oscillation according as we have respectively $\ell=1, \ell=2$ or $\ell \geq 3$. The order of magnitude of the energy of the oscillations is $\varepsilon^{1 / \ell}$. Compute the vorticities associated with $\boldsymbol{u}_{b}^{\varepsilon}$. These are the skew-symmetric matrices $\Omega_{b}^{\varepsilon}=\left(\Omega_{b j}^{\varepsilon i}\right)_{1 \leq i, j \leq d}$ where

$$
\begin{aligned}
\Omega_{b j}^{\varepsilon i}(t, x):= & \left(\partial_{j} \boldsymbol{u}_{b}^{\varepsilon i}-\partial_{i} \boldsymbol{u}_{b}^{\varepsilon j}\right)(t, x) \\
= & \sum_{k=1}^{N} \varepsilon^{k / \ell-1}\left(\partial_{j} \varphi_{g}^{\varepsilon} \partial_{\theta} U_{k}^{i}-\partial_{i} \varphi_{g}^{\varepsilon} \partial_{\theta} U_{k}^{j}\right)\left(t, x, \varepsilon^{-1} \varphi_{g}^{\varepsilon}(t, x)\right) \\
& +\left(\partial_{j} \boldsymbol{u}_{0}^{i}-\partial_{i} \boldsymbol{u}_{0}^{j}\right)(t, x)+\sum_{k=1}^{N} \varepsilon^{k / \ell}\left(\partial_{j} U_{k}^{i}-\partial_{i} U_{k}^{j}\right)\left(t, x, \varepsilon^{-1} \varphi_{g}^{\varepsilon}(t, x)\right) .
\end{aligned}
$$

The principal term in $\Omega_{b}^{\varepsilon}$ is of size $\varepsilon^{1 / \ell-1}$. When $\ell \geq 2$, there is no uniform majoration in $L^{p}$ on the family $\left\{\Omega_{b}^{\varepsilon}\right\}_{\varepsilon \in] 0,1]}$ since

$$
\lim _{\varepsilon \rightarrow 0}\left\|\Omega_{b}^{\varepsilon}\right\|_{L^{p}\left([0, T] \times \mathbb{R}^{d}\right)}=+\infty, \quad \forall p \in[1, \infty] .
$$

In particular, if $d=3$, there is no uniform control on the enstrophy

$$
\int_{0}^{T} \int_{\mathbb{R}^{3}}\left|\omega_{b}^{\varepsilon}(t, x)\right|^{2} \mathrm{~d} t \mathrm{~d} x, \quad \omega_{b}^{\varepsilon}(t, x):=\left(\nabla \wedge \boldsymbol{u}_{b}^{\varepsilon}\right)(t, x) \equiv \Omega_{b}^{\varepsilon}(t, x) .
$$

We see here that strong and turbulent oscillations are examples of quasisingularities. Observe that the expansion (1.2) involves a more complicated structure than in (1.1) though the corresponding regime is less singular.

Subsection 3.2 states the Proposition 3.1. Introduce the complete phase

$$
\varphi_{b}^{\varepsilon}(t, x):=\varphi_{0}(t, x)+\sum_{k=1}^{N} \varepsilon^{k / \ell} \varphi_{k}(t, x) .
$$

Proposition 3.1 deals with approximate solutions $\widetilde{\boldsymbol{u}}_{b}^{\varepsilon}$ defined on the interval $[0, T]$ with $T>0$ and having the form

$$
\begin{aligned}
\widetilde{\boldsymbol{u}}_{b}^{\varepsilon}(t, x) & ={ }^{t}\left(\widetilde{\boldsymbol{u}}_{b}^{\varepsilon 1}, \ldots, \widetilde{\boldsymbol{u}}_{b}^{\varepsilon d}\right)(t, x) \\
& =\boldsymbol{u}_{0}(t, x)+\sum_{k=1}^{N} \varepsilon^{k / \ell} \widetilde{U}_{k}\left(t, x, \varepsilon^{-1} \varphi_{b}^{\varepsilon}(t, x)\right)
\end{aligned}
$$

BULLETIN DE LA SOCIÉTÉ MATHÉMATIQUe DE FRANCE 
where the smooth profiles

$$
\widetilde{U}_{k}(t, x, \theta)={ }^{t}\left(\widetilde{U}_{k}^{1}, \ldots, \widetilde{U}_{k}^{d}\right)(t, x, \theta) \in \mathbb{R}^{d}, \quad 1 \leq k \leq N
$$

are periodic functions of $\theta \in \mathbb{T}$. Again

$$
\exists(t, x, \theta) \in[0, T] \times \mathbb{R}^{d} \times \mathbb{T}, \quad \partial_{\theta} \widetilde{U}_{1}(t, x, \theta) \neq 0 .
$$

Section 4 shows at first Proposition 3.1 and then Theorem 3.1.

The proof of Proposition 3.1 is based on some induction argument which is quite straightforward. In fact, the difficulty is hidden in the introduction of the adjusting phase

$$
\varphi_{a}^{\varepsilon}(t, x):=\varepsilon^{-1}\left(\varphi_{b}^{\varepsilon}-\varphi_{g}^{\varepsilon}\right)(t, x)=\sum_{k=l}^{N} \varepsilon^{k / \ell-1} \varphi_{k}(t, x) .
$$

Indeed, the use of the geometrical phase $\varphi_{g}^{\varepsilon}$ does not suffice to perform the BKW analysis. Among other things, the extra terms $\varphi_{k}$ with $\ell \leq k \leq N$ must be incorporated in order to put the system of formal equations in a triangular form.

Subsection 4.2 explains how to deduce Theorem 3.1 from Proposition 3.1. It mainly consists in eliminating the adjusting phase (and in checking that the remainder created by that operation is small) as well as in replacing the small divergence of Proposition 3.1 by a zero divergence.

Section 5 interprets the results 3.1 .

It starts with various comments related to the Leray projector, the infinite accuracy of approximate solutions, the finite speed of propagation and the large time existence.

Subsection 5.2 proceeds to a careful study of the hierarchy of phases. We examine successively the phase shift $\varphi_{1}$, the phase shift $\varphi_{2}$, and the other terms $\varphi_{k}$ with $3 \leq k \leq N$.

The formal construction reveals that the phase shift $\varphi_{1}$ and the terms $\varphi_{k}$ with $2 \leq k \leq \ell-1$ play different parts. The rôle of $\varphi_{1}$ is partly revealed in the articles [7] and [8] which deal with the case $\ell=2$. When $\ell \geq 3$, the phenomenon to emphasize is the creation of the $\varphi_{k}$ with $2 \leq k \leq \ell-1$. Indeed, suppose that

$$
\varphi_{2}(0, .) \equiv \cdots \equiv \varphi_{\ell-1}(0, .) \equiv 0, \quad \ell \geq 3 .
$$

Then, generically, we find

$$
\exists t \in] 0, T], \quad \varphi_{2}(t, .) \not \equiv 0, \ldots, \varphi_{\ell-1}(t, .) \not \equiv 0 .
$$

Now, starting with large amplitude waves (this corresponds to the limit case $\ell=+\infty)$ that is

$$
\boldsymbol{u}_{\infty}^{\varepsilon}(0, x)=\sum_{k=0}^{\infty} \varepsilon^{k} U_{k}\left(0, x, \varepsilon^{-1} \varphi_{0}(0, x)\right), \quad \partial_{\theta} U_{0} \not \equiv 0,
$$

TOME $134-2006-\mathrm{N}^{\mathrm{O}} 1$ 
the description of $\boldsymbol{u}_{\infty}^{\varepsilon}(t,$.$) on the interval [0, T]$ with $T>0$ needs the introduction of an infinite cascade of phases $\varphi_{k}$. The scenario is the following. Oscillations of the velocity develop spontaneously in all the intermediate frequencies $\varepsilon^{k / \ell-1}$ and in all the corresponding directions $\nabla \varphi_{k}(t, x)$. This expresses turbulent features in the flow.

Subsection 5.3 alludes to closure problems. This is the classical difficulty encountered when dealing with expansions as $\boldsymbol{u}_{b}^{\varepsilon}$. It is solved here through the introduction of the $\varphi_{k}$ with $1 \leq k \leq N$.

Subsection 5.4 insists on obvious instabilities which are mechanisms of amplification which can be detected just by looking at the BKW analysis presented before. It allows to retrieve known non linear instability results on Euler equations (see Proposition 5.1).

Subsection 5.5 and subsection 5.6 are mainly heuristical. They could also interest researchers in Fluid mechanics. They contain no precise statement or proof but consist in reading Theorem 3.1 in the light of previous numerical, mathematical or physical results. They derive many informations about microstructures, compensated compactness and non linear geometric optics. They also confirm observations which have been made in the statistical approach of turbulences [16], [24].

Section 6 consider parabolic perturbations of Euler equations. This change of framework has two main motivations.

First, it has a physical meaning. Most real models involve some viscosity. And, even if it were only at a formal level, it is interesting to determine what is the size and the structure of the dissipation terms which could be incorporated without changing the phenomena under study.

Secondly, it has implications on the stability. The expressions $\boldsymbol{u}_{b}^{\varepsilon}$ are only approximate solutions of Euler equations, yielding small error terms $\boldsymbol{f}_{b}^{\varepsilon}$ as source terms. The matter is to know if the addition of (well-adjusted) dissipation terms implies the existence of exact solutions (of Navier-Stokes type equations) which coincide with $\boldsymbol{u}_{b}^{\varepsilon}(0,$.$) at time t=0$, which are defined on $[0, T]$ where $T>0$ is independent on $\varepsilon$, and which are close to approximate divergence free solutions like $\boldsymbol{u}_{b}^{\varepsilon}$.

These two directions are difficult tasks. In this paper, we will be satisfied to touch on these subjects.

In Subsection 6.1, we build (Proposition 6.1) approximate solutions $\left\{u_{b}^{\varepsilon}\right\}_{\varepsilon}$ to some Navier-Stokes equation $(\mathcal{N S})$ involving the variables $t, x$ and $\theta$. We start by describing the properties of the parabolic perturbation. The viscosity is vanishing and anisotropic. It could be real or artificial but it must be compatible with the complete phase $\varphi_{b}^{\varepsilon}$. Then, we adapt the proof of subsection 4.1 to this new setting. In particular, we are faced with the study of the divergence free relation written in the $(t, x, \theta)$ variables. 
In Subsection 6.2, we look at the stability of strong oscillations $\left\{u_{(2, N)}^{\varepsilon}\right\}_{\varepsilon}$ given by Proposition 6.1. Theorem 6.1 shows that exact solutions $u^{\varepsilon}$ of $(\mathcal{N S})$ exist on some interval $[0, T]$ where $T>0$ is independent on the parameter $\varepsilon \in] 0,1]$. Moreover, the family $\left\{u^{\varepsilon}\right\}_{\varepsilon}$ remains close to $\left\{u_{(2, N)}^{\varepsilon}\right\}_{\varepsilon}$.

\section{Notations}

Variables. - Let $T \in \mathbb{R}^{+}$. The time variable is $t \in[0, T]$. Let $d \in \mathbb{N} \backslash\{0,1\}$. The space variables are $(x, \theta) \in \mathbb{R}^{d} \times \mathbb{T}$ where $x \in \mathbb{R}^{d}$ is the slow variable whereas $\theta \in \mathbb{T}:=\mathbb{R} / \mathbb{Z}$ is the fast (periodic) variable. Mark the ball

$$
B(0, R]:=\left\{x \in \mathbb{R}^{d} ;|x|^{2}:=\sum_{i=1}^{d} x_{i}^{2} \leq R\right\}, \quad R \in \mathbb{R}^{+} .
$$

The state variables are the velocity field $u={ }^{t}\left(u^{1}, \cdots, u^{d}\right) \in \mathbb{R}^{d}$ and the pressure $p \in \mathbb{R}$. Given $(u, \widetilde{u}) \in\left(\mathbb{R}^{d}\right)^{2}$, define

$$
u \cdot \widetilde{u}:=\sum_{i=1}^{d} u^{i} \widetilde{u}^{i}, \quad|u|^{2}:=u \cdot u, \quad u \otimes \widetilde{u}:=\left(u^{j} \widetilde{u}^{i}\right)_{1 \leq i, j \leq d} .
$$

The symbol $S^{d}$ is for the set of symmetric quadratic forms on $\mathbb{R}^{d}$. An element $\mathfrak{q} \in S^{d}$ can be represented by some $d \times d$ matrix $\left(\mathfrak{q}^{i j}\right)_{1 \leq i, j \leq d}$ and

$$
\mathfrak{q}(\xi, \widetilde{\xi})=\sum_{1 \leq i, j \leq d} \mathfrak{q}^{i j} \xi_{i} \xi_{j}=\mathfrak{q}(\widetilde{\xi}, \xi), \quad \forall(\xi, \widetilde{\xi}) \in\left(\mathbb{R}^{d}\right)^{2} .
$$

Functional spaces. - Distinguish the expressions $\boldsymbol{u}(t, x)$ which do not depend on the variable $\theta$ from the expressions $u(t, x, \theta)$ which depend on $\theta$. The boldfaced type $\boldsymbol{u}$ is used in the first case whereas the letter $u$ is employed in the second situation.

Note $C_{b}^{\infty}\left([0, T] \times \mathbb{R}^{d}\right)$ the space of functions in $[0, T] \times \mathbb{R}^{d}$ with bounded continuous derivatives of any order.

Let $m \in \mathbb{N}$. The Sobolev space $H^{m}$ is the set of functions

$$
u(x, \theta)=\sum_{k \in \mathbb{Z}} \boldsymbol{u}_{k}(x) \mathrm{e}^{i k \theta}
$$

such that

$$
\|u\|_{H^{m}}^{2}:=\sum_{k \in \mathbb{Z}}\left(1+|k|^{2}\right)^{m} \int_{\mathbb{R}^{d}}\left(1+|\xi|^{2}\right)^{m}\left|\widehat{\boldsymbol{u}}_{k}(\xi)\right|^{2} \mathrm{~d} \xi<\infty
$$

where

$$
\mathcal{F}(\boldsymbol{u})(\xi)=\widehat{\boldsymbol{u}}(\xi):=(2 \pi)^{-\frac{1}{2} d} \int_{\mathbb{R}^{d}} \mathrm{e}^{-i x \cdot \xi} \boldsymbol{u}(x) \mathrm{d} x, \quad \xi \in \mathbb{R}^{d} .
$$

TOME $134-2006-\mathrm{N}^{\mathrm{O}} 1$ 
With these conventions, the condition $\boldsymbol{u} \in H^{m}$ means simply that

$$
\|\boldsymbol{u}\|_{H^{m}}^{2}:=\int_{\mathbb{R}^{d}}\left(1+|\xi|^{2}\right)^{m}|\widehat{\boldsymbol{u}}(\xi)|^{2} \mathrm{~d} \xi<\infty .
$$

Define the Sobolev spaces

$$
H_{T}^{m}:=\left\{u ; \partial_{t}^{j} u \in L^{2}\left([0, T] ; H^{m-j}\right), \forall j \in\{0, \ldots, m\}\right\}
$$

and the Banach spaces

$$
\mathcal{W}_{T}^{m}:=\left\{u ; u \in C^{j}\left([0, T] ; H^{m-j}\right), \forall j \in\{0, \ldots, m\}\right\}
$$

with the corresponding norms

$$
\|u\|_{H_{T}^{m}}^{2}:=\sum_{j=0}^{m} \int_{0}^{T}\left\|\partial_{t}^{j} u(t, .)\right\|_{H^{m}}^{2} \mathrm{~d} t, \quad\|u\|_{\mathcal{W}_{T}^{m}}:=\sup _{t \in[0, T]} \sum_{j=0}^{m}\left\|\partial_{t}^{j} u(t, .)\right\|_{H^{m}} .
$$

Consider also

$$
\begin{aligned}
H_{\infty}^{m} & :=\bigcap_{T \in \mathbb{R}^{+}} H_{T}^{m}, \quad H_{T}^{\infty}:=\bigcap_{m \in \mathbb{N}} H_{T}^{m}, \quad H_{\infty}^{\infty}:=\bigcap_{T \in \mathbb{R}} H_{T}^{\infty}, \mathcal{W}_{\infty}^{m}:=\bigcap_{T \in \mathbb{R}^{+}} \mathcal{W}_{T}^{m}, \\
\mathcal{W}_{T}^{\infty} & :=\bigcap_{m \in \mathbb{N}} \mathcal{W}_{T}^{m}, \quad \mathcal{W}_{\infty}^{\infty}:=\bigcap_{T \in \mathbb{R}} \mathcal{W}_{T}^{\infty} .
\end{aligned}
$$

When $m=0$, replace $H^{0}$ with $L^{2}$. Any function $u \in L^{2}$ can be decomposed according to

$$
u(t, x, \theta)=\langle u\rangle(t, x)+u^{*}(t, x, \theta)=\bar{u}(t, x)+u^{*}(t, x, \theta)
$$

where $\langle u\rangle$ or $\bar{u}$ are the mean values

$$
\langle u\rangle(t, x)=\bar{u}(t, x):=\int_{\mathbb{T}} u(t, x, \theta) \mathrm{d} \theta .
$$

Let $\Gamma$ be the symbol of any of the spaces $H^{m}, H_{T}^{m}, \mathcal{W}_{T}^{m}, \ldots$ defined before. In order to specify the functions with mean value zero, introduce

$$
\Gamma^{*}:=\{u \in \Gamma ; \bar{u} \equiv 0\} .
$$

Mark also

$$
\operatorname{supp}_{x} u^{*}:=\text { closure of }\left\{x \in \mathbb{R}^{d} ;\left\|u^{*}(x, .)\right\|_{L^{2}(\mathbb{T})} \neq 0\right\} .
$$

Differential operators. - Note

$$
\begin{gathered}
\partial_{t} \equiv \partial_{0}:=\frac{\partial}{\partial t}, \quad \partial_{\theta} \equiv \partial_{d+1}:=\frac{\partial}{\partial \theta}, \quad \partial_{j}:=\frac{\partial}{\partial x_{j}}, j \in\{1, \ldots, d\}, \\
\nabla:=\left(\partial_{1}, \ldots, \partial_{d}\right), \quad \Delta:=\Delta_{x}+\partial_{\theta}^{2}=\partial_{1}^{2}+\cdots+\partial_{d}^{2}+\partial_{\theta}^{2} .
\end{gathered}
$$

BULLETIN DE LA SOCIÉTÉ MATHÉMATIQUE DE FRANCE 
Let $u \in \mathcal{W}_{T}^{\infty}$. Define

$$
\begin{aligned}
u \cdot \nabla & :=u^{1} \partial_{1}+\cdots+u^{d} \partial_{d}, \\
\operatorname{div} u & :=\partial_{1} u^{1}+\cdots+\partial_{d} u^{d} \in \mathbb{R}, \\
\operatorname{div}(u \otimes \widetilde{u}) & :=\sum_{j=1}^{d}{ }^{t}\left(\partial_{j}\left(u^{j} \widetilde{u}^{1}\right), \ldots, \partial_{j}\left(u^{j} \widetilde{u}^{d}\right)\right) \in \mathbb{R}^{d} .
\end{aligned}
$$

Employ the bracket $\langle., .\rangle_{H}$ for the scalar product in the Hilbert space $H$. Note $\mathcal{L}(E ; F)$ the space of linear continuous applications $T: E \rightarrow F$ where $E$ and $F$ are Banach spaces. The symbol $\mathcal{L}(E)$ is simply for $\mathcal{L}(E ; E)$. Introduce the commutator

$$
[A ; B]:=A \circ B-B \circ A, \quad(A, B) \in \mathcal{L}(E)^{2} .
$$

Let $r \in \mathbb{Z}$. The operator $T$ is in $\mathfrak{L}^{r}$ if

$$
\|T\|_{\mathcal{L}\left(H_{T}^{m+r} ; H_{T}^{m}\right)}<\infty, \quad \forall m \in \mathbb{N} .
$$

Let $\varepsilon_{0}>0$. The family of operators $\left\{T^{\varepsilon}\right\}_{\varepsilon}$ is in $\mathfrak{U} \mathfrak{L}^{r}$ if

$$
\sup _{\left.\varepsilon \in] 0, \varepsilon_{0}\right]}\left\|T^{\varepsilon}\right\|_{\mathcal{L}\left(H_{T}^{m+r} ; H_{T}^{m}\right)}<\infty, \quad \forall m \in \mathbb{N} .
$$

Given a family $\left\{f^{\varepsilon}\right\}_{\varepsilon}$ such that $f^{\varepsilon}(t, x, \theta) \in \mathcal{W}_{T}^{\infty}$ for all $\left.\left.\varepsilon \in\right] 0, \varepsilon_{0}\right]$, we say that $\left\{f^{\varepsilon}\right\}_{\varepsilon}=O\left(\varepsilon^{r}\right)$ if

$$
\sup _{\left.\varepsilon \in] 0, \varepsilon_{0}\right]} \varepsilon^{-r}\left\|f^{\varepsilon}\right\|_{\mathcal{W}_{T}^{m}}<\infty, \quad \forall m \in \mathbb{N} .
$$

Given a family $\left\{\boldsymbol{f}^{\varepsilon}\right\}_{\varepsilon}$ such that $\boldsymbol{f}^{\varepsilon}(t, x) \in \mathcal{W}_{T}^{\infty}$ for all $\left.\left.\varepsilon \in\right] 0, \varepsilon_{0}\right]$, we say that $\left\{\boldsymbol{f}^{\varepsilon}\right\}_{\varepsilon}=O\left(\varepsilon^{r}\right)$ if

$$
\sup _{\left.\varepsilon \in] 0, \varepsilon_{0}\right]} \varepsilon^{-r+m}\left\|\boldsymbol{f}^{\varepsilon}\right\|_{\mathcal{W}_{T}^{m}}<\infty, \quad \forall m \in \mathbb{N} .
$$

Observe that the two preceding definitions have very different significations according as we use the letter $f$ or the boldfaced type $f$. In particular, the second inequalities correspond to $\varepsilon$-stratified estimates. The families $\left\{f^{\varepsilon}\right\}_{\varepsilon}$ or $\left\{\boldsymbol{f}^{\varepsilon}\right\}_{\varepsilon}$ are $O\left(\varepsilon^{\infty}\right)$ if they are $O\left(\varepsilon^{r}\right)$ for all $r \in \mathbb{Z}$.

\section{Statement of the results}

The description of incompressible flows in turbulent regime is a delicate question. No systematic analysis is yet available. However, special approximate solutions with rapidly varying structure in space and time can be exhibited. Their construction is summarized in this chapter 3 .

TOME $134-2006-\mathrm{N}^{\mathrm{O}} 1$ 
3.1. The main theorem. - Select smooth functions

$$
\boldsymbol{u}_{00} \in H^{\infty}, \quad \varphi_{00} \in C^{1}\left(\mathbb{R}^{d}\right), \quad \nabla \varphi_{00} \in C_{b}^{\infty}\left(\mathbb{R}^{d}\right) .
$$

Impose the non degeneracy hypothesis

$$
\exists c>0 ; \quad\left|\nabla \varphi_{00}(x)\right| \geq 4 c, \quad \forall x \in \mathbb{R}^{d} .
$$

For $T>0$ small enough, the equation $(\mathcal{E})$ associated with

$$
\boldsymbol{u}_{0}(0, x)=\boldsymbol{u}_{00}(x), \quad \forall x \in \mathbb{R}^{d}
$$

has a smooth solution $\boldsymbol{u}_{0}(t, x) \in \mathcal{W}_{T}^{\infty}$. It satisfies

$$
\partial_{t} \boldsymbol{u}_{0}+\left(\boldsymbol{u}_{0} \cdot \nabla\right) \boldsymbol{u}_{0}+\nabla \boldsymbol{p}_{0}=0, \quad \operatorname{div} \boldsymbol{u}_{0}=0, \quad(t, x) \in[0, T] \times \mathbb{R}^{d} .
$$

Solve the eiconal equation

$$
\partial_{t} \varphi_{0}+\left(\boldsymbol{u}_{0} \cdot \nabla\right) \varphi_{0}=0, \quad(t, x) \in[0, T] \times \mathbb{R}^{d}
$$

with the initial data

$$
\varphi_{0}(0, x)=\varphi_{00}(x), \quad \forall x \in \mathbb{R}^{d} .
$$

If necessary, restrict the time $T$ in order to have

$$
\left|\nabla \varphi_{0}(t, x)\right| \geq 2 c, \quad \forall(t, x) \in[0, T] \times \mathbb{R}^{d} .
$$

Call $\Pi_{0}(t, x)$ the orthogonal projector from $\mathbb{R}^{d}$ onto the hyperplane

$$
\nabla \varphi_{0}(t, x)^{\perp}:=\left\{u \in \mathbb{R}^{d} ; u \cdot \nabla \varphi_{0}(t, x)=0\right\} .
$$

In other words

$$
\Pi_{0}(t, x) u=u-\left|\nabla \varphi_{0}(t, x)\right|^{-2}\left(u \cdot \nabla \varphi_{0}(t, x)\right) \nabla \varphi_{0}(t, x), \quad \forall u \in \mathbb{R}^{d} .
$$

One can construct approximate solutions to the incompressible Euler equation $(\mathcal{E})$ as the superposition of the exact solution $\left(\boldsymbol{u}_{0}, \boldsymbol{p}_{0}\right)$ and an arbitrarily large number of profiles, like in (1.2).

Theorem 3.1. - Choose any $b=(\ell, N) \in \mathbb{N}^{2}$ such that $0<\ell\left(3+\frac{1}{2} d\right)<N$. Select arbitrary data

$$
\bar{U}_{k 0}(x) \in H^{\infty}, 1 \leq k \leq N-\ell ; \quad \varphi_{k 0}(x) \in H^{\infty}, 1 \leq k \leq \ell-1 .
$$

Select any data

$$
U_{k 0}^{*}(x, \theta) \in H^{\infty}, \quad\left\langle U_{k 0}^{*}\right\rangle \equiv 0, \quad 1 \leq k \leq N-\ell
$$

satisfying the polarization conditions

$$
U_{k 0}^{*}(x, \theta)=\Pi_{0}(0, x) U_{k 0}^{*}(x, \theta), \quad \forall(x, \theta) \in \mathbb{R}^{d} \times \mathbb{T} .
$$

Suppose moreover that the functions $U_{k 0}(\cdot, \theta)$ for all $k \in\{1, \ldots, N-\ell\}$ and for all $\theta \in \mathbb{T}$ have a support which is contained in a fixed compact set $D \subset \mathbb{R}^{d}$. The following preliminaries (i), (ii) and (iii) can be achieved. 
(i) There are finite sequences $\left\{U_{k}\right\}_{1 \leq k \leq N}$ and $\left\{P_{k}\right\}_{1 \leq k \leq N}$ with

$$
U_{k}(t, x, \theta) \in \mathcal{W}_{T}^{\infty}, \quad P_{k}(t, x, \theta) \in \mathcal{W}_{T}^{\infty}, \quad 1 \leq k \leq N,
$$

and which are such that

$$
\begin{gathered}
\Pi_{0}(0, x) U_{k}^{*}(0, x, \theta)=U_{k 0}^{*}(x, \theta), \quad \forall k \in\{1, \ldots, N-\ell\}, \\
\bar{U}_{k}(0, x)=\bar{U}_{k 0}(x), \quad \forall k \in\{1, \ldots, N-\ell\} .
\end{gathered}
$$

(ii) There is a finite sequence $\left\{\varphi_{k}\right\}_{1 \leq k \leq \ell-1}$ with

$$
\varphi_{k}(t, x) \in \mathcal{W}_{T}^{\infty}, \quad \forall k \in\{1, \ldots, \ell-1\},
$$

and which is such that

$$
\varphi_{k}(0, x)=\varphi_{k 0}(x), \quad \forall k \in\{1, \ldots, \ell-1\} .
$$

(iii) There is $\left.\left.\varepsilon_{0} \in\right] 0,1\right]$ and there are correctors

$$
\left.\left.\boldsymbol{c} \boldsymbol{u}_{b}^{\varepsilon}(t, x) \in \mathcal{W}_{T}^{\infty}, \quad \boldsymbol{c p}_{b}^{\varepsilon}(t, x) \in \mathcal{W}_{T}^{\infty}, \quad \varepsilon \in\right] 0, \varepsilon_{0}\right],
$$

which give rise to families satisfying

$$
\left\{\boldsymbol{c} \boldsymbol{u}_{b}^{\varepsilon}\right\}_{\varepsilon}=O\left(\varepsilon^{N / \ell-2-\frac{1}{2} d}\right), \quad\left\{\boldsymbol{c p}_{b}^{\varepsilon}\right\}_{\varepsilon}=O\left(\varepsilon^{(N+1) / \ell}\right) .
$$

With the materials of (i), (ii) and (iii), construct the geometrical phase

$$
\varphi_{g}^{\varepsilon}(t, x):=\varphi_{0}(t, x)+\sum_{k=1}^{\ell-1} \varepsilon^{k / \ell} \varphi_{k}(t, x)
$$

the initial data

$$
\boldsymbol{h}_{b}^{\varepsilon}(x):=\boldsymbol{u}_{00}(x)+\sum_{k=1}^{N} \varepsilon^{k / \ell} U_{k}\left(0, x, \varepsilon^{-1} \varphi_{g}^{\varepsilon}(0, x)\right)+\boldsymbol{c} \boldsymbol{u}_{b}^{\varepsilon}(0, x),
$$

and the functions $\boldsymbol{u}_{b}^{\varepsilon}$ and $\boldsymbol{p}_{b}^{\varepsilon}$ defined according to

$$
\left\{\begin{array}{l}
\boldsymbol{u}_{b}^{\varepsilon}(t, x):=\boldsymbol{u}_{0}(t, x)+\sum_{k=1}^{N} \varepsilon^{k / \ell} U_{k}\left(t, x, \varepsilon^{-1} \varphi_{g}^{\varepsilon}(t, x)\right)+\boldsymbol{c} \boldsymbol{u}_{b}^{\varepsilon}(t, x), \\
\boldsymbol{p}_{b}^{\varepsilon}(t, x):=\boldsymbol{p}_{0}(t, x)+\sum_{k=1}^{N} \varepsilon^{k / \ell} P_{k}\left(t, x, \varepsilon^{-1} \varphi_{g}^{\varepsilon}(t, x)\right)+\boldsymbol{c p}_{b}^{\varepsilon}(t, x) .
\end{array}\right.
$$

Then, all these expressions can be adjusted so that the functions $\boldsymbol{u}_{b}^{\varepsilon}$ and $\boldsymbol{p}_{b}^{\varepsilon}$ satisfy on the interval $[0, T]$ the incompressible Euler equation $(\mathcal{E})$ up to some small forcing term $\boldsymbol{f}_{b}^{\varepsilon}$. More precisely

$$
\partial_{t} \boldsymbol{u}_{b}^{\varepsilon}+\left(\boldsymbol{u}_{b}^{\varepsilon} \cdot \nabla\right) \boldsymbol{u}_{b}^{\varepsilon}+\nabla \boldsymbol{p}_{b}^{\varepsilon}=\boldsymbol{f}_{b}^{\varepsilon}, \quad \operatorname{div} \boldsymbol{u}_{b}^{\varepsilon}=0, \quad \boldsymbol{u}_{b}^{\varepsilon}(0, x)=\boldsymbol{h}_{b}^{\varepsilon}(x)
$$

and we have $\left\{\boldsymbol{f}_{b}^{\varepsilon}\right\}_{\varepsilon}=O\left(\varepsilon^{N / \ell-3-\frac{1}{2} d}\right)$.

In practice, Theorem 3.1 should be applied with $N / \ell \gg 1$ and with a main profile $U_{10}^{*}$ adjusted so that

$$
\exists(x, \theta) \in \mathbb{R}^{d} \times \mathbb{T} ; \quad U_{10}^{*}(x, \theta) \neq 0 .
$$

TOME $134-2006-\mathrm{N}^{\mathrm{O}} 1$ 
It follows that $U_{1}^{*} \not \equiv 0$ and the principal oscillating part of the approximate solution $\boldsymbol{u}_{b}^{\varepsilon}$ is given by

$$
\varepsilon^{1 / \ell} U_{1}^{*}\left(t, x, \varepsilon^{-1} \varphi_{g}^{\varepsilon}(t, x)\right), \quad \ell \in \mathbb{N}_{*} .
$$

Observe also that the approximate solution can be written

$$
\begin{aligned}
\boldsymbol{u}_{b}^{\varepsilon}(t, x)=\boldsymbol{u}_{0} & (t, x)+\boldsymbol{c} \boldsymbol{u}_{b}^{\varepsilon}(t, x) \\
& +\varepsilon^{1 / \ell} U^{\varepsilon}\left(t, x, \varepsilon^{-1} \varphi_{0}(t, x), \varepsilon^{1 / \ell-1} \varphi_{1}(t, x), \ldots, \varepsilon^{-1 / \ell} \varphi_{\ell-1}(t, x)\right)
\end{aligned}
$$

where the profile $U^{\varepsilon}\left(t, x, \theta_{0}, \theta_{1}, \ldots, \theta_{\ell-1}\right) \in C^{\infty}\left([0, T] \times \mathbb{R}^{d} \times \mathbb{T}^{d}\right)$ is defined according to

$$
U^{\varepsilon}\left(t, x, \theta_{0}, \theta_{1}, \ldots, \theta_{\ell-1}\right):=\sum_{k=1}^{N} \varepsilon^{(k-1) / \ell} U_{k}\left(t, x, \theta_{0}+\theta_{1}+\cdots+\theta_{\ell-1}\right) .
$$

According to this interpretation, Theorem 3.1 is a multiphase non linear geometric optics result. However, since all the terms $\varphi_{k}$ are grouped together inside the geometrical phase $\varphi_{g}^{\varepsilon}$, it must be rather understood as an extension of some monophase analysis.

Theorem 3.1 will be obtained as the consequence of the result which is exposed in the next Subsection 3.2.

3.2. The key of the analysis. - The core of the analysis is the proof of the following result.

Proposition 3.1. - Choose any $b=(\ell, N) \in \mathbb{N}^{2}$ such that $0<\ell<N$. Select arbitrary data

$$
\left\langle\widetilde{U}_{k 0}\right\rangle(x) \in H^{\infty}, \quad 1 \leq k \leq N-\ell, \quad \varphi_{k 0}(x) \in H^{\infty}, \quad 1 \leq k \leq N .
$$

Select any data

$$
\widetilde{U}_{k 0}^{*}(x, \theta) \in H^{\infty}, \quad\left\langle\widetilde{U}_{k 0}^{*}\right\rangle \equiv 0, \quad 1 \leq k \leq N-\ell
$$

satisfying the polarization conditions

$$
\widetilde{U}_{k 0}^{*}(x, \theta)=\Pi_{0}(0, x) \widetilde{U}_{k 0}^{*}(x, \theta), \quad \forall(x, \theta) \in \mathbb{R}^{d} \times \mathbb{T} .
$$

The following preliminaries (i) and (ii) can be achieved.

(i) There are finite sequences $\left\{\widetilde{U}_{k}\right\}_{1 \leq k \leq N}$ and $\left\{\widetilde{P}_{k}\right\}_{1 \leq k \leq N}$ with

$$
\widetilde{U}_{k}(t, x, \theta) \in \mathcal{W}_{T}^{\infty}, \quad \widetilde{P}_{k}(t, x, \theta) \in \mathcal{W}_{T}^{\infty}, \quad 1 \leq k \leq N,
$$

and which are such that

$$
\begin{gathered}
\Pi_{0}(0, x) \widetilde{U}_{k}^{*}(0, x, \theta)=\widetilde{U}_{k 0}^{*}(x, \theta), \quad \forall k \in\{1, \ldots, N-\ell\}, \\
\left\langle\widetilde{U}_{k}\right\rangle(0, x)=\left\langle\widetilde{U}_{k 0}\right\rangle(x), \quad \forall k \in\{1, \ldots, N-\ell\} .
\end{gathered}
$$

BULLETIN DE LA SOCIÉtÉ MATHÉMATIQUE DE FRANCE 
(ii) There is a finite sequence $\left\{\varphi_{k}\right\}_{1 \leq k \leq N}$ with

$$
\varphi_{k}(t, x) \in \mathcal{W}_{T}^{\infty}, \quad \forall k \in\{1, \ldots, N\}
$$

and which is such that

$$
\varphi_{k}(0, x)=\varphi_{k 0}(x), \quad \forall k \in\{1, \ldots, N\} .
$$

With the materials of (i) and (ii), construct the complete phase

the initial data

$$
\varphi_{b}^{\varepsilon}(t, x):=\varphi_{0}(t, x)+\sum_{k=1}^{N} \varepsilon^{k / \ell} \varphi_{k}(t, x),
$$

$$
\widetilde{h}_{b}^{\varepsilon}(x, \theta):=\boldsymbol{u}_{00}(x)+\sum_{k=1}^{N} \varepsilon^{k / \ell} \widetilde{U}_{k}(0, x, \theta),
$$

and the functions $\widetilde{u}_{b}^{\varepsilon}$ and $\widetilde{p}_{b}^{\varepsilon}$ defined according to

$$
\left\{\begin{array}{l}
\widetilde{u}_{b}^{\varepsilon}(t, x, \theta)=\boldsymbol{u}_{0}(t, x)+\sum_{k=1}^{N} \varepsilon^{k / \ell} \widetilde{U}_{k}(t, x, \theta), \\
\widetilde{p}_{b}^{\varepsilon}(t, x, \theta)=\boldsymbol{p}_{0}(t, x)+\sum_{k=1}^{N} \varepsilon^{k / \ell} \widetilde{P}_{k}(t, x, \theta) .
\end{array}\right.
$$

Then, all these expressions can be adjusted so that the functions $\widetilde{u}_{b}^{\varepsilon}$ and $\widetilde{p}_{b}^{\varepsilon}$ satisfy on the domain $[0, T] \times \mathbb{R}^{d} \times \mathbb{T}$ the following singular system

$$
\left\{\begin{aligned}
& \partial_{t} \widetilde{u}_{b}^{\varepsilon}+\left(\widetilde{u}_{b}^{\varepsilon} \cdot \nabla\right) \widetilde{u}_{b}^{\varepsilon}+\nabla \widetilde{p}_{b}^{\varepsilon} \\
&+\varepsilon^{-1}\left(\partial_{t} \varphi_{b}^{\varepsilon}+\widetilde{u}_{b}^{\varepsilon} \cdot \nabla \varphi_{b}^{\varepsilon}\right) \partial_{\theta} \widetilde{u}_{b}^{\varepsilon}+\varepsilon^{-1} \partial_{\theta} \widetilde{p}_{b}^{\varepsilon} \nabla \varphi_{b}^{\varepsilon}=\widetilde{f}_{b}^{\varepsilon}, \\
& \operatorname{div} \widetilde{u}_{b}^{\varepsilon}+\varepsilon^{-1} \nabla \varphi_{b}^{\varepsilon} \cdot \partial_{\theta} \widetilde{u}_{b}^{\varepsilon}=\widetilde{g}_{b}^{\varepsilon}, \widetilde{u}_{b}^{\varepsilon}(0, x, \theta)=\widetilde{h}_{b}^{\varepsilon}(x, \theta),
\end{aligned}\right.
$$

where the correctors $\tilde{f}_{b}^{\varepsilon}$ and $\widetilde{g}_{b}^{\varepsilon}$ are such that

$$
\left.\left.\tilde{f}_{b}^{\varepsilon}(t, x, \theta) \in \mathcal{W}_{T}^{\infty}, \quad \widetilde{g}_{b}^{\varepsilon}(t, x, \theta) \in \mathcal{W}_{T}^{\infty}, \quad \forall \varepsilon \in\right] 0, \varepsilon_{0}\right],
$$

and satisfy

$$
\left\{\tilde{f}_{b}^{\varepsilon}\right\}_{\varepsilon}=O\left(\varepsilon^{(N+1-\ell) / \ell}\right), \quad\left\{\widetilde{g}_{b}^{\varepsilon}\right\}_{\varepsilon}=O\left(\varepsilon^{(N+1-\ell) / \ell}\right) .
$$

Proposition 3.1 works with the variables $(t, x, \theta)$. Of course, the given informations can be interpreted in the variables $(t, x)$. Define

$$
\left\{\begin{array}{l}
\widetilde{\boldsymbol{u}}_{b}^{\varepsilon}(t, x):=\boldsymbol{u}_{0}(t, x)+\sum_{k=1}^{N} \varepsilon^{k / \ell} \widetilde{U}_{k}\left(t, x, \varepsilon^{-1} \varphi_{b}^{\varepsilon}(t, x)\right), \\
\widetilde{\boldsymbol{p}}_{b}^{\varepsilon}(t, x):=\boldsymbol{p}_{0}(t, x)+\sum_{k=1}^{N} \varepsilon^{k / \ell} \widetilde{P}_{k}\left(t, x, \varepsilon^{-1} \varphi_{b}^{\varepsilon}(t, x)\right), \\
\widetilde{\boldsymbol{h}}_{b}^{\varepsilon}(t, x):=\widetilde{h}_{b}^{\varepsilon}\left(t, x, \varepsilon^{-1} \varphi_{b}^{\varepsilon}(t, x)\right) \\
\widetilde{\boldsymbol{f}}_{b}^{\varepsilon}(t, x):=\widetilde{f}_{b}^{\varepsilon}\left(t, x, \varepsilon^{-1} \varphi_{b}^{\varepsilon}(t, x)\right) \\
\widetilde{\boldsymbol{g}}_{b}^{\varepsilon}(t, x):=\widetilde{g}_{b}^{\varepsilon}\left(t, x, \varepsilon^{-1} \varphi_{b}^{\varepsilon}(t, x)\right) .
\end{array}\right.
$$

TOME $134-2006-\mathrm{N}^{\mathrm{O}} 1$ 
Then, deduce from (3.10) that

$$
\partial_{t} \widetilde{\boldsymbol{u}}_{b}^{\varepsilon}+\left(\widetilde{\boldsymbol{u}}_{b}^{\varepsilon} \cdot \nabla\right) \widetilde{\boldsymbol{u}}_{b}^{\varepsilon}+\nabla \widetilde{\boldsymbol{p}}_{b}^{\varepsilon}=\tilde{\boldsymbol{f}}_{b}^{\varepsilon}, \quad \operatorname{div} \widetilde{\boldsymbol{u}}_{b}^{\varepsilon}=\widetilde{\boldsymbol{g}}_{b}^{\varepsilon}, \quad \widetilde{\boldsymbol{u}}_{b}^{\varepsilon}(0, x)=\widetilde{\boldsymbol{h}}_{b}^{\varepsilon}(x) .
$$

The information (3.11) yields

$$
\left\{\tilde{\boldsymbol{f}}_{b}^{\varepsilon}\right\}_{\varepsilon}=O\left(\varepsilon^{(N+1-\ell) / \ell}\right), \quad\left\{\widetilde{\boldsymbol{g}}_{b}^{\varepsilon}\right\}_{\varepsilon}=O\left(\varepsilon^{(N+1-\ell) / \ell}\right) .
$$

There are two main differences between Theorem 3.1 and Proposition 3.1:

- First, the function $\widetilde{\boldsymbol{u}}_{b}^{\varepsilon}$ is not divergence free. Something must be done to pass from (3.13) to (3.7).

- Secondly, the expressions $\widetilde{\boldsymbol{u}}_{b}^{\varepsilon}$ and $\widetilde{\boldsymbol{p}}_{b}^{\varepsilon}$ involve the complete phase $\varphi_{b}^{\varepsilon}(t, x)$ instead of the geometrical phase $\varphi_{g}^{\varepsilon}(t, x)$. Indeed, we have incorporated the additional terms $\varphi_{k}$ with $\ell \leq k \leq N$.

Remark that

$$
\varphi_{b}^{\varepsilon}(t, x)=\varphi_{g}^{\varepsilon}(t, x)+\varepsilon \varphi_{a}^{\varepsilon}(t, x), \quad \varphi_{a}^{\varepsilon}(t, x):=\sum_{k=l}^{N} \varepsilon^{k / \ell-1} \varphi_{k}(t, x),
$$

where $\varphi_{a}^{\varepsilon}(t, x)$ is the adjusting phase.

\section{Proofs of the results}

Theorem 3.1 follows from Proposition 3.1. Therefore, to understand Theorem 3.1, it is necessary to go through the proof of Proposition 3.1.

4.1. Proof of Proposition 3.1. - We work in the framework of Proposition 3.1. For convenience, we drop in this paragraph 4.1 the tilde ' $\sim$, on the expressions $U_{k 0}, U_{k}, P_{k}, h_{\mathrm{b}}^{\varepsilon}, u_{\mathrm{b}}^{\varepsilon}, p_{\mathrm{b}}^{\varepsilon}, f_{\mathrm{b}}^{\varepsilon}$ and $g_{\mathrm{b}}^{\varepsilon}$. These modifications concern only this proof. We hope that it will not induce confusions.

The phase $\varphi_{b}^{\varepsilon}$ will be determined during the process. For a while, do as if it is some already known data. Because of (3.5), for $\varepsilon$ small enough, the function $\varphi_{\mathrm{b}}^{\varepsilon}$ is not stationary

$$
\left.\left.\exists \varepsilon_{0}>0 ; \quad\left|\nabla \varphi_{b}^{\varepsilon}(t, x)\right| \geq c>0, \quad \forall(\varepsilon, t, x) \in\right] 0, \varepsilon_{0}\right] \times[0, T] \times \mathbb{R}^{d} .
$$

Therefore, we can define the application $\Pi_{b}^{\varepsilon}(t, x)$ which is the orthogonal projector on the hyperplane $\nabla \varphi_{b}^{\varepsilon}(t, x)^{\perp} \subset \mathbb{R}^{d}$. In other words

$$
\Pi_{b}^{\varepsilon}(t, x) u=u-\left|\nabla \varphi_{b}^{\varepsilon}(t, x)\right|^{-2}\left(u \cdot \nabla \varphi_{b}^{\varepsilon}(t, x)\right) \nabla \varphi_{b}^{\varepsilon}(t, x) .
$$

Adopt the conventions

$$
\begin{gathered}
X_{b}^{\varepsilon}(t, x):=\nabla \varphi_{b}^{\varepsilon}(t, x)=\sum_{k=0}^{N} \varepsilon^{k / \ell} X_{k}(t, x), \quad X_{k}(t, x):=\nabla \varphi_{k}(t, x), \\
\Pi_{b}^{\varepsilon}(t, x)=\sum_{k=0}^{\infty} \varepsilon^{k / \ell} \Pi_{k}(t, x), \quad \Pi_{k}(t, x) \in \mathcal{W}_{T}^{\infty} .
\end{gathered}
$$

BULletin DE LA SOCIÉtÉ MATHÉMATIQUE DE FRANCE 
Simple computations indicate that the access to $\Pi_{k}$ needs only the knowledge of the $X_{j}$ for $j \leq k$. Introduce

$$
\begin{gathered}
v_{b}^{\varepsilon}:=X_{b}^{\varepsilon} \cdot u_{b}^{\varepsilon}=\sum_{k=0}^{\infty} \varepsilon^{k / \ell} V_{k}, \quad V_{k}=X_{k} \cdot \boldsymbol{u}_{0}+\sum_{j=0}^{k-1} X_{j} \cdot U_{k-j}, \\
w_{b}^{\varepsilon}:=\Pi_{b}^{\varepsilon} u_{b}^{\varepsilon}=\sum_{k=0}^{\infty} \varepsilon^{k / \ell} W_{k}, \quad W_{k}=\Pi_{k} \boldsymbol{u}_{0}+\sum_{j=0}^{k-1} \Pi_{j} U_{k-j} .
\end{gathered}
$$

By construction

$$
u_{b}^{\varepsilon}=v_{b}^{\varepsilon}\left|X_{b}^{\varepsilon}\right|^{-2} X_{b}^{\varepsilon}+w_{b}^{\varepsilon} .
$$

It follows that

$$
U_{k}=V_{k}\left|X_{0}\right|^{-2} X_{0}+W_{k}+\mathcal{H}_{k},
$$

where $\mathcal{H}_{k}$ depends only on the $X_{j}$ for $j \leq k$ and on the $U_{j}$ for $j \leq k-1$.

The conditions prescribed in Proposition 3.1 on the initial data $U_{k}^{*}(0, x, \theta)$ allow to fix the functions $\nabla \varphi_{0}(0, x) \cdot U_{k}^{*}(0, x, \theta)$ as we want. Since

$$
V_{k}^{*}=\nabla \varphi_{0} \cdot U_{k}^{*}+\sum_{j=1}^{k-1} X_{j} \cdot U_{k-j}^{*}
$$

the same is true (by induction) for the components $V_{k}^{*}(0, x, \theta)$. To begin with, impose the polarization conditions

$$
P_{k}^{*} \equiv V_{k}^{*} \equiv 0, \quad \forall k \in\{1, \cdots, \ell\} .
$$

Adjust a priori the geometrical phase $\varphi_{g}^{\varepsilon}$ so that

$$
\partial_{t} \varphi_{k}+\bar{V}_{k}=0, \quad \forall k \in\{1, \ldots, \ell-1\}
$$

which implies the approximate eiconal equation

$$
\partial_{t} \varphi_{g}^{\varepsilon}+\left(\bar{u}_{b}^{\varepsilon} \cdot \nabla\right) \varphi_{g}^{\varepsilon}=\sum_{k=l}^{\infty} \varepsilon^{k / \ell} \bar{V}_{k}=O(\varepsilon) .
$$

To simplify notations, we will sometimes drop the indices $\varepsilon$ and $b$ at the level of $u_{b}^{\varepsilon}, v_{b}^{\varepsilon}, w_{b}^{\varepsilon}, p_{b}^{\varepsilon}, \Pi_{b}^{\varepsilon}$ and $\varphi_{b}^{\varepsilon}$. The interpretation of $(\mathcal{E})$ in the variables $(t, x, \theta)$ leads to the singular system

$$
\left\{\begin{array}{l}
\partial_{t} u+(u \cdot \nabla) u+\nabla p+\varepsilon^{-1}\left(\partial_{t} \varphi+v\right) \partial_{\theta} u+\varepsilon^{-1} \partial_{\theta} p \nabla \varphi=0 \\
\operatorname{div} u+\varepsilon^{-1} \partial_{\theta} v=0
\end{array}\right.
$$

The scalar component $v$ is subjected to

$$
\begin{aligned}
\partial_{t} v+(u \cdot \nabla) & +X \cdot \nabla p+\varepsilon^{-1}\left(\partial_{t} \varphi+v\right) \partial_{\theta} v \\
& +\varepsilon^{-1} \partial_{\theta} p\|X\|^{2}-\left(\partial_{t} X+(u \cdot \nabla) X\right) \cdot u=0
\end{aligned}
$$

TOME $134-2006-\mathrm{N}^{\mathrm{O}} 1$ 
The vector valued function $w$ satisfies

$$
\partial_{t} w+(u \cdot \nabla) w+\Pi \nabla p+\varepsilon^{-1}\left(\partial_{t} \varphi+v\right) \partial_{\theta} w-\left(\partial_{t} \Pi+(u \cdot \nabla) \Pi\right) u=0 .
$$

Substitute the expressions $u_{b}^{\varepsilon}$ and $p_{b}^{\varepsilon}$ given by (3.9) into (4.5). Then arrange the terms according to the different powers of $\varepsilon$ which are in factor.

The contributions coming from the orders $\varepsilon^{1 / \ell-1}, \ldots, \varepsilon^{-1 / \ell}$ and $\varepsilon^{0}$ are eliminated through (4.3), (4.4) and the constraints imposed on $\left(\boldsymbol{u}_{0}, \boldsymbol{p}_{0}\right)$.

Now, look at the terms in front of $\varepsilon^{j / \ell}$ with $j \in \mathbb{N}_{*}$. It remains

$$
\left\{\begin{aligned}
\partial_{t} U_{j}+\sum_{k=0}^{j}\left(U_{k} \cdot \nabla\right) U_{j-k} & +\nabla P_{j}+\sum_{k=0}^{j} \partial_{\theta} P_{\ell+k} \nabla \varphi_{j-k} \\
& +\sum_{k=0}^{j-1}\left(\partial_{t} \varphi_{\ell+k}+V_{\ell+k}\right) \partial_{\theta} U_{j-k}=0 \\
\operatorname{div} U_{j}+\partial_{\theta} V_{j+\ell}=0 . &
\end{aligned}\right.
$$

Proceed in a similar manner with (4.6). It gives

$$
\begin{aligned}
& \partial_{t} V_{j}+\sum_{k=0}^{j}\left(U_{k} \cdot \nabla\right) V_{j-k}+\sum_{k=0}^{j-1}\left(\partial_{t} \varphi_{\ell+k}+V_{\ell+k}\right) \partial_{\theta} V_{j-k} \\
& \quad+\sum_{k=0}^{j} X_{k} \cdot \nabla P_{j-k}-\sum_{k=0}^{j} \partial_{t} X_{k} \cdot U_{j-k}-\sum_{k=0}^{j}\left(\sum_{\ell=0}^{k}\left(U_{k-\ell} \cdot \nabla\right) X_{\ell}\right) \cdot U_{j-k} \\
& +\sum_{k=1}^{j-1}\left(\sum_{\ell=0}^{k} X_{\ell} \cdot X_{k-\ell}\right) \partial_{\theta} P_{j+\ell-k}+\left|X_{0}\right|^{2} \partial_{\theta} P_{j+\ell}=0
\end{aligned}
$$

The same operation with (4.7) yields

$$
\begin{gathered}
\partial_{t} W_{j}+\sum_{k=0}^{j}\left(U_{k} \cdot \nabla\right) W_{j-k}+\sum_{k=0}^{j-1}\left(\partial_{t} \varphi_{\ell+k}+V_{\ell+k}\right) \partial_{\theta} W_{j-k} \\
+\sum_{k=0}^{j} \Pi_{k} \nabla P_{j-k}-\sum_{k=0}^{j} \partial_{t} \Pi_{k} U_{j-k}-\sum_{k=0}^{j}\left(\sum_{\ell=0}^{k}\left(U_{k-\ell} \cdot \nabla\right) \Pi_{\ell}\right) U_{j-k}=0 .
\end{gathered}
$$

Then extract the mean value of (4.8)

$$
\left\{\begin{aligned}
\partial_{t} \bar{U}_{j}+\left(\boldsymbol{u}_{0} \cdot \nabla\right) \bar{U}_{j}+\left(\bar{U}_{j} \cdot \nabla\right) \boldsymbol{u}_{0}+\nabla \bar{P}_{j} \\
\quad+\sum_{k=1}^{j-1}\left\langle\left(U_{k} \cdot \nabla\right) U_{j-k}\right\rangle+\sum_{k=1}^{j-1}\left\langle V_{\ell+k}^{*} \partial_{\theta} U_{j-k}\right\rangle=0 \\
\operatorname{div} \bar{U}_{j}=0 .
\end{aligned}\right.
$$

BULLETIN DE LA SOCIÉTÉ MATHÉMATIQUE DE FRANCE 
Adopt the conventions

$$
\begin{aligned}
& U_{k} \equiv 0, \quad \forall k \in\{1-\ell, \ldots,-1\} \\
& \partial_{\theta}^{-1} U(\theta):=\int_{0}^{\theta} U(s) d s-\int_{\mathbb{T}}\left\{\int_{0}^{\theta} U(s) \mathrm{d} s\right\} \mathrm{d} \theta .
\end{aligned}
$$

Observe that the divergence free relation furnishes

$$
V_{j+\ell}^{*}=-\operatorname{div} \partial_{\theta}^{-1} U_{j}^{*}, \quad \forall j \in \mathbb{N}_{*} .
$$

Consider the inductive reasoning based on the following hypothesis $\left(H_{j}\right)$ where $j \geq 1$ :

HYPOTHESIS $\left(H_{j}\right)$

(i) The expressions $U_{1}, \ldots, U_{j}$ and $P_{1}, \ldots, P_{j}$ are known.

(ii) The phases $\varphi_{1}, \ldots, \varphi_{j}$ are identified. The same is true for the vectors $X_{1}, \ldots, X_{j}$ and the projectors $\Pi_{1}, \ldots, \Pi_{j}$. Moreover, the following relations are satisfied

$$
\partial_{t} \varphi_{k}+\bar{V}_{k}=0, \quad \forall k \in\{1, \ldots, j\} .
$$

(iii) The correctors $V_{j+1}^{*}, \ldots, V_{j+\ell}^{*}$ and $P_{j+1}^{*}, \ldots, P_{j+\ell}^{*}$ are identified. In particular, the scalars $V_{j+1}^{*}, \ldots, V_{j+\ell}^{*}$ are given by the relations

$$
V_{k}^{*}=-\operatorname{div} \partial_{\theta}^{-1} U_{k-\ell}^{*}, \quad \forall k \in\{1, \ldots, j+\ell\} .
$$

Verification of $\left(H_{1}\right)$. - Note first that the conditions which are obtained in (4.14) when $j=1$ and $k \in\{1, \ldots, \ell-1\}$ are compatible with the previous restrictions in (4.3). Indeed, the preceding conventions on the profiles $U_{k}$ with $k \in\{1-\ell, \ldots,-1\}$ have been adjusted accordingly.

The mean value $\bar{U}_{1}$ is obtained by solving

$$
\left\{\begin{array}{l}
\partial_{t} \bar{U}_{1}+\left(\boldsymbol{u}_{0} \cdot \nabla\right) \bar{U}_{1}+\left(\bar{U}_{1} \cdot \nabla\right) \boldsymbol{u}_{0}+\nabla \bar{P}_{1}=0 \\
\operatorname{div} \bar{U}_{1}=0, \quad \bar{U}_{1}(0, x)=\bar{U}_{10}(x) .
\end{array}\right.
$$

Extract

$$
\bar{V}_{1}=X_{1} \cdot \boldsymbol{u}_{0}+X_{0} \cdot \bar{U}_{1}, \quad \bar{W}_{1}=\Pi_{1} \boldsymbol{u}_{0}+\Pi_{0} \bar{U}_{1} .
$$

We have imposed (4.4). For $k=1$, it yields $\partial_{t} \varphi_{1}+\bar{V}_{1}=0$ which allows to get $\varphi_{1}$. Observe that (4.3) with $k=1$ means that $W_{1}^{*} \equiv U_{1}^{*}$. Look at the oscillating part of (4.10) with the index $j=1$. Because of (4.3), the constraint on $W_{1}^{*}$ writes

$$
\partial_{t} W_{1}^{*}+\left(\boldsymbol{u}_{0} \cdot \nabla\right) W_{1}^{*}+\left(\partial_{t} \varphi_{\ell}+\bar{V}_{\ell}\right) \partial_{\theta} W_{1}^{*}=M W_{1}^{*}
$$

where $M$ is the linear application

$$
M U:=\left(\partial_{t} \Pi_{0}\right) U+\left(\left(\boldsymbol{u}_{0} \cdot \nabla\right) \Pi_{0}\right) U-\Pi_{0}(U \cdot \nabla) \boldsymbol{u}_{0} .
$$

Suppose that, for $j=1$, we have

$$
\partial_{t} \varphi_{j+k}+\bar{V}_{j+k}=0, \quad \forall k \in\{1, \ldots, \ell-1\} .
$$

TOME $134-2006-\mathrm{N}^{\mathrm{O}} 1$ 
In fact, in view of (4.4), this reduces to the last condition $\partial_{t} \varphi_{\ell}+\bar{V}_{\ell}=0$. Now, the link between $W_{1}^{*}$ and $\bar{V}_{\ell}$ is removed. It suffices to determine $W_{1}^{*}$ through the linear equation

$$
\partial_{t} W_{1}^{*}+\left(\boldsymbol{u}_{0} \cdot \nabla\right) W_{1}^{*}=M W_{1}^{*}, \quad W_{1}^{*}(0, x, \theta)=U_{10}^{*}(x, \theta) .
$$

Observe that the polarization condition $W_{1}^{*}=\Pi_{0} W_{1}^{*}$ is conserved since the equation on $W_{1}^{*}$ is equivalent to

$$
\left\{\begin{array}{l}
\Pi_{0}\left[\partial_{t} W_{1}^{*}+\left(\boldsymbol{u}_{0} \cdot \nabla\right) W_{1}^{*}+\left(W_{1}^{*} \cdot \nabla\right) \boldsymbol{u}_{0}\right]=0, \\
W_{1}^{*}=\Pi_{0} W_{1}^{*} .
\end{array}\right.
$$

Introduce the linear form

$$
\ell U:=\left|X_{0}\right|^{-2}\left[\partial_{t} X_{0} \cdot U+\left(\left(\boldsymbol{u}_{0} \cdot \nabla\right) X_{0}\right) \cdot U-X_{0} \cdot\left((U \cdot \nabla) \boldsymbol{u}_{0}\right)\right] .
$$

Consider the oscillating part of (4.9) with the index $j=1$. Since (4.3) imposes $V_{1}^{*} \equiv 0$, it remains

$$
P_{\ell+1}^{*}=\ell \partial_{\theta}^{-1} W_{1}^{*} \text {. }
$$

We must also have (4.12) with $j=1$, that is

$$
V_{\ell+1}^{*}=-\operatorname{div} \partial_{\theta}^{-1} W_{1}^{*} \text {. }
$$

At this stage, we know who is the profile $U_{1} \equiv \bar{U}_{1}+W_{1}^{*}$ and the pressure $P_{1} \equiv \bar{P}_{1}$. Moreover, by construction, we have the relations (4.13) and (4.14). Thus, hypothesis $\left(H_{1}\right)$ is verified.

The induction. - Suppose that the conditions given in $\left(H_{j}\right)$ are satisfied. The question is to obtain $\left(H_{j+1}\right)$. Consider first (4.11) with the index $j+1$. Use (4.14) and some integration by parts to get

$$
\left\langle V_{\ell+k}^{*} \partial_{\theta} U_{j+1-k}^{*}\right\rangle=\left\langle\operatorname{div} U_{k}^{*} \partial_{\theta} U_{j+1-k}^{*}\right\rangle, \quad \forall k \in\{1, \ldots, j\} .
$$

Thus, it remains

$$
\left\{\begin{aligned}
& \partial_{t} \bar{U}_{j+1}+\left(\boldsymbol{u}_{0} \cdot \nabla\right) \bar{U}_{j+1}+\left(\bar{U}_{j+1} \cdot \nabla\right) \boldsymbol{u}_{0}+\nabla \bar{P}_{j+1} \\
&+\sum_{k=1}^{j}\left(\bar{U}_{k} \cdot \nabla\right) \bar{U}_{j+1-k}+\sum_{k=1}^{j} \operatorname{div}\left\langle U_{k}^{*} \otimes U_{j+1-k}^{*}\right\rangle=0 \\
& \operatorname{div} \bar{U}_{j+1}=0 .
\end{aligned}\right.
$$

This system is completed with the initial data

$$
\bar{U}_{j+1}(0, x)=\bar{U}_{0(j+1)}(x), \quad \forall x \in \mathbb{R}^{d} .
$$

It gives access to $\bar{U}_{j+1}$ and $\bar{P}_{j+1}$. Recall that the step $j$ relies on (4.16). In particular, for $k=1$, we must have

$$
\partial_{t} \varphi_{j+1}+\left(\boldsymbol{u}_{0} \cdot \nabla\right) \varphi_{j+1}+X_{0} \cdot \bar{U}_{j+1}+\sum_{\ell=1}^{j} X_{\ell} \cdot \bar{U}_{j+1-\ell}=0
$$

BULletin DE LA SOCIÉtÉ MATHÉMATIQUE DE FRANCE 
Observe that, in the hypothesis $\left(H_{j+1}\right)$, this is exactly (4.13) with $k=j+1$. From this equation and

$$
\varphi_{j+1}(0, x)=\varphi_{0(j+1)}(x), \quad \forall x \in \mathbb{R}^{d},
$$

deduce $\varphi_{j+1}$. From $\varphi_{j+1}$, extract $X_{j+1}$ and $\Pi_{j+1}$. Impose the triangulation condition (4.16) written with the index $j+1$. It means that we add

$$
\partial_{t} \varphi_{j+\ell}+\bar{V}_{j+\ell}=0 \text {. }
$$

Then, extract the oscillating part of (4.10) written with $j+1$. Use $\left(H_{j}\right)$ and (4.20) in order to simplify the resulting equation. It yields

$$
\partial_{t} W_{j+1}^{*}+\left(\boldsymbol{u}_{0} \cdot \nabla\right) W_{j+1}^{*}=M W_{j+1}^{*}+f
$$

where $f$ is known. We get $W_{j+1}^{*}$ by solving (4.21). Therefore we have $U_{j+1}^{*}$ and we can deduce $V_{j+\ell+1}^{*}=-\operatorname{div} \partial_{\theta}^{-1} U_{j+1}^{*}$.

Now look at the constraint (4.9) for the index $j+1$. Extract the oscillating part. It allows to recover $P_{j+\ell+1}^{*}$. Thus we have $\left(H_{j+1}\right)$.

Apply the preceding induction up to $j=N-\ell$. In view of (i), (ii) and (iii) in Hypothesis $\left(H_{N-\ell}\right)$, the expressions

$$
U_{1}, \ldots, U_{N-\ell}, \quad P_{1}, \ldots, P_{N-\ell}, \quad \varphi_{1}, \ldots, \varphi_{N-\ell},
$$

are identified. In accordance with (4.13) and (4.14), define

$$
\begin{array}{lll}
V_{N-\ell+k}^{*}=-\operatorname{div} \partial_{\theta}^{-1} U_{N-2 \ell+k}^{*}, & W_{N-\ell+k}^{*} \equiv 0, & k \in\{1, \ldots, \ell\}, \\
\bar{V}_{N-\ell+k} \equiv 0, & \bar{W}_{N-\ell+k} \equiv 0, & k \in\{1, \ldots, \ell\}, \\
\varphi_{N-\ell+k}(t, x)=\varphi_{(N-\ell+k) 0}(x), & P_{N-\ell+k}=P_{N-\ell+k}^{*}, & k \in\{1, \ldots, \ell\} .
\end{array}
$$

An induction based on (4.2) allows to recover $U_{N-\ell+k}, \ldots, U_{N}$. Look at $u_{b}^{\varepsilon}$ and $p_{b}^{\varepsilon}$ as in (3.9). Note that we have (3.10) with

$$
f_{b}^{\varepsilon}(t, x, \theta)=\sum_{j=N+1-\ell}^{\infty} \varepsilon^{j / \ell} \mathcal{F}_{j}(t, x, \theta), \quad g_{b}^{\varepsilon}(t, x, \theta)=\sum_{j=N+1-\ell}^{\infty} \varepsilon^{j / \ell}\left(\operatorname{div} U_{j}+\partial_{\theta} V_{j+\ell}\right),
$$

where the sums are in fact finite and

$$
\begin{aligned}
\mathcal{F}_{j}(t, x, \theta):=-\sum_{k=0}^{j-1}\left(\partial_{t} \varphi_{\ell+k}+V_{\ell+k}\right) \partial_{\theta} U_{j-k}-\partial_{t} U_{j} \\
\quad-\sum_{k=0}^{j}\left(U_{k} \cdot \nabla\right) U_{j-k}-\nabla P_{j}-\sum_{k=0}^{j} \partial_{\theta} P_{\ell+k} \nabla \varphi_{j-k} .
\end{aligned}
$$

By construction, we have (i) and (ii) of Proposition 3.1, and also (3.11). The proof is therefore complete.

TOME $134-2006-\mathrm{N}^{\mathrm{O}} 1$ 
4.2. Proof of Theorem 3.1. - Now, we explain how to deduce Theorem 3.1 from Proposition 3.1. Select arbitrary initial data for

$$
\Pi_{0}(0, x) \widetilde{U}_{k}^{*}(0, x, \theta) \in H^{\infty}, \quad\left\langle\widetilde{U}_{k}\right\rangle(0, x) \in H^{\infty}, \quad 1 \leq k \leq N,
$$

and arbitrary initial data for

$$
\varphi_{k}(0, x) \in H^{\infty}, \quad 1 \leq k \leq \ell-1 .
$$

On the contrary, impose

$$
\varphi_{k}(0, .) \equiv 0, \quad \forall k \in\{\ell, \ldots, N\} .
$$

Proposition 3.1 provides with finite sequences

$$
\left\{\widetilde{U}_{k}\right\}_{1 \leq k \leq N}, \quad\left\{\widetilde{P}_{k}\right\}_{1 \leq k \leq N}, \quad\left\{\varphi_{k}\right\}_{1 \leq k \leq N},
$$

and source terms

$$
\tilde{f}_{b}^{\varepsilon}(t, x, \theta) \in \mathcal{W}_{T}^{\infty}, \quad \widetilde{g}_{b}^{\varepsilon}(t, x, \theta) \in \mathcal{W}_{T}^{\infty} .
$$

Define $\widetilde{\boldsymbol{u}}_{b}^{\varepsilon}$ and $\widetilde{\boldsymbol{p}}_{b}^{\varepsilon}$ as in (3.12). In the paragraph below, we eliminate the adjusting phase $\varphi_{a}^{\varepsilon}$ in order to get $\boldsymbol{u}_{b}^{\varepsilon}$ and $\boldsymbol{p}_{b}^{\varepsilon}$. We show that this operation creates only small remainders. We also check that this manipulation yields no contradiction when fixing the data at time $t=0$.

Dictionary between the profiles. - The functions $\widetilde{\boldsymbol{u}}_{b}^{\varepsilon}$ and $\widetilde{\boldsymbol{p}}_{b}^{\varepsilon}$ can also be written in terms of the phase $\varphi_{g}^{\varepsilon}$. Indeed, there is a unique decomposition

$$
\widetilde{\boldsymbol{u}}_{b}^{\varepsilon}=\check{\boldsymbol{u}}_{b}^{\varepsilon}+\boldsymbol{r} \boldsymbol{u}_{b}^{\varepsilon}=\check{\boldsymbol{u}}_{b}^{\varepsilon}+O\left(\varepsilon^{(N+1) / \ell}\right), \quad \widetilde{\boldsymbol{p}}_{b}^{\varepsilon}=\check{\boldsymbol{p}}_{b}^{\varepsilon}+\boldsymbol{r} \boldsymbol{p}_{b}^{\varepsilon}=\check{\boldsymbol{p}}_{b}^{\varepsilon}+O\left(\varepsilon^{(N+1) / \ell}\right),
$$

involving the representations

$$
\check{\boldsymbol{u}}_{b}^{\varepsilon}(t, x)=\check{u}_{b}^{\varepsilon}\left(t, x, \varepsilon^{-1} \varphi_{g}^{\varepsilon}(t, x)\right), \quad \check{\boldsymbol{p}}_{b}^{\varepsilon}(t, x)=\check{p}_{b}^{\varepsilon}\left(t, x, \varepsilon^{-1} \varphi_{g}^{\varepsilon}(t, x)\right)
$$

where the profiles $\check{u}_{b}^{\varepsilon}(t, x, \theta)$ and $\check{p}_{b}^{\varepsilon}(t, x, \theta)$ have the form

$$
\begin{aligned}
& \check{u}_{b}^{\varepsilon}(t, x, \theta)=\boldsymbol{u}_{0}(t, x)+\sum_{k=1}^{N} \varepsilon^{k / \ell} U_{k}(t, x, \theta), \\
& \check{p}_{b}^{\varepsilon}(t, x, \theta)=\boldsymbol{p}_{0}(t, x)+\sum_{k=1}^{N} \varepsilon^{k / \ell} P_{k}(t, x, \theta) .
\end{aligned}
$$

The transition from $\widetilde{\boldsymbol{u}}_{b}^{\varepsilon}$ to $\check{\boldsymbol{u}}_{b}^{\varepsilon}$ is achieved through the phase shift $\varphi_{a}^{\varepsilon}$

$$
\widetilde{U}_{k}\left(t, x, \varepsilon^{-1} \varphi_{b}^{\varepsilon}\right)=\widetilde{U}_{k}\left(t, x, \varepsilon^{-1} \varphi_{g}^{\varepsilon}+\varphi_{\ell}+\sum_{k=\ell+1}^{N} \varepsilon^{k / \ell-1} \varphi_{k}\right) .
$$

Use the Taylor formula in order to absorb the small term in the right. It furnishes the following explicit link between the $\left(U_{k}, P_{k}\right)$ and the $\left(\widetilde{U}_{k}, \widetilde{P}_{k}\right)$

$$
\left\{\begin{aligned}
U_{k}\left(t, x, \theta-\varphi_{\ell}(t, x)\right):=\widetilde{U}_{k}(t, x, \theta)+\mathcal{G}^{k}\left(\widetilde{U}_{1}, \ldots, \widetilde{U}_{k-1}\right)(t, x, \theta), \\
P_{k}\left(t, x, \theta-\varphi_{\ell}(t, x)\right):=\widetilde{P}_{k}(t, x, \theta)+\mathcal{G}^{k}\left(\widetilde{P}_{1}, \ldots, \widetilde{P}_{k-1}\right)(t, x, \theta) .
\end{aligned}\right.
$$

BULLETIN DE LA SOCIÉtÉ MATHÉMATIQUE DE FRANCE 
The application $\mathcal{G}^{k}$ can be put in the form

$$
\mathcal{G}^{k}\left(\widetilde{U}_{1}, \ldots, \widetilde{U}_{k-1}\right):=\sum_{p=1}^{k-1} \partial_{\theta}^{p} \mathcal{G}_{p}^{k}\left(\widetilde{U}_{1}, \ldots, \widetilde{U}_{k-p}\right), \quad k \in\{2, \ldots, N\} .
$$

The terms $\mathcal{G}_{p}^{k}$ are given by

$$
\mathcal{G}_{p}^{k}\left(\widetilde{U}_{1}, \ldots, \widetilde{U}_{k-p}\right):=\frac{1}{p !} \sum_{\alpha \in \mathcal{J}_{p}^{k}} \varphi_{\ell+1+\alpha_{1}} \times \cdots \times \varphi_{\ell+1+\alpha_{p}} \widetilde{U}_{\alpha_{p+1}},
$$

where the sum is taken over the set

$$
\begin{aligned}
\mathcal{J}_{p}^{k}:=\left\{\alpha=\left(\alpha_{1}, \ldots, \alpha_{p}, \alpha_{p+1}\right) \in \mathbb{N}^{p+1}\right. & \\
0 \leq \alpha_{j} \leq & N-\ell-1, \forall j \in\{1, \ldots, p\}, \\
& \left.1 \leq \alpha_{p+1} \leq k-p, \alpha_{1}+\cdots+\alpha_{p}+\alpha_{p+1}=k-p\right\} .
\end{aligned}
$$

The relation (4.24) and the definition of $\mathcal{G}^{k}$ imply that

$$
\bar{U}_{k}(t, x)=\left\langle\widetilde{U}_{k}\right\rangle(t, x), \quad \forall k \in\{1, \ldots, N\}, \forall t \in[0, T] .
$$

Therefore, prescribing the initial data for the $\bar{U}_{k}$ or the $\left\langle\widetilde{U}_{k}\right\rangle$ amounts to the same thing. The condition (4.22) yields

$$
\mathcal{G}_{p}^{k}\left(\widetilde{U}_{1}, \ldots, \widetilde{U}_{k-p}\right)(0, x, \theta)=0, \quad \forall k \in\{1, \ldots, N\} .
$$

Since $\varphi_{\ell}(0,.) \equiv 0$, we have

$$
\Pi_{0}(0, x) U_{k}^{*}(0, x, \theta)=\Pi_{0}(0, x) \widetilde{U}_{k}^{*}(0, x, \theta), \quad \forall k \in\{1, \ldots, N\} .
$$

In view of these identities, it is clearly equivalent to specify the initial data for the $\Pi_{0} U_{k}^{*}$ or the $\Pi_{0} \widetilde{U}_{k}^{*}$.

To get (3.7), we have also to replace $\widetilde{\boldsymbol{g}}_{b}^{\varepsilon}$ by zero. To this end, we first exhibit some properties of the operator 'div'.

The divergence free relation in the variables $(t, x)$ - Consider the application

$$
\operatorname{div}: C_{D}^{\infty}:=\{\mathbf{u} \subset D\} \longrightarrow \operatorname{Im}(\operatorname{div}) \subset\left\{\mathbf{g} \in H^{\infty} ; \widehat{\mathbf{g}}(0)=0\right\} .
$$

We can select some special right inverse.

Lemma 4.1. - There is a linear operator ridiv : $C_{D}^{\infty} \rightarrow H^{\infty}$ with

$$
\operatorname{div} \circ \operatorname{ridiv} \mathbf{g}=\mathbf{g}, \quad \forall \mathbf{g} \in \operatorname{Im}(\operatorname{div}) .
$$

For all $\iota>0$ and for all $m \in \mathbb{N}$, there is a constant $C_{m}^{\iota}>0$ such that

$$
\| \text { ridiv } \boldsymbol{g}\left\|_{H^{m}} \leq C_{m}^{\iota}\right\| \boldsymbol{g} \|_{H^{m+1+\frac{1}{2} d+\iota}}, \quad \forall \boldsymbol{g} \in \operatorname{Im}(\text { div }) .
$$

TOME $134-2006-\mathrm{N}^{\mathrm{O}} 1$ 
Proof of Lemma 4.1. - Introduce a cut-off function $\psi \in C^{\infty}\left(\mathbb{R}^{d}\right)$ such that

$$
\{\xi ; \psi(\xi) \neq 0\} \subset B(0,2], \quad\{\xi ; \psi(\xi)=1\} \supset B(0,1] .
$$

For $g \in \operatorname{Im}(\operatorname{div})$, take the explicit formula

$$
\operatorname{ridiv}(\boldsymbol{g}):=\mathcal{F}^{-1}\left(\int_{0}^{1} \nabla_{\xi}(\psi \widehat{\boldsymbol{g}})(r \xi) \mathrm{d} r+|\xi|^{-2}(1-\psi)(\xi) \widehat{\boldsymbol{g}}(\xi) \times \xi\right) .
$$

Since $\widehat{\boldsymbol{g}}(0)=0$, the relation (??) is satisfied. For $s>\frac{1}{2} d$, the injection $H^{s}\left(\mathbb{R}^{d}\right) \hookrightarrow L^{\infty}\left(\mathbb{R}^{d}\right)$ is continuous. It leads to $(4.27)$.

End of the proof of Theorem 3.1. - Take $\iota=1 / \ell>0$. Using (3.14) and Lemma 4.1, associated with the remark Finite speed of propagation in the next paragraph 5.1, we get

$$
\left\{\operatorname{ridiv} \widetilde{\boldsymbol{g}}_{b}^{\varepsilon}\right\}_{\varepsilon}=O\left(\varepsilon^{N / \ell-2-\frac{1}{2} d}\right)
$$

Define

Compute

$$
\begin{array}{ll}
\boldsymbol{c} \boldsymbol{u}_{b}^{\varepsilon}:=\boldsymbol{r} \boldsymbol{u}_{b}^{\varepsilon}-\operatorname{ridiv} \widetilde{\boldsymbol{g}}_{b}^{\varepsilon}, & \boldsymbol{c} \boldsymbol{p}_{b}^{\varepsilon}:=\boldsymbol{r} \boldsymbol{p}_{b}^{\varepsilon}, \\
\boldsymbol{u}_{b}^{\varepsilon}:=\check{\boldsymbol{u}}_{b}^{\varepsilon}+\boldsymbol{c} \boldsymbol{u}_{b}^{\varepsilon}, & \boldsymbol{p}_{b}^{\varepsilon}:=\check{\boldsymbol{p}}_{b}^{\varepsilon}+\boldsymbol{c} \boldsymbol{p}_{b}^{\varepsilon} .
\end{array}
$$

$$
\operatorname{div} \boldsymbol{u}_{b}^{\varepsilon}=\operatorname{div} \widetilde{\boldsymbol{u}}_{b}^{\varepsilon}-\operatorname{div} \circ \operatorname{ridiv} \widetilde{\boldsymbol{g}}_{b}^{\varepsilon}=0
$$

Moreover

$$
\partial_{t} \boldsymbol{u}_{b}^{\varepsilon}+\left(\boldsymbol{u}_{b}^{\varepsilon} \cdot \nabla\right) \boldsymbol{u}_{b}^{\varepsilon}+\nabla \boldsymbol{p}_{b}^{\varepsilon}=\boldsymbol{f}_{b}^{\varepsilon}
$$

with

$$
\begin{aligned}
\boldsymbol{f}_{b}^{\varepsilon}=\widetilde{\boldsymbol{f}}_{b}^{\varepsilon}-\left(\operatorname{ridiv} \widetilde{\boldsymbol{g}}_{b}^{\varepsilon} \cdot \nabla\right) \widetilde{\boldsymbol{u}}_{b}^{\varepsilon}-\left(\widetilde{\boldsymbol{u}}_{b}^{\varepsilon} \cdot \nabla\right) \operatorname{ridiv} \widetilde{\boldsymbol{g}}_{b}^{\varepsilon} \\
-\partial_{t} \operatorname{ridiv} \widetilde{\boldsymbol{g}}_{b}^{\varepsilon}+\left(\operatorname{ridiv} \widetilde{\boldsymbol{g}}_{b}^{\varepsilon} \cdot \nabla\right) \operatorname{ridiv} \widetilde{\boldsymbol{g}}_{b}^{\varepsilon} .
\end{aligned}
$$

A derivative in $t$ or $x$ costs a power of $\varepsilon$. The small error term $\boldsymbol{f}_{b}^{\varepsilon}$ is controled as indicated. The proof of Theorem 3.1 is therefore complete.

\section{Consequences of Theorem 3.1}

\subsection{Various comments}

The Leray projector. - Note $\Pi(\xi)$ the orthogonal projector from $\mathbb{R}^{d}$ onto the plane

$$
\xi^{\perp}:=\left\{u \in \mathbb{R}^{d} ; u \cdot \xi=0\right\} .
$$

Introduce the closed subspace

$$
\mathrm{F}:=\left\{\boldsymbol{u} \in L^{2} ; \operatorname{div} \boldsymbol{u}=0\right\} \subset L^{2} .
$$

Call $\mathfrak{P}$ the orthogonal projector from $L^{2}$ onto $\mathrm{F}$. It corresponds to the Fourier multiplier

$$
\mathfrak{P} \boldsymbol{u}=\Pi\left(D_{x}\right) \boldsymbol{u}:=(2 \pi)^{-\frac{1}{2} d} \int_{\mathbb{R}^{d}} \mathrm{e}^{i x \cdot \xi} \Pi(\xi) \widehat{\boldsymbol{u}}(\xi) \mathrm{d} \xi
$$

BULLETIN DE LA SOCIÉTÉ MATHÉMATIQUE DE FRANCE 
The application $\mathfrak{P}$ is the Leray projector onto the space of divergence free vector fields. It is a self-adjoint operator such that

$$
\operatorname{ker} \operatorname{div}=\operatorname{Im} \mathfrak{P}, \quad \operatorname{Im} \nabla=(\operatorname{ker}(\operatorname{div}))^{\perp}=\operatorname{ker} \mathfrak{P} .
$$

Consider the Cauchy problem

$$
\partial_{t} \boldsymbol{u}+\nabla \boldsymbol{p}=\boldsymbol{f}, \quad \operatorname{div} \boldsymbol{u}=0, \quad \boldsymbol{u}(0, .)=\boldsymbol{h}
$$

with data $\boldsymbol{f} \in L_{T}^{2}$ and $\boldsymbol{h} \in L^{2}$. It leads to the equivalent conditions

$$
\partial_{t} \boldsymbol{u}=\mathfrak{P} \boldsymbol{f}, \quad \boldsymbol{u}(0, .)=\mathfrak{P} \boldsymbol{h}, \quad \nabla \boldsymbol{p}=(\mathrm{Id}-\mathfrak{P}) \boldsymbol{f} .
$$

In particular, the equation (3.7) can be interpreted as

$$
\partial_{t} \boldsymbol{u}_{b}^{\varepsilon}+\mathfrak{P}\left(\left(\boldsymbol{u}_{b}^{\varepsilon} \cdot \nabla\right) \boldsymbol{u}_{b}^{\varepsilon}\right)=\mathfrak{P} \boldsymbol{f}_{b}^{\varepsilon}, \quad \boldsymbol{u}_{b}^{\varepsilon}(0, .)=\mathfrak{P} \boldsymbol{h}_{b}^{\varepsilon} .
$$

Infinite accuracy. - Fix any $\ell \in \mathbb{N}_{*}$. The Borel's summation process allows to take $N=+\infty$ in the Theorem 3.1. It yields BKW solutions $\left(\boldsymbol{u}_{b}^{\varepsilon}, \boldsymbol{p}_{b}^{\varepsilon}\right)$ which solve $(\mathcal{E})$ with infinite accuracy

$$
\partial_{t} \boldsymbol{u}_{b}^{\varepsilon}+\left(\boldsymbol{u}_{b}^{\varepsilon} \cdot \nabla\right) \boldsymbol{u}_{b}^{\varepsilon}+\nabla \boldsymbol{p}_{b}^{\varepsilon}=O\left(\varepsilon^{\infty}\right), \quad \operatorname{div} \boldsymbol{u}_{b}^{\varepsilon}=0 .
$$

Finite speed of propagation. - The characteristic curves of the field $\partial_{t}+\boldsymbol{u}_{0} \cdot \nabla_{x}$ are obtained by solving the differential equation

$$
\partial_{t} \Gamma(t, x)=\boldsymbol{u}_{0}(t, \Gamma(t, x)), \quad \Gamma(0, x)=x .
$$

Suppose that the oscillations of the profiles $U_{k 0}^{*}$ are concentrated in some domain $D \subset \mathbb{R}^{d}$. In other words

$$
\operatorname{supp}_{x} U_{k 0}^{*} \subset D, \quad \forall k \in\{1, \ldots, N\} .
$$

The BKW analysis (see (4.19)-(4.21)) reveals that for all $t \in[0, T]$ we have

$$
\operatorname{supp}_{x} U_{k}^{*}(t, .) \subset\{\Gamma(t, x) ; x \in D\}, \quad \forall k \in\{1, \ldots, N\} .
$$

The phenomena under study have a finite speed of propagation.

Large time existence. - Suppose that the function $\boldsymbol{u}_{0} \in \mathcal{W}_{\infty}^{\infty}$ is a global solution of the Euler equation. Suppose also that the phase $\varphi_{0} \in \mathcal{W}_{\infty}^{\infty}$ is subjected to $(3.5)$ on the strip $\left[0, \infty\left[\times \mathbb{R}^{d}\right.\right.$ and that it is a global solution of the eiconal equation. Fix any $T \in \mathbb{R}_{*}^{+}$. Since all the transport equations (4.19), (4.20) and (4.21) are linear, they can be solved on the whole time interval $[0, T]$. In particular, no blow up occurs at the level of the equations yielding the profiles $U_{k}$, $P_{k}$ and the phases $\varphi_{k}$. In this context, Theorem 3.1 can be applied with any $T \in \mathbb{R}_{*}^{+}$.

However, non linear effects are present. For instance, the appearance of the phases $\varphi_{k}$ with $k \geq 1$. We study some of the related mechanisms below.

5.2. The cascade of phases. - The phase $\varphi_{0}$ is determined as usual through the eiconal equation (3.3). We examine in this subsection 5.2 the part of the other phases coming into play.

TOME $134-2006-\mathrm{N}^{\mathrm{O}} 1$ 
The first phase shift. - First, suppose that $\varphi_{10} \equiv 0$ and $\bar{U}_{10} \equiv 0$. In view of (4.15), we have $\bar{U}_{1} \equiv 0$. Now, $\varphi_{1}$ is determined by

$$
\partial_{t} \varphi_{1}+\left(\boldsymbol{u}_{0} \cdot \nabla\right) \varphi_{1}+\bar{U}_{1} \cdot \nabla \varphi_{0}=\partial_{t} \varphi_{1}+\left(\boldsymbol{u}_{0} \cdot \nabla\right) \varphi_{1}=0 .
$$

It follows that $\varphi_{1} \equiv 0$. The terms $\varphi_{1}$ and $\bar{U}_{1}$ do not appear if we start with $\varphi_{10} \equiv 0$ and $\bar{U}_{10} \equiv 0$.

Suppose now that $\varphi_{10} \not \equiv 0$ or $\bar{U}_{10} \not \equiv 0$. Replace $\boldsymbol{u}_{00}$ by $\boldsymbol{u}_{00}+\delta \bar{U}_{10}$ and $\varphi_{00}$ by $\varphi_{00}+\delta \varphi_{10}$ where $\delta>0$ is some parameter. Solve (3.1)-(3.2) and (3.3)-(3.4) with these new groundstates. It furnishes expressions $\boldsymbol{u}_{0}$ and $\varphi_{0}$ which depend on $\delta$. Apply Theorem 3.1 where $\varphi_{10} \equiv 0$ and $\bar{U}_{10} \equiv 0$, while the other data are not changed. It yields $\varphi_{1} \equiv 0$ and $\bar{U}_{1} \equiv 0$. Then choose $\delta=\varepsilon$ to recover the situation under study.

This technical trick allows to reduce the case $\varphi_{10} \not \equiv 0$ or $\bar{U}_{10} \not \equiv 0$ to the case $\varphi_{10} \equiv 0$ and $\bar{U}_{10} \equiv 0$. It was already used in [8].

As explained below, such a manipulation is not possible concerning the other terms $\varphi_{2}, \ldots, \varphi_{\ell-1}$.

The second phase shift. - Suppose this time that

$$
\bar{U}_{10} \equiv 0, \quad \varphi_{10} \equiv 0, \quad \bar{U}_{20} \equiv 0, \quad \varphi_{20} \equiv 0 .
$$

Then, for $j=1$, the equation (4.19) becomes

$$
\left\{\begin{array}{l}
\partial_{t} \bar{U}_{2}+\left(\boldsymbol{u}_{0} \cdot \nabla\right) \bar{U}_{2}+\left(\bar{U}_{2} \cdot \nabla\right) \boldsymbol{u}_{0}+\nabla \bar{P}_{2}+\operatorname{div}\left\langle U_{1}^{*} \otimes U_{1}^{*}\right\rangle=0, \\
\operatorname{div} \bar{U}_{2}=0
\end{array}\right.
$$

This equation involves the source term $\operatorname{div}\left\langle U_{1}^{*} \otimes U_{1}^{*}\right\rangle$ which is able to awake the function $\bar{U}_{2}$. This influence can then be transmitted to $\varphi_{2}$ through the transport equation

$$
\partial_{t} \varphi_{2}+\left(\boldsymbol{u}_{0} \cdot \nabla\right) \varphi_{2}+\left(\bar{U}_{2} \cdot \nabla\right) \varphi_{0}=0 .
$$

As a matter of fact, we have

$$
\varphi_{2}(t, x)=-\frac{1}{2} \nabla \varphi_{00}(x) \cdot \partial_{t} \bar{U}_{2}(0, x) t^{2}+O\left(t^{3}\right)
$$

and there is no reason for the coefficient

$$
-\frac{1}{2} \nabla \varphi_{00}(x) \cdot \partial_{t} \bar{U}_{2}(0, x)=\frac{1}{2} \nabla \varphi_{00}(x) \cdot \mathfrak{P} \operatorname{div}\left\langle U_{10}^{*} \otimes U_{10}^{*}\right\rangle
$$

to be zero. To illustrate this assertion, consider the following simple case. Take $d=2$ and

$$
\boldsymbol{u}_{0} \equiv 0, \quad \varphi_{00}(x)=x_{1}, \quad U_{10}^{*}(x, \theta)=\psi(x)^{t}(0, h(\theta))
$$

where $\psi \in C^{\infty}\left(\mathbb{R}^{2} ; \mathbb{R}\right)$ and $h \in C^{\infty}(\mathbb{T} ; \mathbb{R})$ are two non trivial functions. Then, compute

$$
\nabla \varphi_{00}(x)=(1,0), \quad \operatorname{div}\left\langle U_{10}^{*} \otimes U_{10}^{*}\right\rangle={ }^{t}\left(0,\left\langle h(\theta)^{2}\right\rangle \partial_{2}\left[\psi(x)^{2}\right]\right) .
$$


For any scalar function $f$, the decomposition

$$
{ }^{t}(0, f)=\mathfrak{P}^{t}(0, f)+\nabla \chi
$$

implies that

$$
(1,0) \cdot \mathfrak{P}^{t}(0, f)=-\partial_{1} \chi, \quad \partial_{2} f=\Delta \chi .
$$

It follows that

$$
-\frac{1}{2} \nabla \varphi_{00}(x) \cdot \partial_{t} \bar{U}_{2}(0, x)=\frac{1}{2}\left\langle h(\theta)^{2}\right\rangle \partial_{1} \chi
$$

where the scalar function $\chi$ is subjected to

$$
\Delta \chi=-\partial_{22}^{2}\left[\psi(x)^{2}\right] .
$$

If $\psi$ has compact support, necessarily we have $\partial_{1} \chi \not \equiv 0$ which implies that $\varphi_{2} \not \equiv 0$. In general, the second phase shift $\varphi_{2}$ appears even if it is not present at time $t=0$. The phase $\varphi_{2}$ is generically created by the evolution.

The geometrical phase. - The other terms $\varphi_{3}, \ldots, \varphi_{\ell-1}$ are subjected to

$$
\partial_{t} \varphi_{k}+\boldsymbol{u}_{0} \cdot \nabla \varphi_{k}+\sum_{j=0}^{k-1}\left\langle\widetilde{U}_{k-j}\right\rangle \cdot \nabla \varphi_{j}=0, \quad \forall k \in\{3, \ldots, \ell-1\} .
$$

Like $\varphi_{2}$, the functions $\varphi_{3}, \ldots, \varphi_{\ell-1}$ are in general non trivial even if

$$
\varphi_{1}(0, .) \equiv \cdots \equiv \varphi_{\ell-1}(0, .) \equiv 0, \quad \bar{U}_{1}(0, .) \equiv \cdots \equiv \bar{U}_{\ell-1}(0, .) \equiv 0 .
$$

There is no more trick which allows to get rid of $\varphi_{2}, \cdots, \varphi_{\ell-1}$. The introduction of the phase shifts $\varphi_{k}$ with $2 \leq k \leq \ell-1$ cannot be avoided. Therefore the difficulties that we deal with appear from $\ell=3$. When $\ell \geq 3$, the characteristic rate $e$ of eddy dissipation is bigger than one [4]. This is the reason why such situations are refered to as turbulent regimes.

The expressions $\varphi_{2}, \ldots, \varphi_{\ell-1}$ are components of the geometrical phase

$$
\varphi_{g}^{\varepsilon}(t, x)=\varphi_{0}(t, x)+\sum_{k=1}^{\ell-1} \varepsilon^{k / \ell} \varphi_{k}(t, x)
$$

which comes from the approximate eiconal equation

$$
\partial_{t} \varphi_{g}^{\varepsilon}+\left(\left\langle\widetilde{u}_{b}^{\varepsilon}\right\rangle \cdot \nabla\right) \varphi_{g}^{\varepsilon}=O(\varepsilon) .
$$

The family $\left\{\boldsymbol{u}_{b}^{\varepsilon}(t, x)\right\}_{\varepsilon \in] 0,1]}$ has an $\varepsilon$-stratified regularity [20] with respect to the phase $\varphi_{g}^{\varepsilon}$. This is a geometrical information.

The complete phase $\varphi_{b}^{\varepsilon}$ yields a better approximation than $\varphi_{g}^{\varepsilon}$ since

$$
\partial_{t} \varphi_{b}^{\varepsilon}+\left(\left\langle\widetilde{u}_{b}^{\varepsilon}\right\rangle \cdot \nabla\right) \varphi_{b}^{\varepsilon}=O\left(\varepsilon^{(N+1) / \ell}\right) .
$$

The addition of the adjusting phase $\varphi_{a}^{\varepsilon}$ has no geometrical meaning. Nevertheless, it plays a crucial rôle as it is explained in the next paragraph.

TOME $134-2006-\mathrm{N}^{\mathrm{O}} 1$ 
5.3. Closure problems. - We have explained why appealing only to $\varphi_{0}$ is not sufficient. It turns out that BKW computations relying only on the geometrical phase $\varphi_{g}^{\varepsilon}$ come also to nothing. This is a subtle aspect when proving Theorem 3.1. We lay now stress on it. Define the transformations $\mathcal{G}^{k}$ as in (4.25)-(4.26). Fix $N \in \mathbb{N}_{*}$ and consider the map

$$
\begin{gathered}
\mathcal{G}:\left(\mathcal{W}_{T}^{\infty}\right)^{N} \longrightarrow\left(\mathcal{W}_{T}^{\infty}\right)^{N}, \\
\left(\begin{array}{c}
\widetilde{U}_{1} \\
\widetilde{U}_{2} \\
\vdots \\
\widetilde{U}_{N}
\end{array}\right)(t, x, \theta) \longmapsto\left(\begin{array}{c}
\widetilde{U}_{1} \\
\widetilde{U}_{2}+\mathcal{G}^{2}\left(\widetilde{U}_{1}\right) \\
\vdots \\
\widetilde{U}_{N}+\mathcal{G}^{N}\left(\widetilde{U}_{1}, \ldots, \widetilde{U}_{N-1}\right)
\end{array}\right)\left(t, x, \theta+\varphi_{\ell}(t, x)\right) .
\end{gathered}
$$

Obviously, the application $\mathcal{G}$ is one to one. There is a complete dictionary between the $U_{j}$ and the $\widetilde{U}_{j}$. Once the $U_{j}$ or the $\widetilde{U}_{j}$ are known, it is entirely equivalent to use the representation $\boldsymbol{u}_{b}^{\varepsilon}$ or $\widetilde{\boldsymbol{u}}_{b}^{\varepsilon}$. Before the $U_{j}$ or the $\widetilde{U}_{j}$ have been identified, that is in practice, for instance when performing the BKW calculus, it is deeply different to employ $\boldsymbol{u}_{b}^{\varepsilon}$ or $\widetilde{\boldsymbol{u}}_{b}^{\varepsilon}$. Indeed, there is a unique choice of the $\varphi_{k}$ with $\ell \leq k \leq N$, which imposes a specific hierarchy between the profiles $\widetilde{U}_{k}$, which makes possible the triangulation of the equations obtained by the formal computations.

Let us explain this affirmation more precisely. In the subsection 4.1, we have performed the BKW analysis with the profiles $\widetilde{U}_{k}$. The result was a sequence of equations

$$
\widetilde{X}^{k}\left(\widetilde{U}_{1}, \ldots, \widetilde{U}_{k+\ell}\right)=0, \quad 1 \leq k \leq N .
$$

As usual in non linear geometric optics, this can be rewritten in order to find a sequence of well-posed equations

$$
\dot{X}^{k}\left(\dot{U}_{k}\right)=\mathcal{F}\left(\dot{U}_{1}, \ldots, \dot{U}_{k-1}\right), \quad 1 \leq k \leq N,
$$

where the $\dot{U}_{k}$ are made of pieces of the $\widetilde{U}_{j}$ (in the circumstances $\bar{U}_{j}, \bar{P}_{j}, \varphi_{j}$, $W_{j}^{*}, V_{j+\ell}^{*}$ and $\left.P_{j+\ell}^{*}\right)$. Of course, the equation (5.4) can be interpreted in terms of the $\widetilde{U}_{j}$ and then (using $\mathcal{G}$ ) in terms of the $U_{j}$. However, in this second step, something unusual happens. The access to $U_{j}$ requires to implement $\widetilde{U}_{j}$, the phase shift $\varphi_{\ell}$ and the transformations $\mathcal{G}_{p}^{j}$ with $1 \leq p \leq j-1$. Now, to compute $\mathcal{G}_{1}^{j}$, we need to identify $\varphi_{j+\ell-1}$ which is not included in $\dot{U}_{j}$. This is what says (4.24)-(4.25).

In other words, our analysis reveals that $\varphi_{\ell}$ or the various coefficients $\varphi_{i}$ which appear in $(4.26)$ do not depend only on $\left(\dot{U}_{1}, \ldots, \dot{U}_{k}\right)$ but also on some $\dot{U}_{i}$ with $i>k$. Therefore, the interpretation of (5.4) in terms of the $U_{j}$ yields some underdetermined system. Computations involving the functions $U_{j}$ lead to a sequence of equations which are not closed. 
The insertion of the phases $\varphi_{k}$ with $\ell \leq k \leq N$ is an elegant way to introduce $\mathcal{G}$. The change of variables $\mathcal{G}$, though it is a function of $\left(U_{1}, \ldots, U_{N}\right)$, is needed to progress. It allows to get round closure problems.

5.4. Obvious instabilities. - The obvious instabilities are the mechanisms of amplifications which can be detected by looking directly at the formal expansions $\boldsymbol{u}_{b}^{\varepsilon}$. They imply the non linear instability of Euler equations. Indeed, fix any $T>0$, any $\boldsymbol{u}_{0} \in \mathcal{W}_{T}^{\infty}\left(\mathbb{R}^{d}\right)$ which is solution of $(\mathcal{E})$, and any $\delta>0$. Work on the balls

$$
\begin{aligned}
& B_{0}\left(\boldsymbol{u}_{0} ; \delta\right]:=\left\{\boldsymbol{u} \in L^{2} ;\left\|\boldsymbol{u}(.)-\boldsymbol{u}_{0}(0, .)\right\|_{L^{2}\left(\mathbb{R}^{d}\right)} \leq \delta\right\}, \\
& B_{T}\left(\boldsymbol{u}_{0} ; \delta\right]:=\left\{\boldsymbol{u} \in L_{T}^{2} ;\left\|\boldsymbol{u}-\boldsymbol{u}_{0}\right\|_{L^{2}\left([0, T] \times \mathbb{R}^{d}\right)} \leq \delta\right\} .
\end{aligned}
$$

Proposition 5.1. - For all constant $C>0$, there are small data

$$
(\boldsymbol{h}, \widetilde{\boldsymbol{h}}) \in\left(B_{0}\left(\boldsymbol{u}_{0} ; \delta\right] \cap H^{\infty}\right)^{2}, \quad(\boldsymbol{f}, \widetilde{\boldsymbol{f}}) \in\left(B_{T}\left(\boldsymbol{u}_{0} ; \delta\right] \cap \mathcal{W}_{T}^{\infty}\right)^{2}
$$

so that the Cauchy problems

$$
\begin{array}{rlrl}
\partial_{t} \boldsymbol{u}+(\boldsymbol{u} \cdot \nabla) \boldsymbol{u}+\nabla \boldsymbol{p} & =\boldsymbol{f}, & \operatorname{div} \boldsymbol{u}=0, & \boldsymbol{u}(0, .)=\boldsymbol{h}(.), \\
\partial_{t} \widetilde{\boldsymbol{u}}+(\widetilde{\boldsymbol{u}} \cdot \nabla) \widetilde{\boldsymbol{u}}+\nabla \widetilde{\boldsymbol{p}}=\widetilde{\boldsymbol{f}}, & \operatorname{div} \widetilde{\boldsymbol{u}}=0, & \widetilde{\boldsymbol{u}}(0, .)=\widetilde{\boldsymbol{h}}(.),
\end{array}
$$

have solutions $(\boldsymbol{u}, \widetilde{\boldsymbol{u}}) \in B_{T}\left(\boldsymbol{u}_{0} ; \delta\right]^{2}$ and there is $\left.\left.t \in\right] 0, T\right]$ such that

$$
\begin{aligned}
& \|(\boldsymbol{u}-\widetilde{\boldsymbol{u}})(t, .)\|_{L^{2}\left(\mathbb{R}^{d}\right)} \\
& \quad \geq C\left(\|\boldsymbol{h}-\widetilde{\boldsymbol{h}}\|_{L^{2}\left(\mathbb{R}^{d}\right)}+\int_{0}^{t}\|(\boldsymbol{f}-\tilde{\boldsymbol{f}})(s, .)\|_{L^{2}\left(\mathbb{R}^{d}\right)} \mathrm{d} s\right) .
\end{aligned}
$$

Inequalities as (5.5) are well-known. In general, see [8], [17], [19], the proof is achieved in two steps:

- First detect equilibria where instability arises in the discrete spectrum.

- Then establish that linearized instability implies non linear instability.

The procedure we adopt below is different. We just look at approximate solutions like $\boldsymbol{u}_{b}^{\varepsilon}$. It follows a more simple proof of inequality (5.5). In fact, the Proposition 5.1 is a convenient way to retrieve known non linear instability results.

Proof of Proposition 5.1. - The proof consists in a contradiction argument based on the decomposition of Theorem 3.1. Take $\ell=2$ and $N \geq(8+d)$. Consider two deals of initial data

$$
\begin{array}{ccc}
\widetilde{U}_{k}^{1}(0, x, \theta), & \varphi_{k}^{1}(0, x), & 1 \leq k \leq N, \\
\widetilde{U}_{k}^{2}(0, x, \theta), & \varphi_{k}^{2}(0, x), & 1 \leq k \leq N .
\end{array}
$$

Fix these expressions in the following way

$$
\widetilde{U}_{1}^{1}(0, .) \equiv \widetilde{U}_{1}^{2}(0, .), \quad \varphi_{1}^{1}(0, .) \equiv \varphi_{1}^{2}(0, .) \equiv 0, \quad \varphi_{2}^{1}(0, .) \equiv \varphi_{2}^{2}(0, .) \equiv 0 .
$$

TOME $134-2006-\mathrm{N}^{\mathrm{O}} 1$ 
It implies that

$$
\widetilde{U}_{1}^{1}(t, .) \equiv \widetilde{U}_{1}^{2}(t, .), \quad \varphi_{1}^{1}(t, .) \equiv \varphi_{1}^{2}(t, .) \equiv 0, \quad \forall t \in[0, T] .
$$

Adjust $\widetilde{U}_{2}^{1}(0,$.$) and \widetilde{U}_{2}^{2}(0,$.$) so that$

$$
\partial_{t}\left(\varphi_{2}^{1}-\varphi_{2}^{2}\right)(0, .)=-\nabla \varphi_{0} \cdot\left\langle\widetilde{U}_{2}^{1}-\widetilde{U}_{2}^{2}\right\rangle(0, .) \not \equiv 0 .
$$

Therefore, we are sure to find some $t>0$ such that $\left(\varphi_{2}^{1}-\varphi_{2}^{2}\right)(t,.) \not \equiv 0$. It follows that

$$
\begin{aligned}
U_{1}^{1}(t, x, \theta)= & \widetilde{U}_{1}^{1}\left(t, x, \theta+\varphi_{2}^{1}(t, x)\right) \\
& \not \equiv U_{1}^{2}(t, x, \theta)=\widetilde{U}_{1}^{1}\left(t, x, \theta+\varphi_{2}^{2}(t, x)\right) .
\end{aligned}
$$

Note $\boldsymbol{u}_{b}^{\varepsilon 1}$ and $\boldsymbol{u}_{b}^{\varepsilon 2}$ the approximate solutions built with the profiles $\left\{U_{k}^{1}\right\}_{k}$ and $\left\{U_{k}^{2}\right\}_{k}$. The associated error terms are $\boldsymbol{f}_{b}^{\varepsilon 1}$ and $\boldsymbol{f}_{b}^{\varepsilon 2}$. Now, proceed by contradiction. Suppose that Proposition 5.1 is wrong. Then, there is $C>0$ and $\left.\left.\varepsilon_{1} \in\right] 0, \varepsilon_{0}\right]$ such that for all $\left.\left.\varepsilon \in\right] 0, \varepsilon_{1}\right]$, we have

$$
\begin{aligned}
\left\|\left(\boldsymbol{u}_{b}^{\varepsilon 1}-\boldsymbol{u}_{b}^{\varepsilon 2}\right)(t, .)\right\|_{L^{2}\left(\mathbb{R}^{d}\right)} \leq C\left(\left\|\left(\boldsymbol{u}_{b}^{\varepsilon 1}-\boldsymbol{u}_{b}^{\varepsilon 2}\right)(0, .)\right\|_{L^{2}\left(\mathbb{R}^{d}\right)}\right. & \\
& \left.+\int_{0}^{t}\left\|\left(\boldsymbol{f}_{b}^{\varepsilon 1}-\boldsymbol{f}_{b}^{\varepsilon 2}\right)(s, .)\right\|_{L^{2}\left(\mathbb{R}^{d}\right)} \mathrm{d} s\right) .
\end{aligned}
$$

Divide this inequality by $\sqrt{\varepsilon}$. By construction, we have

$$
\begin{aligned}
& \varepsilon^{-\frac{1}{2}}\left\|\left(\boldsymbol{u}_{b}^{\varepsilon 1}-\boldsymbol{u}_{b}^{\varepsilon 2}\right)(0, .)\right\|_{L^{2}\left(\mathbb{R}^{d}\right)}=O(\sqrt{\varepsilon}), \\
& \varepsilon^{-\frac{1}{2}}\left\|\left(\boldsymbol{f}_{b}^{\varepsilon 1}-\boldsymbol{f}_{b}^{\varepsilon 2}\right)(s, .)\right\|_{L^{2}\left(\mathbb{R}^{d}\right)}=O(\sqrt{\varepsilon}), \quad \forall s \in[0, t] \text {. } \\
& \varepsilon^{-\frac{1}{2}}\left\|\left(\boldsymbol{u}_{b}^{\varepsilon 1}-\boldsymbol{u}_{b}^{\varepsilon 2}\right)(t, .)\right\|_{L^{2}\left(\mathbb{R}^{d}\right)}=\|\left(U_{1}^{1}-U_{1}^{2}\right)\left(t, \cdot, \varepsilon^{-1} \varphi_{g}^{\varepsilon}(t, .) \|_{L^{2}\left(\mathbb{R}^{d}\right)}+O(\sqrt{\varepsilon})\right. \text {. }
\end{aligned}
$$

It follows that

$$
\lim _{\varepsilon \rightarrow 0} \varepsilon^{-\frac{1}{2}}\left\|\left(\boldsymbol{u}_{b}^{\varepsilon 1}-\boldsymbol{u}_{b}^{\varepsilon 2}\right)(t, .)\right\|_{L^{2}\left(\mathbb{R}^{d}\right)}=\left\|\left(U_{1}^{1}-U_{1}^{2}\right)(t, .)\right\|_{L^{2}\left(\mathbb{R}^{d} \times \mathbb{T}\right)}=0
$$

which is inconsistent with (5.6).

In the proof presented above, the amplification is due to $\varphi_{2}$ which is the principal term in the adjusting phase. The presence of $\varphi_{2}$ becomes efficient in comparison with the other effects when

$$
\left|\widetilde{U}_{1}^{1}\left(t, x, \theta+\varphi_{2}^{1}(t, x)\right)-\widetilde{U}_{1}^{2}\left(t, x, \theta+\varphi_{2}^{2}(t, x)\right)\right| \sim c t \gg \sqrt{\varepsilon} .
$$

This requires to wait a lapse of time bigger than $\sqrt{\varepsilon}$. This delay can be reduced by adapting the above procedure to the cases $\ell>2$.

5.5. The mathematical background. - This subsection is mainly heuristical. It describes consequences of Theorem 3.1. Since it contains assertions which are involved in the paragraph 4.1 , it could be difficult to read if one does not have in mind details of the proof of Proposition 3.1.

BULletin DE LA SOCIÉtÉ MATHÉMATIQUE DE FRANCE 
Microstructures. - The result 3.1 is concerned with the convection of microstructures. It is linked with the multiple scale approach of [26] and [4]. In [26] the authors look for BKW solutions $\boldsymbol{u}_{\dagger}^{\varepsilon}$ in the form

$$
\boldsymbol{u}_{\dagger}^{\varepsilon}(t, x)=\boldsymbol{u}_{0}(t, x)+U_{0}^{*}\left(t, x, \varepsilon^{-1} t, \varepsilon^{-1} \vec{\varphi}_{0}(t, x)\right)+O(\varepsilon) .
$$

In the more recent paper [4], the selected expansion is

$$
\mu_{\dagger}^{\varepsilon}(t, x)=\boldsymbol{u}_{0}(t, x)+\varepsilon^{\frac{1}{3}} U_{1}\left(t, x, \varepsilon^{-\frac{2}{3}} t, \varepsilon^{-1} \vec{\varphi}_{0}(t, x)\right)+O\left(\varepsilon^{\frac{2}{3}}\right) .
$$

Both articles [4] and [26] use homogenization techniques. They perform computations involving expressions as $\boldsymbol{u}_{\dagger}^{\varepsilon}$ or $\mu_{\dagger}^{\varepsilon}$. Simplifications (supported by engineering experiments) are made in order to get effective equations for the evolution of $\left(\boldsymbol{u}_{0}, U_{0}^{*}\right)$ or $\left(\boldsymbol{u}_{0}, U_{1}\right)$.

Consider the simple case of one phase expansions (that is when $\vec{\varphi}_{0} \equiv \varphi_{0}$ is a scalar valued function). Reasons why a complete mathematical analysis based on $\boldsymbol{u}_{\dagger}^{\varepsilon}$ or $\mu_{\dagger}^{\varepsilon}$ is not available can be drawn from Theorem 3.1. For instance, look at $\mu_{\dagger}^{\varepsilon}$. When $\ell=3$, the oscillation $\mu_{\dagger}^{\varepsilon}$ involves the same scales as $\boldsymbol{u}_{(3, N)}^{\varepsilon}$ since

$$
\varepsilon^{-1} \varphi_{g}^{\varepsilon}(t, x)=\varepsilon^{-1} \varphi_{0}(t, x)+\varepsilon^{-\frac{2}{3}} \varphi_{1}(t, x)+\varepsilon^{-\frac{1}{3}} \varphi_{2}(t, x) .
$$

Now the analogy stops here since in general $\varphi_{1}(t, x) \not \equiv t$ and $\varphi_{2}(t, x) \not \equiv 0$. These are geometrical obstructions which prevent to describe the propagation by way of $\mu_{\dagger}^{\varepsilon}$. The asymptotic expansion $\mu_{\dagger}^{\varepsilon}$ is not suitable.

Analogous arguments concerning $\boldsymbol{u}_{\dagger}^{\varepsilon}$ will be presented further.

Compensated compactness. — Consider approximate solutions $\left(\boldsymbol{u}_{b}^{\varepsilon}, \boldsymbol{p}_{b}^{\varepsilon}\right)$ with infinite accuracy. They satisfy

$$
\partial_{t} \boldsymbol{u}_{b}^{\varepsilon}+\left(\boldsymbol{u}_{b}^{\varepsilon} \cdot \nabla\right) \boldsymbol{u}_{b}^{\varepsilon}+\nabla \boldsymbol{p}_{b}^{\varepsilon}=\boldsymbol{f}_{b}^{\varepsilon}=O\left(\varepsilon^{\infty}\right), \quad \operatorname{div} \boldsymbol{u}_{b}^{\varepsilon}=0 .
$$

Suppose that $\boldsymbol{u}_{00} \equiv 0$ so that

$$
\boldsymbol{u}_{b}^{\varepsilon}(0, .)=\boldsymbol{h}_{b}^{\varepsilon}(.)=O\left(\varepsilon^{1 / \ell}\right) .
$$

Now, forget all about the explicit construction of Theorem 3.1. Since the $L^{2}-$ norm is conserved, any smooth solution of (5.7) is subjected to the uniform control

$$
\left.\left.\sup \left\{\left\|\varepsilon^{-1 / \ell} \boldsymbol{u}_{b}^{\varepsilon}\right\|_{L_{T}^{2}} ; \varepsilon \in\right] 0,1\right]\right\} \leq C<\infty .
$$

Arguments issued from the theory of compensated compactness [18] can be employed to study the family $\left\{\varepsilon^{-1 / \ell} \boldsymbol{u}_{b}^{\varepsilon}\right\}_{\varepsilon}$. In the spirit of [12] or [14], we can try to exploit the informations contained in (5.9) and the equation on $\boldsymbol{u}_{b}^{\varepsilon}$ in order to describe the asymptotic behaviour when $\varepsilon$ goes to zero of the functions $\varepsilon^{-1 / \ell} \boldsymbol{u}_{b}^{\varepsilon}$.

However this approach is not applicable here.

Indeed, obstructions come from the presence of obvious instabilities. Below, we recall the intuitive idea of what can happen. Use the representation $\widetilde{\boldsymbol{u}}_{b}^{\varepsilon}$

TOME $134-2006-\mathrm{N}^{\mathrm{O}} 1$ 
involving the phase $\varphi_{b}^{\varepsilon}$. The determination of the intermediate term $\varphi_{\ell}$ requires to identify $\left\langle\widetilde{U}_{\ell}\right\rangle$ and $\widetilde{U}_{\ell-1}^{*}$. This is a consequence of the equations (4.13) and (4.19).

In view of the formula (4.24), when $\varphi_{\ell}$ is modified by an amount of $\delta \varphi_{\ell}$, the quantity $U_{1}(t, x, \theta)$ undergoes a perturbation of the same order $\delta \varphi_{\ell}$. When dealing with quasi-singularities, some quantities with $\varepsilon$ in factor (like $\left\langle\widetilde{U}_{\ell}\right\rangle$ ) or with $\varepsilon^{1-1 / \ell}$ in factor (like $\widetilde{U}_{\ell-1}^{*}$ ) can control informations of size $\varepsilon^{1 / \ell}$. This fact is expressed by the following rules of transformation

$$
\left\{\begin{array}{l}
\left\langle\widetilde{U}_{\ell}\right\rangle /\left\langle\widetilde{U}_{\ell}\right\rangle+\delta\left\langle\widetilde{U}_{\ell}\right\rangle \\
\widetilde{U}_{\ell-1}^{*} / \widetilde{U}_{\ell-1}^{*}+\delta \widetilde{U}_{\ell-1}^{*} \Longrightarrow \boldsymbol{u}_{b}^{\varepsilon} / \boldsymbol{u}_{b}^{\varepsilon}+O\left(\varepsilon^{1 / \ell}\right) \delta\left\langle\widetilde{U}_{\ell}\right\rangle, \\
\boldsymbol{u}_{b}^{\varepsilon}+O\left(\varepsilon^{1 / \ell}\right) \delta \widetilde{U}_{\ell}^{*} .
\end{array}\right.
$$

Now reverse the preceding reasoning. To describe features in the principal oscillating term $\varepsilon^{1 / \ell} U_{1}^{*}\left(t, x, \varepsilon^{-1} \varphi_{g}^{\varepsilon}(t, x)\right)$, we must identify $\varphi_{\ell}$ which means to obtain $\left\langle\widetilde{U}_{\ell}\right\rangle$ and $\widetilde{U}_{\ell-1}^{*}$. In other words, we need to know quantities which have respectively $\varepsilon$ and $\varepsilon^{1-1 / \ell}$ in factor. When $\ell \geq 2$ such informations are clearly not reachable by rough controls as (5.9).

The preceding discussion indicates that the study of turbulent regimes requires to combine at least geometrical aspects, multiphase analysis and high order expansions. The tools of non linear geometric optics seem to be appropriate. Some attempts in this direction have already been made.

Non linear geometric optics. - We make in this paragraph several comments about non linear geometric optics. They concern both old [21], [20], [28] and more recent [7], [8], [9] results which all are devoted to one phase expansions of the type

$$
\boldsymbol{u}_{\natural}^{\varepsilon}(t, x):=\boldsymbol{u}_{0}(t, x)+\sum_{k=k_{0}}^{\infty} \varepsilon^{k / \ell} U_{k}\left(t, x, \varepsilon^{-1} \varphi_{0}(t, x)\right)
$$

where $k_{0} \in \mathbb{N}$ and $\ell \in \mathbb{N}_{*}$.

$\triangleright$ Systems of multidimensional conservation laws Consider the hyperbolic non linear system

$$
\partial_{t} f_{0}(u)+\sum_{j=1}^{d} \partial_{j} f_{j}(u)=0, \quad u \in \mathbb{R}^{m}, m \in \mathbb{N}_{*}
$$

where the fluxes $f_{j}: \mathbb{R}^{m} \rightarrow \mathbb{R}^{m}$ are smooth functions. Introduce the matrix symbol

$$
A(u, \xi):=\sum_{j=1}^{d} \xi_{j} f_{0}^{\prime}(u)^{-1} f_{j}^{\prime}(u), \quad(u, \xi) \in \mathbb{R}^{m} \times \mathbb{R}^{d} .
$$

Select some eigenvalue $\lambda(u, \xi)$ of $A(u, \xi)$. We have

$$
\mathbb{E}(u, \xi):=\operatorname{ker}[A(u, \xi)-\lambda(u, \xi) \operatorname{Id}] \neq\{0\} .
$$

BULletin DE LA SOCIÉtÉ MATHÉMATIQUE DE FRANCE 
Introduce the vector space

A change of coordinates $[9]$ yields

$$
\mathbb{F}(u):=\bigcap_{\xi \in \mathbb{R}^{d} \backslash\{0\}} \mathbb{E}(u, \xi) .
$$

$$
\mathbb{F}(u)=\mathbb{F}, \quad \forall u \in \mathbb{R}^{m} .
$$

Suppose that some of the oscillation $\boldsymbol{u}_{\natural}^{\varepsilon}$ is polarized in the direction of $\mathbb{E}$ which means that the projection of $\partial_{\theta} U_{k 0}^{*}(t, x, \theta)$ on $\mathbb{E}\left(\boldsymbol{u}_{0}(t, x)\right)$ is not always reduced to $\{0\}$. The problem is to determine $\left(k_{0}, \ell\right)$ with $k_{0} / \ell$ as minimal as possible such that approximate (or better exact) solutions like (5.11) exist on some uniform interval $[0, T]$ with $T>0$.

$\alpha$ ) When $\lambda$ is genuine non linear, because of the formation of shocks, the pertinent regime is $k_{0}=1$ and $\ell=1$. This is the domain of weakly non linear geometric optics. The asymptotic behavior and the stability of $\boldsymbol{u}_{\natural}^{\varepsilon}$ are well understood. In fact, a complete theory has been achieved (see [21], [20] and the related references).

$\beta$ ) When $\lambda$ is linearly degenerate, we can take $k_{0}=1$ and $\ell=2$. Expressions as $\boldsymbol{u}_{\natural}^{\varepsilon}$ are called strong oscillations. In the hyperbolic situation, the family $\left\{\boldsymbol{u}_{\natural}^{\varepsilon}\right\}_{\varepsilon \in] 0,1]}$ is unstable [8] on the interval [0,T]. It becomes stable on condition that a small viscosity is incorporated [7]. Applications can be given to describe large-scale motions in the atmosphere [7].

$\gamma$ ) When $\lambda$ is linearly degenerate, when $\mathbb{F} \neq\{0\}$, and when the oscillations are supported by $\mathbb{F}$, the choice $k_{0}=0$ and $\ell=1$ is suitable. We can construct large amplitude high frequency waves [9].

Nonisentropic compressible Euler equations are the prototype of a non linear hyperbolic system at the level of which the three situations $\alpha$ ), $\beta$ ) and $\gamma$ ) can be tested. Singularities which correspond to the generation of shocks by compression [29] can appear (as in $\alpha$ ). There is a linearly degenerate eigenvalue (as in $\beta$ ). The vector space $\mathbb{F}$ is non trivial (as in $\gamma$ ): it is one-dimensional and corresponds to the entropy component.

The above study is not exhaustive. For instance, it does not include:

$\delta)$ Multidimensional Bürgers type equations

$$
\partial_{t} u+\sum_{j=1}^{d} a_{j}(u) \partial_{j} u=0, \quad u \in \mathbb{R}^{m},
$$

where the scalar coefficients $a_{j}: \mathbb{R}^{m} \rightarrow \mathbb{R}$ are all non constant functions. The quasilinear system (5.12) is not always issued from an equation in conservative form. Moreover, though $\mathbb{F}=\mathbb{R}^{d}$, the eigenvalue

$$
\lambda(u, \xi)=\sum_{j=1}^{d} \xi_{j} a_{j}(u)
$$

TOME $134-2006-\mathrm{N}^{\mathrm{O}} 1$ 
is not linearly degenerate since

$$
\forall(u, \xi) \in \mathbb{R}^{m} \times\left(\mathbb{R}^{d} \backslash\{0\}\right), \exists \widetilde{u} \in E(u, \xi) \equiv \mathbb{R}^{d}, \quad \nabla \lambda(u, \xi) \cdot \widetilde{u} \neq 0 .
$$

There is no systematic study in such cases. Special compatibility conditions seem to be needed in order to progress up to $k_{0}=0$ and $\ell=1$.

Likewise, the above classification does not include:

$\triangleright$ Incompressible Euler equations. - Here, there is no genuine shock and the production of singularities poses a much more subtle problem [2], [10] which up to now remains basically open.

Theorem 3.1 says that, for $k_{0}=1$, one can reach any $\ell \in \mathbb{N}_{*}$. In this sense, the situation is intermediate between $\beta$ ) and $\gamma$ ). Aspects of $\delta$ ) come also into play. But these analogies must be handled with care. In fact, the incompressible framework is quite apart.

$\triangleright$ Brief survey. - In the preceding approach, the choice for the amplitude of the oscillations was very important. Another way to present the analysis is to fix the size of the oscillating initial data. For example, we can start with large amplitude high frequency waves

$$
\boldsymbol{u}_{\infty}^{\varepsilon}(0, x)=U_{0}\left(0, x, \varepsilon^{-1} \varphi_{0}(0, x)\right)+O(\varepsilon), \quad \partial_{\theta} U_{0}^{*}(0, .) \not \equiv 0 .
$$

The idea is to increase the time of propagation $T_{\varepsilon}$ to reach the regime where non linear effects appear. The above discussion can then be summarized by the diagram of Figure1. This picture allows to understand the position of the

\begin{tabular}{|c|c|c|c|}
\hline & $\begin{array}{c}\text { infinite cascade } \\
\text { of phases } \\
\varphi_{0}-\left(\varphi_{1}\right)-\cdots\end{array}$ & $\begin{array}{l}\text { turbulert } \\
\text { flows }\end{array}$ & $\begin{array}{l}\text { incompressible } \\
\text { fluid equations }\end{array}$ \\
\hline$T \simeq \varepsilon^{\frac{1}{3}}$ & $\varphi_{0}-\left(\varphi_{1}\right)-\varphi_{2}$ & turbulent flows & $\begin{array}{l}\text { incompressible } \\
\text { fluid equations }\end{array}$ \\
\hline$T \simeq \varepsilon^{\frac{1}{2}}$ & $\varphi_{0}-\left(\varphi_{1}\right)$ & $\begin{array}{c}\text { strong } \\
\text { oscillations } \\
{[\mathbf{7}],[\mathbf{9}]}\end{array}$ & $\begin{array}{c}\text { systems of conservation } \\
\text { laws with a linearly } \\
\text { degenerate field }\end{array}$ \\
\hline$T \simeq \varepsilon$ & $\varphi_{0}$ & $\begin{array}{c}\text { weakly non linear } \\
\text { geometric optics } \\
{[\mathbf{2 1}],[\mathbf{2 0}]}\end{array}$ & $\begin{array}{c}\text { systems of } \\
\text { conservation laws }\end{array}$ \\
\hline$T=0$ & \multicolumn{3}{|l|}{ phases } \\
\hline
\end{tabular}
present paper in comparison with previous results.

FIGURE 1. Influence the time of propagation $T_{\varepsilon}$

$\triangle$ Prospects. - A natural question is to investigate on the time interval $[0, T]$ with $T>0$ the asymptotic behavior of solutions to Euler equations when BULLETIN DE LA SOCIÉTÉ MATHÉMATIQUe DE FRANCE 
the initial data are as in (5.13). In [28], this problem is tackled by the usual BKW method relying on expansions of the form

$$
\boldsymbol{u}_{\infty}^{\varepsilon}(t, x):=\sum_{k=0}^{\infty} \varepsilon^{k} U_{k}\left(t, x, \varepsilon^{-1} \varphi_{0}(t, x)\right), \quad \partial_{\theta} U_{0}^{*} \not \equiv 0 .
$$

Modulation equations for the main profile $U_{0}$ are proposed. However these transport equations are not hyperbolic so that they are ill posed (in the sense of Hadamard) with respect to the initial value problem. It confirms that a BKW construction based on (5.14) is not relevant.

The contribution [28] does not explain why expansion (5.14) is not the good one. We come back below to this point. At first sight, Theorem 3.1 does not include large amplitude waves since $\boldsymbol{u}_{b}^{\varepsilon}-\boldsymbol{u}_{0}=O\left(\varepsilon^{1 / \ell}\right) \ll O(1)$. A change of variables leads to recant this impression. Suppose that $\boldsymbol{u}_{0} \equiv 0$ and $\partial_{\theta} U_{1}^{*} \not \equiv 0$. Then define

$$
\dot{\boldsymbol{u}}_{b}^{\varepsilon}(t, x):=\varepsilon^{-1 / \ell} \boldsymbol{u}_{b}^{\varepsilon}\left(\varepsilon^{-1 / \ell} t, x\right), \quad \dot{\boldsymbol{p}}_{b}^{\varepsilon}(t, x):=\varepsilon^{-2 / \ell} \boldsymbol{p}_{b}^{\varepsilon}\left(\varepsilon^{-1 / \ell} t, x\right) .
$$

Observe that the structure of $\dot{\boldsymbol{u}}_{b}^{\varepsilon}$ and $\dot{\boldsymbol{p}}_{b}^{\varepsilon}$ is very different from the one in (5.14) since we have

$$
\begin{aligned}
& \dot{\boldsymbol{u}}_{b}^{\varepsilon}(t, x)=\sum_{k=1}^{\infty} \varepsilon^{(k-1) / \ell} U_{k}\left(\varepsilon^{-1 / \ell} t, x, \varepsilon^{-1} \varphi_{g}^{\varepsilon}\left(\varepsilon^{-1 / \ell} t, x\right)\right)+\varepsilon^{-1 / \ell} \boldsymbol{c} \boldsymbol{u}_{b}^{\varepsilon}\left(\varepsilon^{-1 / \ell} t, x\right), \\
& \dot{\boldsymbol{p}}_{b}^{\varepsilon}(t, x)=\sum_{k=1}^{\infty} \varepsilon^{(k-2) / \ell} P_{k}\left(\varepsilon^{-1 / \ell} t, x, \varepsilon^{-1} \varphi_{g}^{\varepsilon}\left(\varepsilon^{-1 / \ell} t, x\right)\right)+\varepsilon^{-2 / \ell} \boldsymbol{c} \boldsymbol{p}_{b}^{\varepsilon}\left(\varepsilon^{-1 / \ell} t, x\right) .
\end{aligned}
$$

The functions $\dot{\boldsymbol{u}}_{b}^{\varepsilon}$ and $\dot{\boldsymbol{p}}_{b}^{\varepsilon}$ satisfy

$$
\partial_{t} \dot{\boldsymbol{u}}_{b}^{\varepsilon}+\left(\dot{\boldsymbol{u}}_{b}^{\varepsilon} \cdot \nabla\right) \dot{\boldsymbol{u}}_{b}^{\varepsilon}+\nabla \dot{\boldsymbol{p}}_{b}^{\varepsilon}=\dot{\boldsymbol{f}}_{b}^{\varepsilon}, \quad \operatorname{div} \dot{\boldsymbol{u}}_{b}^{\varepsilon}=0, \quad \dot{\boldsymbol{f}}_{b}^{\varepsilon}(t, x)=\varepsilon^{-2 / \ell} \boldsymbol{f}_{b}^{\varepsilon}\left(\varepsilon^{-1 / \ell} t, x\right) .
$$

The functions $\dot{\boldsymbol{u}}_{b}^{\varepsilon}$ are oscillations of the order 1 . They are approximate solutions of $(\mathcal{E})$ on the small interval $\left[0, \varepsilon^{1 / \ell} T\right]$. Indeed, for all $m \in \mathbb{N}$, the family $\left\{\dot{\boldsymbol{f}}_{b}^{\varepsilon}\right\}_{\varepsilon}$ is subjected to the uniform majoration

$$
\sup _{\left.\varepsilon \in] 0, \varepsilon_{0}\right]} \varepsilon^{-N / \ell+2 / \ell+d / 2+3+m}\left\|\dot{\boldsymbol{f}}_{b}^{\varepsilon}\right\|_{\mathcal{W}_{\varepsilon^{(1 / \ell)} T}^{m}}^{m}<\infty .
$$

If moreover $N=+\infty$ and

$$
\varphi_{1}(0, .) \equiv \cdots \equiv \varphi_{\ell-1}(0, .) \equiv 0, U_{k+1}(0, .) \equiv 0, \quad \forall k \in \mathbb{N} \backslash(\ell \mathbb{N}),
$$

the trace $\dot{\boldsymbol{u}}_{b}^{\varepsilon}(0,$.$) has the form$

$$
\dot{\boldsymbol{u}}_{b}^{\varepsilon}(0, x)=\sum_{k=0}^{\infty} \varepsilon^{k} U_{1+\ell k}\left(0, x, \varepsilon^{-1} \varphi_{0}(0, x)\right), \quad \partial_{\theta} U_{1}^{*} \not \equiv 0 .
$$

At the time $t=0$, we recover (5.14). Now the construction underlying the Theorem 3.1 reveals that in general

$$
\left.\left.\varphi_{k}(t, .) \not \equiv 0, \quad \forall t \in\right] 0, T\right], \quad \forall k \in\{2, \ldots, \ell-1\} .
$$

TOME $134-2006-\mathrm{N}^{\mathrm{O}} 1$ 
The functions $\varphi_{j}$ with $j \in\{2, \cdots, \ell-1\}$ are not present when $t=0$. But the description of $\dot{\boldsymbol{u}}_{b}^{\varepsilon}(t,$.$) on the interval \left[0, \varepsilon^{1-k / \ell} T\right]$ with $k \in\{2, \cdots, \ell-1\}$ requires the introduction of the phase shifts $\varphi_{j}$ for $j \in\{2, \cdots, k\}$.

The life span of $\dot{\boldsymbol{u}}_{b}^{\varepsilon}(t,$.$) is \varepsilon^{1 / \ell} T$. There are various manners to get a family $\left\{\dot{\boldsymbol{u}}_{b}^{\varepsilon}(t, .)\right\}_{\varepsilon \in] 0,1]}$ which is defined on some interval $[0, \widetilde{T}]$ with $\widetilde{T}>0$ independent on $\varepsilon$. In particular, we can:

- Select any $\widetilde{T}>0$ when $T=+\infty$. However nothing guarantees that the functions $\dot{\boldsymbol{u}}_{b}^{\varepsilon}$ are still approximate solutions on the interval $[0, \widetilde{T}]$. Indeed, since $t$ is replaced by $\varepsilon^{-1 / \ell} t$, the size of the error terms $\dot{\boldsymbol{f}}_{b}^{\varepsilon}$ depends on the increase of $\boldsymbol{f}_{b}^{\varepsilon}$ with respect to $t$. At this level, we are faced with secular growth problems [23].

- Make $\ell$ goes to $\infty$. When performing the formal analysis, arbitrary values can be given to the parameters $\varepsilon \in] 0,1]$ and $\ell \in \mathbb{N}_{*}$. For instance $\varepsilon$ can be fixed whereas $\ell$ goes to $\infty$. Or $\ell=-(\ln \varepsilon) /(\ln 2)$ so that $\varepsilon^{1 / \ell} T=\frac{1}{2} T \geq \widetilde{T}>0$. Even at a formal level, difficulties occur in order to justify some convergence process. At any rate, the description of $\dot{\boldsymbol{u}}_{b}^{\varepsilon}(t,$.$) on the whole interval [0, \widetilde{T}]$ needs the introduction of an infinite cascade of phases $\left\{\varphi_{j}\right\}_{j \in \mathbb{N}_{*}}$. It is like if, as you come closer to $\widetilde{T}$, you need more and more precision on the small scales of the solution.

Such a phenomenon does not occur when constructing large amplitude oscillations for systems of conservation laws in one space dimension [11], [15]. It is specific to the multidimensional framework and it is deeply linked with the incompressible constraint. It explains why the classical approach of [28] fails. Theorem 3.1 shows that one must give up one phase expansions and instead accept the idea that new phases are generated.

Two reasons could explain why this remark has not yet been made:

- The creation of the phases $\varphi_{j}$ is not due to well-known mechanisms. It is not linked with resonances. It is related neither to dispersive nor to diffractive effects.

- The most simple constructions indicating the persistence of oscillations, based on shear layers (1.1), involve only the phase $\varphi_{0}$. In fact, expressions like $\boldsymbol{u}_{s}^{\varepsilon}$ have a very special form. Let us explain why. Change the variable $t$ into $\varepsilon^{1 / \ell} t$ and $\boldsymbol{u}_{s}^{\varepsilon}$ into $\dot{\boldsymbol{u}}_{s}^{\varepsilon}:=\varepsilon^{1 / \ell} \boldsymbol{u}_{s}^{\varepsilon}$. The main phase $\varphi_{0}(t, x) \equiv x_{2}$ remains the same. Now we are faced with

$$
\dot{\boldsymbol{u}}_{s}^{\varepsilon}(t, x):={ }^{t}\left(\varepsilon^{1 / \ell} \boldsymbol{g}\left(x_{2}, \varepsilon^{-1} x_{2}\right), 0, \varepsilon^{1 / \ell} \boldsymbol{h}\left(x_{1}-\varepsilon^{1 / \ell} \boldsymbol{g}\left(x_{2}, \varepsilon^{-1} x_{2}\right) t, x_{2}, \varepsilon^{-1} x_{2}\right)\right) .
$$

It is still a solution of Euler equations. Now it falls in the framework of the Theorem 3.1. The constraints on $\bar{U}_{2}={ }^{t}\left(\bar{U}_{2}^{1}, \bar{U}_{2}^{2}, \bar{U}_{2}^{3}\right)$

$$
\bar{U}_{2}^{1} \equiv \bar{U}_{2}^{2} \equiv 0, \quad \partial_{t} \bar{U}_{2}^{3}+\left\langle\boldsymbol{g} \partial_{1} \boldsymbol{h}\right\rangle=0
$$

BULletin DE LA SOCiÉtÉ MATHÉmATiQUE DE FRANCE 
The contribution $\bar{U}_{2}$ is non trivial but it is polarized so that $\bar{U}_{2} \cdot \nabla \varphi_{0} \equiv 0$. Therefore it does not produce the phase shift $\varphi_{2}$. The same phenomenon occurs concerning $\varphi_{3}, \cdots, \varphi_{\ell-1}$. These terms are not present. It turns out that the expansion $\boldsymbol{u}_{s}^{\varepsilon}$ involves only the phase $\varphi_{0}(t, x) \equiv x_{2}$.

5.6. Some heuristical interpretation. - Turbulence and intermittency are topics which represent extremely different points of view. Two approaches compete:

a) The deterministic approach which studies the time evolution of flows arising in fluid mechanics [1], [4], [12], [14], [26].

b) The statistical approach in which the velocity of the fluid is a random variable [16], [24].

Attempts have been made in order to bring together the fields a) and b), see for instance [13]. Something in this direction can also be made with the help of the results 3.1 .

Theorem 3.1 is mainly connected with a). It brings various informations related to the propagation of quasi-singularities. These aspects have been detailed in the preceding paragraphs. In this subsection 5.6, we briefly explain b) and we draw (in the setting of Theorem 3.1) a phenomenological comparison between a) and b).

The statistical approach. - It deals with quantitative informations.

These informations are obtained at the level of expressions, say $\mu(x)$, which in general do not depend on the time $t$. The introduction of $\mu$ can be achieved by looking at stationary statistical solutions [16] of the Navier-Stokes equations that is

$$
\mu(x) \equiv \lim _{T \rightarrow \infty} \frac{1}{T} \int_{0}^{T} \boldsymbol{u}(t, x) \mathrm{d} t
$$

or in conjunction with the ensemble average operator (see [24], V-6) marked by the brackets $\langle$.$\rangle . We follow this second option.$

The description below is extracted from the book of M. Lesieur [24] (chapters $\mathrm{V}$ and VI). Work in dimension $d=3$. Interesting quantities are the mean kinetic energy

$$
\frac{1}{2}\left\langle\mu(x)^{2}\right\rangle \sim \int_{\mathbb{R}^{3}}|\mu(x)|^{2} \mathrm{~d} x,
$$

the enstrophy (that is the space integral of the square norm of the vorticity)

$$
\frac{1}{2}\left\langle\omega(x)^{2}\right\rangle \sim \int_{\mathbb{R}^{3}}|\omega(x)|^{2} \mathrm{~d} x, \quad \omega(x):=\nabla \wedge \mu(x),
$$

and the rate of dissipation $e \sim \kappa\left\langle\omega(x)^{2}\right\rangle$.

In the setting of isotropic turbulence, these three quantities can be expressed in terms of some scalar function $k \mapsto E(k)$. The real number $E(k)$ represents the density of kinetic energy at wave number $k$ (or the kinetic energy in Fourier space integrated on a sphere of radius $k$ ).

TOME $134-2006-\mathrm{N}^{\mathrm{O}} 1$ 
The relevant relations are the following:

[24, V-10-4]: $\quad \frac{1}{2}\left\langle\mu(x)^{2}\right\rangle=\int_{0}^{+\infty} E(k) \mathrm{d} k$.

[24, V-10-15]: $\quad \frac{1}{2}\left\langle\omega(x)^{2}\right\rangle=\int_{0}^{+\infty} k^{2} E(k) \mathrm{d} k$.

[24, VI-3-15]: $\quad e=2 \kappa \int_{0}^{+\infty} k^{2} E(k) \mathrm{d} k$.

Kolmogorov's theory assumes that

$$
\text { [24, VI-4-1]: } \exists c>0, E(k)=c \mathrm{e}^{\frac{2}{3}} k^{-\frac{5}{3}}, \forall k \in\left[k_{i}, k_{d}\right] \text {. }
$$

This law is valid up to the frequency $k_{d}$ with

$$
[24, \mathrm{VI}-4-2]: \quad k_{d} \sim\left(\mathrm{e} / \kappa^{3}\right)^{\frac{1}{4}} .
$$

The small quantity $\varepsilon:=k_{d}^{-1}$ is the Kolmogorov dissipative scale. Relations [24, VI-3-15] and [24, VI-4-2] imply that the rate of injection of kinetic energy $e$ is linked to the number $\ell$ according to $e \sim \varepsilon^{-1+3 / \ell}$. We recover here that $e \sim 1$ when $\ell=3$ (see [4]).

A starting point for the conventional theory of turbulence is the notion that, on average, kinetic energy is transfered from low wave numbers modes to high wave numbers modes. A recent paper [16] put forward the following idea: in the spectral region below that of injection of energy, an inverse (from high to low modes) transfer of energy takes place. At any rate, it is a central question to determine how the kinetic energy is distributed.

Phenomenological comparison. - The statistical approach is concerned with the spectral properties of solutions. Below, we draw a parallel with the propagation of quasi-singularities as it is described in Theorem 3.1.

Suppose (5.15) and consider $\dot{\boldsymbol{u}}_{b}^{\varepsilon}$. Let us examine how the square $\mathcal{F}\left(\dot{\boldsymbol{u}}_{b}^{\varepsilon}\right)(t, \xi)^{2}$ of the Fourier transform of $\dot{\boldsymbol{u}}_{b}^{\varepsilon}(t, x)$ is distributed. To this end, consider the application

$$
\begin{gathered}
\widetilde{E}(t, .): \mathbb{R}^{+} \longrightarrow \mathbb{R}^{+}, \\
k \longmapsto \widetilde{E}(t, k):=\int_{\left\{\xi \in \mathbb{R}^{d} ;|\xi|=k\right\}}\left|\mathcal{F}\left(\dot{\boldsymbol{u}}_{b}^{\varepsilon}\right)(t, \xi)\right|^{2} \mathrm{~d} \sigma(\xi) .
\end{gathered}
$$

The initial data $\dot{\boldsymbol{u}}_{b}^{\varepsilon}(0,$.$) has a spectral gap. In another words, the graph of$ the function $k \mapsto \widetilde{E}(0, k)$ appears concentrated around the two characteristic wave numbers $k \simeq 1$ and $k \simeq \varepsilon^{-1}=k_{d}$. In view of (5.16), this situation does not persist. At the time $t=\varepsilon^{1 / \ell}$, the concentration is around $\ell$ characteristic wave numbers which are intermediate between the two preceding ones. This corresponds to a discrete cascade of energy.

To describe the fluid on the time interval $[0, \widetilde{T}]$ with $\widetilde{T}>0$, we need to introduce an infinite cascade of phase shifts. The intuition ${ }^{(1)}$ is that the graph of $\widetilde{E}$ becomes continuous (no more gap). This corresponds to the impression

(1) Even at a formal level, difficulties occur in order to justify the different convergences. Rigorous results in this direction seem to be a difficult task. 
of an infinite cascade of energy. This remark is consistent with engineering experiments and the observations reported in the statistical approach.

The turbulent phenomena which we study are very complex in their realization. When $t>0$, the description of $\dot{\boldsymbol{u}}_{b}^{\varepsilon}(t,$.$) involves an infinite set of phases$ so that computations and representations are hard to implement. It gives the impression of a chaos. Nevertheless, our analysis reveals that these phenomena contain no mystery in their generation. On the contrary quantitative and qualitative features can be predicted in the framework of non linear geometric optics.

\section{About Navier-Stokes type equations}

Many real models involve dissipation terms which vanish or are small in some directions. It follows that hyperbolic features can still take place. In such cases, the form of the singularities and the structure of the viscosity are deeply linked. This general principle is the basis of the article [7] which is related to compressible Euler equations. Our aim in this section is to perform a similar analysis in the setting of incompressible equations.

More precisely, we consider some Navier-Stokes equation with vanishing anisotropic viscosity. We work with the variables $t, x$ and $\theta$. The introduction of the (periodic) fast variable $\theta$ induces new difficulties when constructing approximate solutions $u_{b}^{\varepsilon}(t, x, \theta)$. First, because the parabolic perturbation must be formulated in $(x, \theta)$. Secondly, because we have to solve the divergence free relation in $(x, \theta)$, that is

$$
\varepsilon \operatorname{div} u_{b}^{\varepsilon}(t, x, \theta)+\nabla \varphi_{b}^{\varepsilon}(t, x) \cdot \partial_{\theta} u_{b}^{\varepsilon}(t, x, \theta)=0 .
$$

Yet, the introduction of $\theta$ is needed to progress. The distinction between slow and fast variables is crucial when studying the stability. Indeed, we will have to extract mean values.

6.1. Approximate solutions. - In practice, the dissipation terms are often measured through experiments. They are given data and the matter is to adapt the quasi-singularities to the parabolic perturbation.

Below, we follow the opposite way. We fix some oscillation $\left\{\widetilde{\boldsymbol{u}}_{b}^{\varepsilon}\right\}_{\varepsilon}$ as in (3.12) and we adjust the dissipation terms accordingly. We require that the BKW calculus of Section 4 is not modified, except possibly for the introduction of well-known source terms. Then, we say that the viscosity is compatible with the complete phase $\varphi_{b}^{\varepsilon}$.

These two approaches amount ultimately to the same thing. Note however that the second one is much easier. Indeed, it is difficult to obtain quasisingularities with a prescribed stratification given by some $\varphi_{b}^{\varepsilon}$.

TOME $134-2006-\mathrm{N}^{\mathrm{O}} 1$ 
Real compatible viscosity. - Consider smooth coefficients $\mathfrak{q}_{\alpha \beta}^{i j}$ such that

$$
\left(\mathfrak{q}_{\alpha \beta}^{i j}(t, x)\right)_{1 \leq i, j \leq d} \in C^{\infty}\left([0, T] \times \mathbb{R}^{d} ; S^{d}\right), \quad \forall(\alpha, \beta) \in\{1, \ldots, d\}^{2} .
$$

By convention, the matrix symbol

$$
\mathfrak{q}(t, x, \xi):=\left(\sum_{1 \leq i, j \leq d} \mathfrak{q}_{\alpha \beta}^{i j}(t, x) \xi_{i} \xi_{j}\right)_{1 \leq \alpha, \beta \leq d}
$$

is in the (elliptic) class $E_{+}^{d}$ if

$$
\sum_{1 \leq \alpha, \beta, i, j \leq d} \mathfrak{q}_{\alpha \beta}^{i j}(t, x) \xi_{i} \xi_{j} u^{\alpha} u^{\beta} \geq 0, \quad \forall(t, x, \xi, u) \in[0, T] \times\left(\mathbb{R}^{d}\right)^{3} .
$$

The expression $\widetilde{\boldsymbol{u}}_{b}^{\varepsilon}$ undergoes rapid variations in the direction

$$
\mathfrak{X}_{b 1}^{\varepsilon}(t, x):=\left|X_{b}^{\varepsilon}(t, x)\right|^{-1} X_{b}^{\varepsilon}(t, x), \quad X_{b}^{\varepsilon}(t, x)=\nabla \varphi_{b}^{\varepsilon}(t, x) .
$$

Complete the unit vector $\mathfrak{X}_{b 1}^{\varepsilon}(t, x)$ into some orthonormal basis of $\mathbb{R}^{d}$

$$
\mathfrak{X}_{b i}^{\varepsilon}(t, x) \cdot \mathfrak{X}_{b j}^{\varepsilon}(t, x)=\delta_{i j}, \quad \forall(i, j) \in\{1, \ldots, d\}^{2}
$$

so that all the vector fields $\mathfrak{X}_{b i}^{\varepsilon}$ are smooth functions on $[0, T] \times \mathbb{R}^{d}$. The corresponding differential operators are denoted

$$
\mathfrak{X}_{b i}^{\varepsilon}\left(\partial_{x}\right):=\mathfrak{X}_{b i}^{\varepsilon}(t, x) \cdot \nabla, \quad i \in\{1, \ldots, d\} .
$$

Their adjoints are

$$
\mathfrak{X}_{b i}^{\varepsilon}\left(\partial_{x}\right)^{*}:=\mathfrak{X}_{b i}^{\varepsilon}(t, x) \cdot \nabla+\operatorname{div}\left(\mathfrak{X}_{b i}^{\varepsilon}\right)(t, x), \quad i \in\{1, \ldots, d\} .
$$

In order to be compatible with the propagation of oscillations, the viscosity must be small enough (of size $\varepsilon^{2}$ or less) in the direction $\mathfrak{X}_{b 1}^{\varepsilon}$. It can be of size $\varepsilon^{0}$ or less in the other directions. Let $\mathfrak{q} \in E_{+}^{d}$ and $m=\left(m_{1}, \ldots, m_{d}\right) \in \mathbb{N}^{d}$ a multi-indice such that

$$
m_{1} \geq 1, m_{2} \geq 0, \ldots, m_{d} \geq 0 .
$$

The preceding considerations lead to the following differential operator of order two

$$
\begin{aligned}
\mathcal{B}_{\mathfrak{q}}^{m}\left(\partial_{x}\right): C^{\infty}\left([0, T] \times \mathbb{R}^{d} ; \mathbb{R}^{d}\right) & \longrightarrow C^{\infty}\left([0, T] \times \mathbb{R}^{d} ; \mathbb{R}^{d}\right), \\
\boldsymbol{u}(t, x) & \longmapsto\left(\mathcal{B}_{\mathfrak{q}}^{m}\left(\partial_{x}\right) \boldsymbol{u}\right)(t, x)
\end{aligned}
$$

where

$$
\left(\mathcal{B}_{\mathfrak{q}}^{m}\left(\partial_{x}\right) \boldsymbol{u}\right)_{\alpha}(t, x):=\sum_{i=1}^{d} \varepsilon^{m_{i}} \mathfrak{X}_{b i}^{\varepsilon}\left(\partial_{x}\right)^{*}\left\{\sum_{j, \beta=1}^{d} \mathfrak{q}_{\alpha \beta}^{i j}(t, x) \varepsilon^{m_{j}} \mathfrak{X}_{b j}^{\varepsilon}\left(\partial_{x}\right) \boldsymbol{u}^{\beta}(t, x)\right\} .
$$

It turns out that the real viscosity $\mathcal{B}_{\mathfrak{q}}^{m}\left(\partial_{x}\right)$ is compatible with $\varphi_{b}^{\varepsilon}$.

BULLETIN DE LA SOCiÉTÉ MATHÉMATIQUe DE FRANCE 
Artificial compatible viscosity. — Introduce

$$
\begin{aligned}
& \mathfrak{X}_{b 1}^{\varepsilon}\left(\partial_{x, \theta}\right):=\mathfrak{X}_{b 1}^{\varepsilon}\left(\partial_{x}\right)+\varepsilon^{-1}\left|X_{b}^{\varepsilon}(t, x)\right| \times \partial_{\theta}, \\
& \mathfrak{X}_{b j}^{\varepsilon}\left(\partial_{x, \theta}\right):=\mathfrak{X}_{b j}^{\varepsilon}\left(\partial_{x}\right), \quad \forall j \in\{2, \ldots, d\} .
\end{aligned}
$$

The action of $\mathcal{B}_{\mathfrak{q}}^{m}\left(\partial_{x}\right)$ can be interpreted in $(x, \theta)$. It yields

$$
\begin{aligned}
\mathcal{B}_{\mathfrak{q}}^{m}\left(\partial_{x, \theta}\right): C^{\infty}\left([0, T] \times \mathbb{R}^{d} \times \mathbb{T} ; \mathbb{R}^{d}\right) & \longrightarrow C^{\infty}\left([0, T] \times \mathbb{R}^{d} \times \mathbb{T} ; \mathbb{R}^{d}\right), \\
\boldsymbol{u}(t, x, \theta) & \longmapsto\left(\mathcal{B}_{\mathfrak{q}}^{m}\left(\partial_{x, \theta}\right) \boldsymbol{u}\right)(t, x, \theta)
\end{aligned}
$$

where

$$
\begin{aligned}
& \left(\mathcal{B}_{\mathfrak{q}}^{m}\left(\partial_{x, \theta}\right) \boldsymbol{u}\right)_{\alpha}(t, x, \theta) \\
& \quad:=\sum_{i=1}^{d} \varepsilon^{m_{i}} \mathfrak{X}_{b i}^{\varepsilon}\left(\partial_{x, \theta}\right)^{*}\left\{\sum_{j, \beta=1}^{d} \mathfrak{q}_{\alpha \beta}^{i j}(t, x) \varepsilon^{m_{j}} \mathfrak{X}_{b j}^{\varepsilon}\left(\partial_{x, \theta}\right) \boldsymbol{u}^{\beta}(t, x, \theta)\right\} .
\end{aligned}
$$

One is faced here with some negative differential operator of the order two, where all the derivatives $\partial_{j}$ and $\partial_{\theta}$ have $\varepsilon^{0}$ or less $\left(\varepsilon^{k}\right.$ with $\left.k \geq 0\right)$ in factor. Thereafter, we will carry on the analysis with the prototype of such operators, that is the diagonal action given by

$$
\Delta:=\Delta_{x}+\partial_{\theta}^{2}, \quad \Delta_{x}:=\partial_{1}^{2}+\cdots+\partial_{d}^{2} .
$$

This Laplacian $\Delta$ is of course an artificial viscosity. In fact, throughout the Subsection 6.1 , the symbol $\Delta$ can be replaced by any of the compatible actions $\mathcal{B}_{\mathfrak{q}}^{m}$. It is used here only to simplify the presentation.

Statement of the result. - Fix some number $\nu \geq 0$. Select a smooth solution $\boldsymbol{u}_{0}(t, x) \in \mathcal{W}_{T}^{\infty}$ of

$$
\partial_{t} \boldsymbol{u}_{0}+\left(\boldsymbol{u}_{0} \cdot \nabla\right) \boldsymbol{u}_{0}+\nabla \boldsymbol{p}_{0}=\nu \Delta_{x} \boldsymbol{u}_{0}, \quad \operatorname{div} \boldsymbol{u}_{0}=0 .
$$

Choose a phase $\varphi_{0}(t, x) \in C^{1}\left([0, T] \times \mathbb{R}^{d}\right)$ with $\nabla \varphi_{0}(t, x) \in C_{b}^{\infty}\left([0, T] \times \mathbb{R}^{d}\right)$. Suppose that it satisfies the eiconal equation (3.3) and the condition (3.5). We need also a non degeneracy hypothesis on $\varphi_{0}$ which enables to prove that approximate solutions are exactly divergence free in the $(t, x, \theta)$ variables. We suppose that we can find a direction $\zeta \in \mathbb{R}^{d} \backslash\{0\}$ such that

$$
\exists c>0 ; \quad \nabla \varphi_{0}(t, x) \cdot \zeta \geq c, \quad \forall(t, x) \in[0, T] \times \mathbb{R}^{d} .
$$

Proposition 6.1. - The functions $\boldsymbol{u}_{0}$ and the phase $\varphi_{0}$ are as above. Choose any $b=(\ell, N) \in \mathbb{N}^{2}$ such that $0<\ell<N$. Select arbitrary data

$$
U_{k 0}^{*}(x, \theta) \in H^{\infty}, \quad\left\langle U_{k 0}\right\rangle(x) \in H^{\infty}, \quad \varphi_{k 0}(x) \in H^{\infty}, \quad 1 \leq k \leq N .
$$

The following preliminaries (i), (ii) and (iii) can be achieved.

(i) There are finite sequences $\left\{U_{k}\right\}_{1 \leq k \leq N}$ and $\left\{P_{k}\right\}_{1 \leq k \leq N}$ with

$$
U_{k}(t, x, \theta) \in \mathcal{W}_{T}^{\infty}, \quad P_{k}(t, x, \theta) \in \mathcal{W}_{T}^{\infty}, \quad 1 \leq k \leq N
$$

TOME $134-2006-\mathrm{N}^{\mathrm{O}} 1$ 
and which are such that

$$
\begin{gathered}
\Pi_{0}(0, x) U_{k}^{*}(0, x, \theta)=\Pi_{0}(0, x) U_{k 0}^{*}(x, \theta), \quad \forall k \in\{1, \ldots, N-\ell\}, \\
\left\langle U_{k}\right\rangle(0, x)=\left\langle U_{k 0}\right\rangle(x), \quad \forall k \in\{1, \ldots, N-\ell\} .
\end{gathered}
$$

(ii) There is a finite sequence $\left\{\varphi_{k}\right\}_{1 \leq k \leq N}$ with

$$
\varphi_{k}(t, x) \in \mathcal{W}_{T}^{\infty}, \quad \forall k \in\{1, \ldots, N\}
$$

and which is such that

$$
\varphi_{k}(0, x)=\varphi_{k 0}(x), \quad \forall k \in\{1, \ldots, N\} .
$$

(iii) There is $\left.\left.\varepsilon_{0} \in\right] 0,1\right]$ and correctors

$$
\left.\left.c u_{b}^{\varepsilon}(t, x, \theta) \in \mathcal{W}_{T}^{\infty}, \quad \varepsilon \in\right] 0, \varepsilon_{0}\right],
$$

which give rise to a family satisfying $\left\{c u_{b}^{\varepsilon}\right\}_{\varepsilon}=O\left(\varepsilon^{(N+1-\ell) / \ell}\right)$.

With the materials of (i), (ii) and (iii), construct $\varphi_{b}^{\varepsilon}$, the initial data

$$
h_{b}^{\varepsilon}(x, \theta):=\boldsymbol{u}_{0}(0, x)+\sum_{k=1}^{N} \varepsilon^{k / \ell} U_{k}(0, x, \theta)+c u_{b}^{\varepsilon}(0, x, \theta)
$$

and the functions $u_{b}^{\varepsilon}$ and $p_{b}^{\varepsilon}$ defined according to

$$
\left\{\begin{array}{l}
u_{b}^{\varepsilon}(t, x, \theta)=\boldsymbol{u}_{0}(t, x)+\sum_{k=1}^{N} \varepsilon^{k / \ell} U_{k}(t, x, \theta)+c u_{b}^{\varepsilon}(t, x, \theta), \\
p_{b}^{\varepsilon}(t, x, \theta)=\boldsymbol{p}_{0}(t, x)+\sum_{k=1}^{N} \varepsilon^{k / \ell} P_{k}(t, x, \theta) .
\end{array}\right.
$$

Then, all these expressions can be adjusted so that the functions $u_{b}^{\varepsilon}$ and $p_{b}^{\varepsilon}$ satisfy on the domain $[0, T] \times \mathbb{R}^{d} \times \mathbb{T}$ the following singular system

$$
\left\{\begin{aligned}
& \partial_{t} u_{b}^{\varepsilon}+\left(u_{b}^{\varepsilon} \cdot \nabla\right) u_{b}^{\varepsilon}+\nabla p_{b}^{\varepsilon}+ \varepsilon^{-1}\left(\partial_{t} \varphi_{b}^{\varepsilon}+u_{b}^{\varepsilon} \cdot \nabla \varphi_{b}^{\varepsilon}\right) \partial_{\theta} u_{b}^{\varepsilon} \\
&+\varepsilon^{-1} \partial_{\theta} p_{b}^{\varepsilon} \nabla \varphi_{b}^{\varepsilon}=\nu \Delta u_{b}^{\varepsilon}+f_{b}^{\varepsilon}, \\
& \operatorname{div} u_{b}^{\varepsilon}+\varepsilon^{-1} \nabla \varphi_{b}^{\varepsilon} \cdot \partial_{\theta} u_{b}^{\varepsilon}=0, \quad u_{b}^{\varepsilon}(0, x, \theta)=h_{b}^{\varepsilon}(x, \theta),
\end{aligned}\right.
$$

where the corrector $f_{b}^{\varepsilon}(t, x, \theta) \in \mathcal{W}_{T}^{\infty}$ satisfy $\left\{f_{b}^{\varepsilon}\right\}_{\varepsilon}=O\left(\varepsilon^{(N+2) / \ell-2}\right)$.

Define

$$
\mathfrak{g r a d} \mathfrak{d}_{b}^{\varepsilon}:=\varepsilon \nabla+X_{b}^{\varepsilon} \times \partial_{\theta}, \quad \mathfrak{d i v} v_{b}^{\varepsilon}:=\left(\mathfrak{g r a d} \mathfrak{d}_{b}^{\varepsilon}\right)^{\star}=\varepsilon \operatorname{div}+X_{b}^{\varepsilon} \cdot \partial_{\theta} .
$$

We need some material before proving Proposition 6.1. 
The divergence free relation in the variables $(t, x, \theta)$. - We can select some special right inverse of the application $\mathfrak{d i v}_{b}^{\varepsilon}: H_{T}^{\infty *} \longrightarrow H_{T}^{\infty *}$.

LEMMA 6.1. - There is a linear operator $\mathfrak{r i d i v}_{b}^{\varepsilon}: \operatorname{Im}\left(\mathfrak{d i v}_{b}^{\varepsilon}\right) \longrightarrow H_{T}^{\infty *}$ with

$$
\mathfrak{d i v}_{b}^{\varepsilon} \circ \mathfrak{r i d i v}_{b}^{\varepsilon} g=g, \quad \forall g \in \operatorname{Im}\left(\mathfrak{d i v} \mathfrak{v}_{b}^{\varepsilon}\right) .
$$

For all $m \in \mathbb{N}$, there is a constant $C_{m}>0$ such that

$$
\left\|\mathfrak{r i d i v} \mathfrak{b}_{b}^{\varepsilon} g\right\|_{H^{m}} \leq C_{m}\|g\|_{H^{m+1+\frac{1}{2} d}}, \quad \forall g \in \operatorname{Im}\left(\mathfrak{d i v}_{b}^{\varepsilon}\right) \text {. }
$$

Proof of Lemma 6.1. - Let $n \in \mathbb{N}_{*}$. Note

$$
t_{j}:=\frac{j T}{n}, \quad x_{j}=\frac{k}{n}, \quad 1 \leq j \leq n-1, k \in \mathbb{Z}^{d} .
$$

Consider a related partition of unity

$$
\begin{aligned}
& \chi_{(j, k)} \in C^{\infty}\left([0, T] \times \mathbb{R}^{d}\right), \quad(j, k) \in\{1, \ldots, n-1\} \times \mathbb{Z}^{d}, \\
& \sum_{j=1}^{n-1} \sum_{k \in \mathbb{Z}^{d}} \chi_{(j, k)}(t, x)=1, \quad \forall(t, x) \in[0, T] \times \mathbb{R}^{d}, \\
& \left\{(t, x) ; \chi_{(j, k)}(t, x) \neq 0\right\} \subset\left[t_{j}-\frac{2}{n}, t_{j}+\frac{2}{n}\right] \times B\left(x_{j}, \frac{2}{n}\right], \\
& \left\{(t, x) ; \chi_{(j, k)}(t, x)=1\right\} \supset\left[t_{j}-\frac{1}{n}, t_{j}+\frac{1}{n}\right] \times B\left(x_{j}, \frac{1}{n}\right] .
\end{aligned}
$$

By hypothesis, there is a function $v \in H_{T}^{\infty *} \operatorname{such}$ that $g=\mathfrak{d} \mathfrak{i v}_{b}^{\varepsilon} v$. Introduce

$$
v_{(j, k)}:=\chi_{(j, k)} v \in H_{T}^{\infty *}, \quad g_{(j, k)}:=\mathfrak{d i v} \mathfrak{v}_{b}^{\varepsilon} v_{(j, k)} .
$$

It suffices to exhibit ridiv $\operatorname{riv}_{b}^{\varepsilon} g_{(j, k)}$ and to show (6.5) with a constant $C_{m}$ which is uniform in $(j, k)$.

The problem of finding $\mathfrak{r i d i v}_{b}^{\varepsilon} g_{(j, k)}$ can be reduced to a model situation. This can be achieved by using a change of variables in $(t, x)$, based on (6.2). From now on, the time $t$ is viewed as a parameter, the space variable is $x=\left(x_{1}, \widehat{x}\right) \in$ $\mathbb{R} \times \mathbb{R}^{d-1}$, and we work with

$$
\begin{aligned}
& g=g^{*}=\mathfrak{d i v}_{b}^{\varepsilon} v=\left(\varepsilon \partial_{1}+\partial_{\theta}\right) v_{1}+\partial_{2} v_{2}+\cdots+\partial_{d} v_{d}, \\
& \{x ; g(x, \theta) \neq 0\} \subset\{x ; v(x, \theta) \neq 0\} \subset B\left(0, \frac{1}{2}\right] .
\end{aligned}
$$

Let $\psi \in C^{\infty}\left(\mathbb{R}^{d-1} ; \mathbb{R}^{+}\right)$be such that $\int_{\mathbb{R}^{d-1}} \psi(\widehat{x}) \mathrm{d} \widehat{x}=1$ and

$$
\{\hat{x} ; \psi(\widehat{x}) \neq 0\} \subset B(0,1], \quad\{\widehat{x} ; \psi(\widehat{x})=1\} \supset B\left(0, \frac{1}{2}\right] .
$$

Decompose $g$ according to

$$
g=(g-\breve{g}) \psi+\breve{g} \psi, \quad \breve{g}(x):=\int_{\mathbb{R}^{d-1}} g\left(x_{1}, \widehat{x}\right) \mathrm{d} \widehat{x}=\left(\varepsilon \partial_{1}+\partial_{\theta}\right) \breve{v}_{1} .
$$

Seek a special solution $u$ having the form

$$
u=\mathfrak{r i d i v}_{b}^{\varepsilon} g={ }^{t}(a, \operatorname{ridiv}[(g-\breve{g}) \psi]), \quad a \in H_{T}^{\infty *}
$$

TOME $134-2006-\mathrm{N}^{\mathrm{O}} 1$ 
where 'ridiv' is the operator of Lemma 4.1 applied in dimension $d-1$. It remains to control the scalar function $a$ which satisfies the constraint

$$
\varepsilon \partial_{1} a+\partial_{\theta} a=h:=\breve{g} \psi=\left(\varepsilon \partial_{1}+\partial_{\theta}\right)\left(\breve{v}_{1} \psi\right) .
$$

Take the explicit solution

$$
a\left(x_{1}, \widehat{x}, \theta\right)=\int_{-\infty}^{\theta} h\left(x_{1}+\varepsilon(s-\theta), \widehat{x}, s\right) \mathrm{d} s=\varepsilon^{-1} \int_{-\infty}^{0} h\left(x_{1}+r, \widehat{x}, \theta+\varepsilon^{-1} r\right) \mathrm{d} r .
$$

By construction

$$
a(x, \theta+1)=a(x, \theta), \quad \int_{\mathbb{T}} a(x, \theta) \mathrm{d} \theta=0, \quad \forall(x, \theta) \in \mathbb{R}^{d} \times \mathbb{T} .
$$

For $\left|x_{1}\right|+|\widehat{x}| \geq 2$, we find

$$
a(x, \theta)=\int_{-\infty}^{\theta} \frac{\mathrm{d}}{\mathrm{d} s}\left[\left(\breve{v}_{1} \psi\right)\left(x_{1}+\varepsilon(s-\theta), \widehat{x}, s\right)\right] \mathrm{d} s=\left(\breve{v}_{1} \psi\right)(x, \theta)=0 .
$$

It implies that

$$
\{(x, \theta) ; a(x, \theta) \neq 0\} \subset B(0 ; 2] .
$$

Note $\mathfrak{h}:=\partial_{\theta}^{-1} h \in H^{\infty *}$. Obviously

$$
\|\mathfrak{h}\|_{H^{m}} \leq C_{m}\|h\|_{H^{m}}, \quad \forall m \in \mathbb{N}, \quad\{(x, \theta) ; \mathfrak{h}(x, \theta) \neq 0\} \subset B(0 ; 1],
$$

and we have the identity

$$
a\left(x_{1}, \hat{x}, \theta\right)=\mathfrak{h}\left(x_{1}, \widehat{x}, \theta\right)-\int_{-x_{1}-1}^{-x_{1}+1} \partial_{1} \mathfrak{h}\left(x_{1}+r, \widehat{x}, \theta+\varepsilon^{-1} r\right) \mathrm{d} r .
$$

The term on the right is supported in $B(0,2]$. Use Fubini and Cauchy-Schwarz inequality to control the integration of $\partial_{1} \mathfrak{h}$. It yields $(6.5)$.

Proof of Proposition 6.1. - The BKW computations go as in Subsection 4.1. There are only minor modifications. Some of them are listed below. The equation (4.18) must be replaced by

$$
P_{\ell+1}^{*}=\ell \partial_{\theta}^{-1} W_{1}^{*}+\nu\left|X_{0}\right|^{-2} X_{0} \cdot \partial_{\theta}^{-1} \Delta W_{1}^{*} .
$$

The transport equations (4.19) becomes

$$
\begin{gathered}
\partial_{t} \bar{U}_{j+1}+\left(\boldsymbol{u}_{0} \cdot \nabla\right) \bar{U}_{j+1}+\left(\bar{U}_{j+1} \cdot \nabla\right) \boldsymbol{u}_{0}+\sum_{k=1}^{j}\left(\bar{U}_{k} \cdot \nabla\right) \bar{U}_{j+1-k} \\
+\sum_{k=1}^{j} \operatorname{div}\left\langle U_{k}^{*} \otimes U_{j+1-k}^{*}\right\rangle+\nabla \bar{P}_{j+1}=\nu \Delta_{x} \bar{U}_{j+1}, \\
\operatorname{div} \bar{U}_{j+1}=0,
\end{gathered}
$$

whereas (4.21) is modified into

$$
\partial_{t} W_{j+1}^{*}+\left(\boldsymbol{u}_{0} \cdot \nabla\right) W_{j+1}^{*}=M W_{j+1}^{*}+\nu \Pi_{0} \Delta W_{j+1}^{*}+f .
$$

BULLETIN DE LA SOCIÉTÉ MATHÉMATIQUE DE FRANCE 
where $f$ is known. Define

$$
\begin{aligned}
& \widetilde{u}_{b}^{\varepsilon}(t, x, \theta)=\boldsymbol{u}_{0}(t, x)+\sum_{k=1}^{N} \varepsilon^{k / \ell} U_{k}(t, x, \theta), \\
& \widetilde{p}_{b}^{\varepsilon}(t, x, \theta)=\boldsymbol{p}_{0}(t, x)+\sum_{k=1}^{N} \varepsilon^{k / \ell} P_{k}(t, x, \theta) .
\end{aligned}
$$

By construction, we have

$$
\begin{gathered}
\partial_{t} \widetilde{u}_{b}^{\varepsilon}+\left(\widetilde{u}_{b}^{\varepsilon} \cdot \nabla\right) \widetilde{u}_{b}^{\varepsilon}+\nabla \widetilde{p}_{b}^{\varepsilon}+\varepsilon^{-1}\left(\partial_{t} \varphi_{b}^{\varepsilon}+\widetilde{u}_{b}^{\varepsilon} \cdot \nabla \varphi_{b}^{\varepsilon}\right) \partial_{\theta} \widetilde{u}_{b}^{\varepsilon} \\
+\varepsilon^{-1} \partial_{\theta} \widetilde{p}_{b}^{\varepsilon} \nabla \varphi_{b}^{\varepsilon}=\nu \Delta \widetilde{u}_{b}^{\varepsilon}+\widetilde{f}_{b}^{\varepsilon}, \\
\operatorname{div} \widetilde{u}_{b}^{\varepsilon}+\varepsilon^{-1} \nabla \varphi_{b}^{\varepsilon} \cdot \partial_{\theta} \widetilde{u}_{b}^{\varepsilon}=\widetilde{g}_{b}^{\varepsilon}, \quad \widetilde{u}_{b}^{\varepsilon}(0, x, \theta)=\widetilde{h}_{b}^{\varepsilon}(x, \theta),
\end{gathered}
$$

where the correctors $\tilde{f}_{b}^{\varepsilon}$ and $\widetilde{g}_{b}^{\varepsilon}$ satisfy

$$
\left\{\tilde{f}_{b}^{\varepsilon}\right\}_{\varepsilon}=O\left(\varepsilon^{(N+1-\ell) / \ell}\right), \quad\left\{\widetilde{g}_{b}^{\varepsilon}\right\}_{\varepsilon}=O\left(\varepsilon^{(N+1-\ell) / \ell}\right) .
$$

It remains to absorb the term $\widetilde{g}_{b}^{\varepsilon}$. Use the decomposition

$$
g_{b}^{\varepsilon}=\left\langle\widetilde{g}_{b}^{\varepsilon}\right\rangle+\widetilde{g}_{b}^{\varepsilon *}, \quad\left\langle\widetilde{g}_{b}^{\varepsilon}\right\rangle \in \operatorname{Im}(\operatorname{div}), \quad \widetilde{g}_{b}^{\varepsilon *} \in \operatorname{Im}\left(\mathfrak{d i v} \mathfrak{v}_{b}^{\varepsilon}\right) .
$$

It suffices to choose

$$
c u_{b}^{\varepsilon}:=-\operatorname{ridiv}\left\langle\widetilde{g}_{b}^{\varepsilon}\right\rangle-\mathfrak{r i d i v}_{b}^{\varepsilon} \widetilde{g}_{b}^{* *}=O\left(\varepsilon^{(N+1-\ell) / \ell}\right) .
$$

6.2. A preliminary result of stability. - Consider the Cauchy problem

$$
\left\{\begin{array}{l}
\partial_{t} \boldsymbol{u}^{\varepsilon}+\left(\boldsymbol{u}^{\varepsilon} \cdot \nabla\right) \boldsymbol{u}^{\varepsilon}+\nabla \boldsymbol{p}^{\varepsilon}=\mathcal{B}_{\mathfrak{q}}^{m}\left(\partial_{x}\right) \boldsymbol{u}^{\varepsilon}, \quad \operatorname{div} \boldsymbol{u}^{\varepsilon}=0, \\
\boldsymbol{u}^{\varepsilon}(0, x)=u_{b}^{\varepsilon}\left(0, x, \varepsilon^{-1} \varphi_{b}^{\varepsilon}(0, x)\right),
\end{array}\right.
$$

where $u_{b}^{\varepsilon}(t, x, \theta)$ and $\varphi_{b}^{\varepsilon}(t, x)$ are given by the Proposition 6.1. Let $T_{\varepsilon}$ be the upper bound of the $T \geq 0$ such that (6.6) has a solution $\boldsymbol{u}^{\varepsilon} \in \mathcal{W}_{T}^{0}$. Classical results for fluid equations imply that $T_{\varepsilon}>0$. The matter is to investigate the singular limit ' $\varepsilon$ goes to zero'.

Generalities. - The analysis must contain the two following parts.

$\triangleright$ An existence result for a time $T_{0}$ which is independent on the small parameter $\left.\varepsilon \in] 0, \varepsilon_{0}\right]$. It is required that

$$
\left.\left.\inf \left\{T_{\varepsilon} ; \varepsilon \in\right] 0,1\right]\right\} \geq T_{0}>0 .
$$

Look at the special choice

$$
\mathfrak{q}_{\alpha \beta}^{i j}(t, x)=\nu \delta_{i j} \delta_{\alpha \beta}, \quad m_{1}=m_{2}=\cdots=m_{d}=1
$$

which corresponds to

$$
\mathcal{B}_{\mathfrak{q}}^{m}\left(\partial_{x}\right) \boldsymbol{u}^{\varepsilon}=\nu \varepsilon^{2} \Delta_{x} \boldsymbol{u}^{\varepsilon}
$$

When $\nu>0$, or when $\nu=0$ and $d=2$, we know [5], [25] that $T_{\varepsilon}=+\infty$ so that $T_{0}=+\infty$. When $\nu=0$ and $d \geq 3$, nothing guarantees that $T_{0}>0$. To our knowledge, this is an open question.

TOME $134-2006-\mathrm{N}^{\mathrm{O}} 1$ 
$\triangleright$ A convergence result. Since the error term $f_{b}^{\varepsilon}$ is small, we expect that the exact solution $\boldsymbol{u}^{\varepsilon}$ remains close to the approximate solution

$$
\boldsymbol{u}_{a}^{\varepsilon}(t, x):=u_{b}^{\varepsilon}\left(t, x, \varepsilon^{-1} \varphi_{b}^{\varepsilon}(t, x)\right) .
$$

This is verified on small time intervals. However, on the whole interval $\left[0, T_{0}\right]$, it is a delicate question to prove estimates on the difference $\boldsymbol{u}^{\varepsilon}-\boldsymbol{u}_{a}^{\varepsilon}$. In particular, we wonder if

$$
\lim _{\varepsilon \rightarrow 0}\left\|\varepsilon^{-1 / \ell}\left(\boldsymbol{u}^{\varepsilon}-\boldsymbol{u}_{a}^{\varepsilon}\right)\right\|_{L_{T_{0}}^{2}}=0 .
$$

Even if $f_{b}^{\varepsilon}=O\left(\varepsilon^{\infty}\right)$, the identity (6.7) is not sure to be true. Various amplification mechanisms can occur. Obvious instabilities are the phenomena which are detected by the 'monophase' description of Section 4 . They are not the only one. Other mechanisms can happen. By contrast, they are called hidden instabilities.

Examples of hidden instabilities can be found in the hyperbolic situation or when the influence of $\mathcal{B}_{\mathfrak{q}}^{m}$ is negligible. In [8], we work with the case $\mathcal{B}_{\mathfrak{q}}^{m} \equiv 0$ and $\ell=2$. Let us recall what can happen. Select a second (main) phase $\psi_{0}(t, x) \in \mathcal{W}_{T}^{\infty}$ such that

$$
\partial_{t} \psi_{0}+\left(\boldsymbol{u}_{0} \cdot \nabla\right) \psi_{0}=0, \quad \nabla \psi_{0} \wedge \nabla \varphi_{0} \not \equiv 0
$$

and disturb the Cauchy data of (6.6) according to

$$
\boldsymbol{u}^{\varepsilon}(0, x)=u_{b}^{\varepsilon}\left(0, x, \varepsilon^{-1} \varphi_{b}^{\varepsilon}(0, x)\right)+\varepsilon^{M / \ell} U\left(x, \varepsilon^{-1} \psi_{0}(0, x)\right), \quad M \gg \ell .
$$

The small oscillations contained in the perturbation of size $\varepsilon^{M / \ell}$ are not always kept under control. They interact with $\boldsymbol{u}_{a}^{\varepsilon}$ and with themselves. They can be organized in such a way to affect the leading oscillation $\boldsymbol{u}_{a}^{\varepsilon}$. Concretely (see [8]), we can adjust $U$ and $\psi_{0}$ so that there is a constant $C>0$ and times $\left.t_{\varepsilon} \in\right] 0, T_{\varepsilon}$ [ going to zero with $\varepsilon$ such that

$$
\left.\left.\left\|\left(\boldsymbol{u}^{\varepsilon}-\boldsymbol{u}_{a}^{\varepsilon}\right)\left(t_{\varepsilon}, .\right)\right\|_{L^{2}\left(\mathbb{R}^{d}\right)} \geq C \varepsilon^{\frac{1}{2}}, \quad \forall \varepsilon \in\right] 0, \varepsilon_{0}\right] .
$$

The power $\varepsilon^{M / \ell}$ at the time $t=0$ is turned into $\varepsilon^{\frac{1}{2}}$ at the time $t=t_{\varepsilon}$. In fact, such amplifications occur whatever the selection of $\ell \geq 2$. They imply minorations similar to (5.5) though the underlying mechanisms are completely distinct.

The first information brought by our BKW construction is that mean values $\bar{U}_{k}$ and oscillations $U_{k}^{*}$ do not play the same part. This fact is well illustrated by the rules of transformation (5.10). It means that we have to distinguish these quantities if we want to go further in the analysis.

This distinction can be done by involving the variables $(t, x, \theta)$ that is by working at the level of $(\mathcal{N S})$. To deal with $\left(u^{\varepsilon}, p^{\varepsilon}\right)(t, x, \theta)$ instead of $\left(\boldsymbol{u}^{\varepsilon}, \boldsymbol{p}^{\varepsilon}\right)(t, x)$ is usual in non linear geometric optics [27]. It allows to mark the terms apt to induce instabilities. It gives rise to new technical difficulties. For instance, we have to implement:

BULLETIN DE LA SOCIÉtÉ MATHÉMATIQUE DE FRANCE 
The Leray projector interpreted in the variables $(t, x, \theta)$. — Define

$$
\mathfrak{d}_{j, \varepsilon}:=\varepsilon \partial_{j}+\partial_{j} \varphi_{b}^{\varepsilon} \times \partial_{\theta}, \quad j \in\{0, \ldots, d\} .
$$

Introduce the closed subspace

$$
\mathrm{F}_{b}^{\varepsilon}:=\left\{u^{*} \in L_{T}^{2 *} ; \mathfrak{d i v} \mathfrak{v}_{b}^{\varepsilon} u^{*}=0\right\} \subset L_{T}^{2 *} .
$$

Note $\mathfrak{P}_{b}^{\varepsilon}$ the orthogonal projector from $L_{T}^{2 *}$ onto $\mathrm{F}_{b}^{\varepsilon}$. This is a self-adjoint operator such that

$$
\operatorname{ker} \mathfrak{d i v} v_{b}^{\varepsilon}=\operatorname{Im} \mathfrak{P}_{b}^{\varepsilon}, \quad \operatorname{Im} \mathfrak{g r a d} \mathfrak{d}_{b}^{\varepsilon}=\left(\operatorname{ker} \mathfrak{d} \mathfrak{i v}_{b}^{\varepsilon}\right)^{\perp}=\operatorname{ker} \mathfrak{P}_{b}^{\varepsilon} .
$$

Expand the function $u^{*} \in L_{T}^{2 *}$ in Fourier series and decompose the action of $\mathfrak{P}_{b}^{\varepsilon}$ in view of the Fourier modes

$$
u^{*}(t, x, \theta)=\sum_{k \in \mathbb{Z}_{*}} \boldsymbol{u}_{k}(t, x) \mathrm{e}^{i k \theta}, \quad \mathfrak{P}_{b}^{\varepsilon} u^{*}=\sum_{k \in \mathbb{Z}_{*}} \mathfrak{P}_{b k}^{\varepsilon} \boldsymbol{u}_{k}(t, x) \mathrm{e}^{i k \theta} .
$$

Simple computations indicate that

$$
\mathfrak{P}_{b k}^{\varepsilon} \boldsymbol{u}_{k}:=\mathrm{e}^{-i \varepsilon^{-1} k \varphi_{b}^{\varepsilon}} \Pi\left(D_{x}\right)\left(\mathrm{e}^{i \varepsilon^{-1} k \varphi_{b}^{\varepsilon}} \boldsymbol{u}_{k}\right) .
$$

The following result explains why the projector $\mathfrak{P}_{b}^{\varepsilon}$ is replaced by $\Pi_{0}$ when performing the BKW calculus.

LEMMA 6.2

(i) The family $\left\{\mathfrak{P}_{b}^{\varepsilon}\right\}_{\varepsilon}$ is in $\mathfrak{U L}^{0}$. We have $\left[\partial_{\theta} ; \mathfrak{P}_{b}^{\varepsilon}\right]=0$ and

$$
\left[\mathfrak{d}_{j, \varepsilon} ; \mathfrak{P}_{b}^{\varepsilon}\right]=0, \quad \forall j \in\{0, \ldots, d\} .
$$

(ii) The projector $\Pi_{b}^{\varepsilon}(t, x)$ is an approximation of $\mathfrak{P}_{b}^{\varepsilon}$ in the sense that

$$
\left\{\mathfrak{P}_{b}^{\varepsilon}-\Pi_{b}^{\varepsilon}\right\}_{\varepsilon} \in \varepsilon \mathfrak{U} \mathfrak{L}^{2+\frac{1}{2} d}, \quad\left\{\mathfrak{P}_{b}^{\varepsilon}\left(\mathrm{Id}-\Pi_{b}^{\varepsilon}\right)\right\}_{\varepsilon} \in \varepsilon \mathfrak{U} \mathfrak{L}^{1} .
$$

Proof of Lemma 6.2. - Since $\mathfrak{P}_{b}^{\varepsilon}$ is a projector in $L^{2}$, we are sure that it is bounded

$$
\left.\left.\left\|\mathfrak{P}_{b}^{\varepsilon} u\right\|_{L_{T}^{2}} \leq\|u\|_{L_{T}^{2}}, \quad \forall(\varepsilon, u) \in\right] 0, \varepsilon_{0}\right] \times L_{T}^{2} .
$$

It shows that $\left\{\mathfrak{P}_{b}^{\varepsilon}\right\}_{\varepsilon} \in \mathfrak{U} \mathfrak{L}^{0}$.

$$
\left[\mathfrak{d}_{j, \varepsilon} ; \mathfrak{P}_{b}^{\varepsilon}\right] u^{*}(t, x, \theta)=\sum_{k \in \mathbb{Z}_{*}}\left[\varepsilon \partial_{j}+i k \partial_{j} \varphi_{b}^{\varepsilon} ; \mathfrak{P}_{b k}^{\varepsilon}\right] \boldsymbol{u}_{k}(t, x) \mathrm{e}^{i k \theta}
$$

Observe that

$$
\begin{aligned}
\left(\varepsilon \partial_{j}+i k \partial_{j} \varphi_{b}^{\varepsilon}\right) \mathfrak{P}_{b k}^{\varepsilon} \boldsymbol{u}_{k} & =\mathrm{e}^{-i \varepsilon^{-1} k \varphi_{b}^{\varepsilon}} \Pi\left(D_{x}\right) \varepsilon \partial_{j}\left(\mathrm{e}^{i \varepsilon^{-1} k \varphi_{b}^{\varepsilon}} \boldsymbol{u}_{k}\right) \\
& =\mathfrak{P}_{b k}^{\varepsilon}\left(\varepsilon \partial_{j}+i k \partial_{j} \varphi_{b}^{\varepsilon}\right) \boldsymbol{u}_{k} .
\end{aligned}
$$

All these informations give access to the first assertion (i).

Now consider (ii). The asymptotic expansion formula for pseudodifferential operators say that for all $\boldsymbol{u}_{k}$ in $C_{0}^{\infty}\left(\mathbb{R}_{T}^{d}\right)$ we have

$$
\forall(t, x) \in \mathbb{R}_{T}^{d}, \quad \lim _{\varepsilon \rightarrow 0}\left\{\left(\mathfrak{P}_{b k}^{\varepsilon} \boldsymbol{u}_{k}\right)(t, x)-\Pi\left(\nabla \varphi_{b}^{\varepsilon}(t, x)\right) \boldsymbol{u}_{k}(t, x)\right\}=0 .
$$

TOME $134-2006-\mathrm{N}^{\mathrm{O}} 1$ 
Since $\Pi_{b}^{\varepsilon}=\Pi\left(\nabla \varphi_{b}^{\varepsilon}\right)$, it indicates that $\mathfrak{P}_{b}^{\varepsilon}$ is close to $\Pi_{b}^{\varepsilon}$. We have to make this information more precise. To this end, proceed to the decomposition

$$
u^{*}=v^{*}+\varepsilon \nabla p^{*}+\partial_{\theta} p^{*} \times X_{b}^{\varepsilon}, \quad v^{*}=\mathfrak{P}_{b}^{\varepsilon} u^{*} .
$$

We seek a solution $\left(v^{*}, p^{*}\right)$ of these constraints such that

$$
v^{*}=\Pi_{b}^{\varepsilon} u^{*}+\varepsilon \widetilde{v}^{*}, \quad p^{*}=\left\|X_{b}^{\varepsilon}\right\|^{-2} X_{b}^{\varepsilon} \cdot \partial_{\theta}^{-1} u^{*}+\varepsilon \widetilde{p}^{*} .
$$

After substitution, we find the relation

$$
-\nabla\left(\left\|X_{b}^{\varepsilon}\right\|^{-2} X_{b}^{\varepsilon} \cdot \partial_{\theta}^{-1} u^{*}\right)=\widetilde{v}^{*}+\varepsilon \nabla \widetilde{p}^{*}+\partial_{\theta} \widetilde{p}^{*} \times X_{b}^{\varepsilon}
$$

which must be completed by the condition

$$
-\operatorname{div}\left(\Pi_{b}^{\varepsilon} u^{*}\right)=\varepsilon \operatorname{div} \widetilde{v}^{*}+X_{b}^{\varepsilon} \cdot \partial_{\theta} \widetilde{v}^{*} .
$$

It follows that

$$
\widetilde{v}^{*}=-\mathfrak{P}_{b}^{\varepsilon}\left[\nabla\left(\left\|X_{b}^{\varepsilon}\right\|^{-2} X_{b}^{\varepsilon} \cdot \partial_{\theta}^{-1} u^{*}\right)\right]+\left(\mathfrak{P}_{b}^{\varepsilon}-\operatorname{Id}\right) \mathfrak{r i d i v} \mathfrak{v}_{b}^{\varepsilon}\left(\operatorname{div}\left(\Pi_{b}^{\varepsilon} u^{*}\right)\right) .
$$

In view of this relation, the point (ii) becomes clear.

Consider the Cauchy problem

$$
\mathfrak{d}_{0, \varepsilon} u^{*}+\varepsilon^{-1} \mathfrak{g r a d} \mathfrak{d}_{b}^{\varepsilon} p^{*}=f^{*}, \quad \mathfrak{d i v}_{b}^{\varepsilon} u^{*}=0, \quad u^{*}(0, .)=h^{*}(.)
$$

with data $f^{*} \in L_{T}^{2 *}$ and $h^{*} \in L^{2 *}$. Compose on the left with $\mathfrak{P}_{b}^{\varepsilon}$. It yields

$$
\mathfrak{d}_{0, \varepsilon} u^{*}=\mathfrak{P}_{b}^{\varepsilon} f^{*}, \quad u^{*}(0, .)=\mathfrak{P}_{b}^{\varepsilon} h^{*}(.) .
$$

The above Cauchy problem can be solved in two steps. First extract $u^{*}$ from the above equation. Then recover $p^{*}$ from the remaining relations.

Statement of the result. - A general principle is emphasized throughout the article [7]. Obvious instabilities can be absorbed by a dependent change of variables. But this manipulation induces a defect of hyperbolicity. Hidden instabilities can be implemented by soliciting this lack of hyperbolicity. On the contrary, they are not produced when this lack of hyperbolicity is compensated by some well adjusted parabolic perturbation $\mathcal{B}_{\mathfrak{q}}^{m}$.

This last observation is the source of the analysis which follows. From now on, we restrict to the case $\ell=2$ and $N \geq 3$. Thus, we are faced with strong oscillations. Note that the discussion is linked with the approach of [6] though the point of view is different.

Apply Proposition 6.1 with $b=(2, N)$. It furnishes a phase $\varphi_{b}^{\varepsilon} \equiv \varphi_{(2, N)}^{\varepsilon}$ which is henceforth fixed. It yields also some approximate solution

$$
u_{a}^{\varepsilon}(t, x, \theta):=u_{(2, N)}^{\varepsilon}(t, x, \theta), \quad p_{a}^{\varepsilon}(t, x, \theta):=p_{(2, N)}^{\varepsilon}(t, x, \theta)
$$

which is subjected to the system $(\mathcal{N S})$ with source term $f_{a}^{\varepsilon} \equiv f_{(2, N)}^{\varepsilon}$. To simplify the presentation, work again with the Laplacian $\Delta$. Look at

$$
\left\{\begin{array}{l}
\mathfrak{d}_{0, \varepsilon} u^{\varepsilon}+\left(u^{\varepsilon} \cdot \mathfrak{g r a d} \mathfrak{d}_{b}^{\varepsilon}\right) u^{\varepsilon}+\mathfrak{g r a d}_{b}^{\varepsilon} p^{\varepsilon}=\nu \varepsilon \Delta u^{\varepsilon}, \quad \mathfrak{d i v}_{b}^{\varepsilon} u^{\varepsilon}=0 \\
u^{\varepsilon}(0, x, \theta)=u_{a}^{\varepsilon}(0, x, \theta)
\end{array}\right.
$$

BULlEtin DE LA SOCIÉtÉ MATHÉMATIQUE DE FRANCE 
The Proposition 6.1 below is a small step in the direction of existence and convergence results.

TheOrem 6.1. - Fix $b=(2, N)$ with $N \geq 3$. Consider some approximate solution $\left\{u_{a}^{\varepsilon}\right\}_{\varepsilon}$ of $(\mathcal{N S})$. Then, there is $\left.\left.T>0, \varepsilon_{N} \in\right] 0,1\right]$ and $\nu_{N}>0$ such that for all $\left.\varepsilon \in] 0, \varepsilon_{N}\right]$ and for all $\nu>\nu_{N}$ the Cauchy problem (6.8) has a unique solution $\left(u^{\varepsilon}, p^{\varepsilon}\right)$ defined on the strip $[0, T] \times \mathbb{R}^{d} \times \mathbb{T}$. Moreover

$$
\left\{u^{\varepsilon}-u_{a}^{\varepsilon}\right\}_{\varepsilon}=O\left(\varepsilon^{\frac{1}{2}(N-3)}\right) .
$$

Note that the information (6.9) implies (6.7).

Proof of Theorem 6.1. - The system (6.8) amounts to the same thing as

$$
\left\{\begin{array}{c}
\partial_{t} \bar{u}^{\varepsilon}+\left(\bar{u}^{\varepsilon} \cdot \nabla\right) \bar{u}^{\varepsilon}+\operatorname{div}\left\langle u^{\varepsilon *} \otimes u^{\varepsilon *}\right\rangle+\nabla \bar{p}^{\varepsilon}=\nu \Delta_{x} \bar{u}^{\varepsilon} \\
\partial_{0, \varepsilon} u^{\varepsilon *}+\left(\bar{u}^{\varepsilon} \cdot \mathfrak{g r a d} \mathfrak{d}_{b}^{\varepsilon}\right) u^{\varepsilon *}+\varepsilon\left(u^{\varepsilon *} \cdot \nabla\right) \bar{u}^{\varepsilon} \\
\quad+\left[\left(u^{\varepsilon *} \cdot \mathfrak{g r a d} \mathfrak{d}_{b}^{\varepsilon}\right) u^{\varepsilon *}\right]^{*}+\mathfrak{g r a d} \mathfrak{d}_{b}^{\varepsilon} p^{\varepsilon *}=\nu \varepsilon \Delta u^{\varepsilon *} \\
\operatorname{div} \bar{u}^{\varepsilon}=\mathfrak{d i v} \mathfrak{v}_{b}^{\varepsilon} u^{\varepsilon *}=0 .
\end{array}\right.
$$

The equation (6.10) is also equivalent to solve the Cauchy problem

$$
\left\{\begin{array}{r}
P \partial_{t} \bar{u}^{\varepsilon}+P\left[\left(\bar{u}^{\varepsilon} \cdot \nabla\right) \bar{u}^{\varepsilon}\right]+P\left[\operatorname{div}\left\langle u^{\varepsilon *} \otimes u^{\varepsilon *}\right\rangle\right]=\nu \Delta_{x} \bar{u}^{\varepsilon}, \\
\mathfrak{P}_{b}^{\varepsilon} \partial_{0, \varepsilon} u^{\varepsilon *}+\mathfrak{P}_{b}^{\varepsilon}\left[\left(\bar{u}^{\varepsilon} \cdot \mathfrak{g r a d} \mathfrak{d}_{b}^{\varepsilon}\right) u^{\varepsilon *}\right]+\varepsilon \mathfrak{P}_{b}^{\varepsilon}\left[\left(u^{\varepsilon *} \cdot \nabla\right) \bar{u}^{\varepsilon}\right] \\
+\mathfrak{P}_{b}^{\varepsilon}\left[\left(u^{\varepsilon *} \cdot \mathfrak{g r a d} \mathfrak{d}_{b}^{\varepsilon}\right) u^{\varepsilon *}\right]^{*}=\nu \varepsilon \mathfrak{P}_{b}^{\varepsilon} \Delta u^{\varepsilon *},
\end{array}\right.
$$

associated with the compatible initial data

$$
\bar{u}^{\varepsilon}(0, .)=P \bar{u}_{b}^{\varepsilon}(0, .), \quad u^{\varepsilon *}(0, .)=\mathfrak{P}_{b}^{\varepsilon} u_{b}^{\varepsilon *}(0, .) .
$$

Obvious instabilities have an important consequence. To describe the related amplifications, it is necessary to introduce new quantities which correspond to the various phase shifts $\varphi_{j}$. In other words, the only way to get $L^{2}$-estimates is to blow up the state variables. This idea was exploited in [7] in the case of compressible Euler equations.

We use below a variant of this method.

Blow up. - Let $\iota \in \mathbb{R}_{+}$a parameter which will be adjusted later. Introduce the new unknown

$$
\begin{aligned}
d^{\varepsilon} & ={ }^{t}\left(\bar{d}^{\varepsilon}, d^{\varepsilon *}\right)={ }^{t}\left(P \bar{d}^{\varepsilon}, \mathfrak{P}_{b}^{\varepsilon} d^{\varepsilon *}\right) \\
& :=\varepsilon^{-\iota}\left(\varepsilon^{-\frac{1}{2}}\left(\bar{u}^{\varepsilon}-\bar{u}_{b}^{\varepsilon}\right),\left(u^{\varepsilon *}-u_{b}^{\varepsilon *}\right)\right), \quad b=(2, N) .
\end{aligned}
$$

Note that this transformation agrees with (5.10). The weight $\varepsilon^{-1 / \ell}$ in front of $\left(\bar{u}^{\varepsilon}-\bar{u}_{b}^{\varepsilon}\right)$ induces a shift on the indice $\ell$. Functions $\bar{U}_{\ell}$ and $U_{\ell-1}^{*}$ play now the same part related to the amplifications.

To write the equation on $d^{\varepsilon}$ in an abbreviated form, we need notations.

TOME $134-2006-\mathrm{N}^{\mathrm{O}} 1$ 
Quasilinear terms:

$$
\begin{aligned}
& \mathcal{L}_{11}^{\varepsilon} \bar{d}:=P\left[\left(\bar{u}_{b}^{\varepsilon} \cdot \nabla\right) \bar{d}\right], \\
& \mathcal{L}_{12}^{\varepsilon} d^{*}:=P\left[\operatorname{div}\left\langle\varepsilon^{-\frac{1}{2}} u_{b}^{\varepsilon *} \otimes d^{*}+d^{*} \otimes \varepsilon^{-\frac{1}{2}} u_{b}^{\varepsilon *}\right\rangle\right], \\
& \mathcal{L}_{21}^{\varepsilon} \bar{d}:=\varepsilon^{\frac{1}{2}} \mathfrak{P}_{b}^{\varepsilon}\left[\left(u_{b}^{\varepsilon *} \cdot \nabla\right) \bar{d}\right], \\
& \mathcal{L}_{22}^{\varepsilon} d^{*}:=\mathfrak{P}_{b}^{\varepsilon}\left[\left(\bar{u}_{b}^{\varepsilon} \cdot \nabla\right) d^{*}\right]+\varepsilon^{-1} \mathfrak{P}_{b}^{\varepsilon}\left[\left(u_{b}^{\varepsilon *} \cdot \mathfrak{g r a d} d_{b}^{\varepsilon}\right) d^{*}\right]^{*} \\
& \qquad \varepsilon^{-1} \mathfrak{P}_{b}^{\varepsilon}\left[\left(\partial_{t} \varphi_{b}^{\varepsilon}+\bar{u}_{b}^{\varepsilon} \cdot \nabla \varphi_{b}^{\varepsilon}\right) \partial_{\theta} d^{*}\right] .
\end{aligned}
$$

Semilinear terms:

$$
\begin{gathered}
A_{11}^{\varepsilon} \bar{d}:=P\left[(\bar{d} \cdot \nabla) \bar{u}_{b}^{\varepsilon}\right], \quad A_{21}^{\varepsilon} \bar{d}:=\mathfrak{P}_{b}^{\varepsilon}\left[\left(\bar{d} \cdot \mathfrak{g r a d} \mathfrak{d}_{b}^{\varepsilon}\right)\left(\varepsilon^{-\frac{1}{2}} u_{b}^{\varepsilon *}\right)\right], \\
A_{22}^{\varepsilon} d^{*}:=\mathfrak{P}_{b}^{\varepsilon}\left[\left(d^{*} \cdot \nabla\right) \bar{u}_{b}^{\varepsilon}\right]+\varepsilon^{-1} \mathfrak{P}_{b}^{\varepsilon}\left[\left(d^{*} \cdot \mathfrak{g r a d} \mathfrak{d}_{b}^{\varepsilon}\right) u_{b}^{\varepsilon *}\right]^{*} .
\end{gathered}
$$

Small quadratic terms:

$$
\begin{aligned}
& Q_{1}^{\varepsilon}:=\varepsilon^{\frac{3}{2}} P[\operatorname{div}(\bar{d} \otimes \bar{d})]+\varepsilon^{\frac{1}{2}} P\left[\operatorname{div}\left\langle d^{*} \otimes d^{*}\right\rangle\right], \\
& Q_{2}^{\varepsilon}:=\varepsilon^{\frac{1}{2}} \mathfrak{P}_{b}^{\varepsilon}\left[\left(\bar{d} \cdot \mathfrak{g r a d} \mathfrak{d}_{b}^{\varepsilon}\right) d^{*}\right]+\varepsilon^{\frac{3}{2}} \mathfrak{P}_{b}^{\varepsilon}\left[\left(d^{*} \cdot \nabla\right) \bar{d}\right]+\mathfrak{P}_{b}^{\varepsilon}\left[\left(d^{*} \cdot \mathfrak{g r a d} \mathfrak{d}_{b}^{\varepsilon}\right) d^{*}\right]^{*} .
\end{aligned}
$$

And error terms:

$$
e r_{1}^{\varepsilon}:=\varepsilon^{-\iota-\frac{1}{2}} P \bar{f}_{b}^{\varepsilon}, \quad e r_{2}^{\varepsilon}:=\varepsilon^{-\iota} \mathfrak{P}_{b}^{\varepsilon} f_{b}^{\varepsilon *} .
$$

With these conventions, the expression $d^{\varepsilon}$ is subjected to

$$
\left\{\begin{array}{c}
P \partial_{t} \bar{d}^{\varepsilon}+\mathcal{L}_{11}^{\varepsilon} \bar{d}^{\varepsilon}+\mathcal{L}_{12}^{\varepsilon} d^{\varepsilon *}+A_{11}^{\varepsilon} \bar{d}^{\varepsilon}+\varepsilon^{\iota-1} Q_{1}^{\varepsilon}+e r_{1}^{\varepsilon}=\nu P \Delta_{x} \bar{d}^{\varepsilon}, \\
\mathfrak{P}_{b}^{\varepsilon} \partial_{t} d^{\varepsilon *}+\mathcal{L}_{21}^{\varepsilon} \bar{d}^{\varepsilon}+\mathcal{L}_{22}^{\varepsilon} d^{\varepsilon *}+A_{21}^{\varepsilon} \bar{d}^{\varepsilon}+A_{22}^{\varepsilon} d^{\varepsilon *} \\
+\varepsilon^{\iota-1} Q_{2}^{\varepsilon}+e r_{2}^{\varepsilon}=\nu \mathfrak{P}_{b}^{\varepsilon} \Delta d^{\varepsilon *} .
\end{array}\right.
$$

Energy estimates are obtained at the level of (6.12). The related arguments which are classical. Below, we just sketch them.

$L^{2}$-estimates for the linear problem. - The linearized equations of Euler equations along the approximate solution $u_{b}^{\varepsilon}$ are obtained by removing $Q_{1}^{\varepsilon}$ and $Q_{2}^{\varepsilon}$ from (6.12). It yields a system which, at first sight, involves coefficients which are singular in $\varepsilon$. In fact, this is not the case. Let us explain why.

This is clear for $\mathcal{L}_{11}^{\varepsilon}, \mathcal{L}_{21}^{\varepsilon}$ and $A_{11}^{\varepsilon}$.

Since $u_{b}^{\varepsilon *}=O\left(\varepsilon^{1 / \ell}\right)$, this is also true for $\mathcal{L}_{12}^{\varepsilon}$ and $A_{21}^{\varepsilon}$.

The contributions which in $\mathcal{L}_{22}^{\varepsilon}$ have $\varepsilon^{-1}$ in factor give no trouble since

$$
\partial_{t} \varphi_{b}^{\varepsilon}+\bar{u}_{b}^{\varepsilon} \cdot \nabla \varphi_{b}^{\varepsilon}=O\left(\varepsilon^{\frac{1}{2} N}\right)=O(\varepsilon), \quad u_{b}^{\varepsilon *} \cdot \nabla \varphi_{b}^{\varepsilon}=v_{b}^{\varepsilon *}=O\left(\varepsilon^{1+1 / \ell}\right) .
$$

Now, look at $A_{22}^{\varepsilon}$. Recall that $d^{\varepsilon *}=\mathfrak{P}_{b}^{\varepsilon} d^{\varepsilon *}$ which means that

$$
\varepsilon^{-1} \partial_{\theta} d^{\varepsilon *} \cdot \nabla \varphi_{b}^{\varepsilon}=-\operatorname{div} d^{\varepsilon *} .
$$

Therefore

$$
\varepsilon^{-1}\left[\left(d^{\varepsilon *} \cdot \mathfrak{g r a d} \mathfrak{d}_{b}^{\varepsilon}\right) u_{b}^{\varepsilon *}\right]^{*}=\left[\left(d^{\varepsilon *} \cdot \nabla\right) u_{b}^{\varepsilon *}-\operatorname{div} \partial_{\theta}^{-1} d^{\varepsilon *} \partial_{\theta} u_{b}^{\varepsilon *}\right]^{*}
$$

which is obviously an operator of the order one.

BULletin DE LA SOCIÉtÉ MATHÉMATIQUE DE FRANCE 
Observe that these manipulations and the blow up procedure induce a loss of hyperbolicity. In other words, the system (6.12) involve operators which are non singular with respect to $\varepsilon$, which are all of the order one, but which are not all skew-symmetric. When $\nu=0$, this is the source of hidden instabilities. When $\nu \geq \nu_{N}>0$ with $\nu_{N}$ large enough, the losses of derivatives can be compensated by the viscosity. This is the key to $L^{2}$-estimates.

The non linear problem and higher order estimates. - Let $\sigma$ be the smaller integer such that $\sigma \geq \frac{1}{2}(d+3)$. If the life span $T_{\varepsilon}$ of the exact solution $u^{\varepsilon}$ is finite, we must have

$$
\lim _{t \rightarrow T_{\varepsilon}}\left\|u^{\varepsilon}(t, .)\right\|_{H^{\sigma}}=+\infty .
$$

Thus, Theorem 6.1 is a consequence of the following majoration

$$
\sup \left\{\left\|u^{\varepsilon}(t, .)\right\|_{H^{\sigma}} ; t \in\left[0, \min \left(T_{\varepsilon}, T\right)\right]\right\} \leq C<\infty .
$$

Consider the set

$$
\mathcal{Z}_{\varepsilon}:=\left\{\mathfrak{d}_{0, \varepsilon}, \ldots, \mathfrak{d}_{d, \varepsilon}, \partial_{\theta}\right\} .
$$

Observe that the commutator of two vector fields in $\mathcal{Z}^{\varepsilon}$ is a linear combination of elements of $\mathcal{Z}^{\varepsilon}$ with coefficients in $C^{\infty}$. Build the differential operators

$$
\mathcal{Z}_{\varepsilon}^{k}:=\mathcal{Z}_{1} \circ \cdots \circ \mathcal{Z}_{k}, \quad \mathcal{Z}_{j} \in \mathcal{Z}_{\varepsilon}, \quad k \leq \sigma .
$$

It suffices to show that

$$
\max _{0 \leq k \leq \sigma} \sup \left\{\left\|\varepsilon^{-k} \mathcal{Z}_{\varepsilon}^{k} u^{\varepsilon}(t, .)\right\|_{L^{2}} ; t \in\left[0, \min \left(T_{\varepsilon}, T\right)\right]\right\} \leq C<\infty .
$$

Pick some $\mathcal{Z}_{\varepsilon}^{k}$ with $k \leq \sigma$. Apply $\mathcal{Z}_{\varepsilon}^{k}$ on the left of (6.12). Use the point (i) of Lemma 6.2 to pass through $\mathfrak{P}_{b}^{\varepsilon}$. We get an equation on $\mathcal{Z}_{\varepsilon}^{k} d^{\varepsilon *}$.

Perform $L^{2}$-estimates on this equation, as in the preceding paragraph. Take $\iota=1$ which implies that $Q_{1}^{\varepsilon}$ and $Q_{2}^{\varepsilon}$ have $\varepsilon^{0}$ in factor. Therefore, the contributions due to $Q_{1}^{\varepsilon}$ and $Q_{2}^{\varepsilon}$ can be controled by way of the a priori estimate and the viscosity. Since

$$
e r_{1}^{\varepsilon}=O\left(\varepsilon^{\frac{1}{2}(N-5)}\right), \quad e r_{2}^{\varepsilon}=O\left(\varepsilon^{\frac{1}{2} N-2}\right), \quad d^{\varepsilon}(0, .) \equiv 0,
$$

we find $d^{\varepsilon}=O\left(\varepsilon^{\frac{1}{2}(N-5)}\right)$ which leads to $u^{\varepsilon}-u_{a}^{\varepsilon}=O\left(\varepsilon^{\frac{1}{2}(N-3)}\right)$.

Note that the size $\varepsilon^{\frac{1}{2} N-1}$ of the source term $f_{b}^{\varepsilon}$ is turned into $\varepsilon^{\frac{1}{2}(N-3)}$. This (fixed) lost of the weight $\varepsilon^{\frac{1}{2}}$ corresponds exactly to what is listed at the level of obvious instabilities.

\section{BIBLIOGRAPHY}

[1] Bardos (C.) - What use for the mathematical theory of the Navier-Stokes equations, in Mathematical Fluid Mechanics, Adv. Math. Fluid Mech., Birkhäuser, Basel, 2001, pp. 1-25.

TOME $134-2006-\mathrm{N}^{\mathrm{O}} 1$ 
[2] Beale (J.T.), Kato (T.) \& MAJdA (A.) - Remarks on the breakdown of smooth solutions for the 3D Euler equations, Comm. Math. Phys. (1994), pp. 61-66.

[3] Bertozzi (A.) \& Majda (A.) - Vorticity and Incompressible Flow, Cambridge University Press, 2002.

[4] Chacon Rebollo (T.) - Oscillations due to the transport of microstructures, SIAM J. Appl. Math., t. 48 (1988).

[5] Chemin (J.-Y.) - Perfect Incompressible Fluids, Oxford Lecture Series in Math. and its Applications, vol. 14, The Clarendon Press, Oxford University Press, New York, 1998, translated from the 1995 French original by Isabelle Gallagher and Dragos Iftimie.

[6] Chemin (J.-Y.), Desjardins (B.), Gallagher (I.) \& Grenier (E.) - Fluids with anisotropic viscosity, Math. Modelling Num. Anal., t. 34 (2000), pp. 315-335.

[7] Cheverry (C.) - Propagation of oscillations in real vanishing viscosity limit, Comm. Math. Phys., t. 247 (2004), pp. 655-695.

[8] Cheverry (C.), Guès (O.) \& Métivier (G.) - Oscillations fortes sur un champ linéairement dégénéré, Ann. Sci. École Norm. Sup. (4), t. 36 (2003), pp. 691-745.

[9] _ Large amplitude high frequency waves for quasilinear hyperbolic systems, Advances in Differential Equations (to appear).

[10] Constantin (P.) \& Fefferman (C.) - Direction of vorticity and the problem of global regularity for the Navier-Stokes equations, Indiana Univ. Math. J., t. 42 (1993), pp. 775-789.

[11] Corli (A.) \& GuÈs (O.) - Stratified solutions for systems of conservation laws, Trans. Amer. Math. Soc., t. 353 (2001), pp. 2459-2486.

[12] DiPerna (R.-J.) \& MAJdA (A.-J.) - Oscillations and concentrations in weak solutions of the incompressible fluid equations, Comm. Math. Phys., t. 108 (1987), pp. 667-689.

[13] Duchon (J.) \& Robert (R.) - Inertial energy dissipation for weak solutions of incompressible Euler and Navier-Stokes equations, Nonlinearity, t. 13 (2000), pp. 249-255.

[14] E. (WEINAN) - Homogenization of linear and non linear transport equations, Comm. Math. Phys., t. 45 (1992), pp. 301-326.

[15] , Propagation of oscillations in the solutions of $1 D$ compressible fluid equations, Comm. Partial Differential Equations, t. 17 (1992), pp. 347-370.

[16] Foias (C.), Manley (P.-P.), Rosa (R.) \& Temam (R.) - Cascade of energy in turbulent flows, C. R. Acad. Sci. Paris Sér. I Math., t. 332 (2001), pp. 509-514.

[17] Friedlandler (S.), Strauss (W.) \& Vishik (M.) - Nonlinear instability in an ideal fluid, , t. 14 (1997), pp. 187-209. 
[18] GÉrard (P.) - Microlocal defect measures, Comm. Partial Differential Equations, t. 16 (1991), pp. 1761-1794.

[19] Grenier (E.) - On the nonlinear instability of Euler and Prandtl equations, Comm. Pure Appl. Math., t. 53 (2000), pp. 1067-1091.

[20] Gù̀s (O.) - Ondes multidimensionnelles $\varepsilon$-stratifiées et oscillations, Duke Math. J., t. 68 (1992), pp. 401-446.

[21] _ Développement asymptotique de solutions exactes de systèmes hyperboliques quasilinéaires, Asymp. Anal., t. 6 (1993), pp. 241-269.

[22] Joly (J.-L.), MÉtivier (G.) \& Rauch (J.) - Transparent non linear geometric optics and Maxwell-Bloch equations, J. Differential Equations, t. 166 (2000), pp. 175-250.

[23] LANNEs (D.) - Secular growth for symmetric hyperbolic systems, J. Differential Equations, t. 90 (2003), pp. 466-503.

[24] Lesieur (M.) - Turbulence in Fluids, Fluid Mechanics and its Applications, Kluwer Academic Publishers Group, Dordrecht, 1997.

[25] Lions (P.-L.) - Mathematical Topics in Fluid Mechanics, Vol. 1, Incompressible Models, Oxford Science Publications.

[26] Mclaughlin (D.W.), Papanicolaou (G.C.) \& Pironneau (O.R.) Convection of microstructure and related problems, SIAM J. Appl. Math., t. 45 (1985).

[27] Schochet (S.) - Fast singular limits of hyperbolic PDEs, J. Differential Equations, t. 114 (1994), pp. 476-512.

[28] SERRE (D.) - Oscillations nonlinéaires de haute fréquence, dim $\geq 2$, in Nonlinear Variational Problems and Partial Differential Equations (Marino (A.) \& Murthy (M.K.V.), eds.), Pitman Res. Notes in Math., vol. 320, Longman, London, 1995, pp. 245-294.

[29] Sideris (T.) - Formation of singularities in compressible fluids, Commun. Math. Phys., t. 101 (1985), pp. 475-485. 